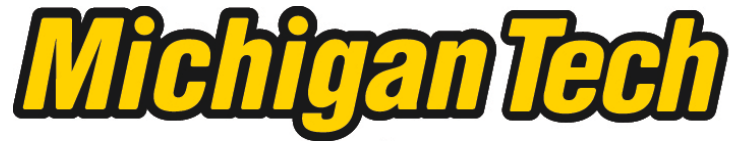 \\ Michigan Technological University Create the Future Digital Commons @ Michigan Tech
}

2012

\section{Engineering design motivations : a qualitative and quantitative analysis of university students}

Kristine Louise Guzak

Michigan Technological University

Follow this and additional works at: https://digitalcommons.mtu.edu/etds

Part of the Civil and Environmental Engineering Commons

Copyright 2012 Kristine Louise Guzak

\section{Recommended Citation}

Guzak, Kristine Louise, "Engineering design motivations : a qualitative and quantitative analysis of university students ", Master's Thesis, Michigan Technological University, 2012.

https://doi.org/10.37099/mtu.dc.etds/242

Follow this and additional works at: https://digitalcommons.mtu.edu/etds

Part of the Civil and Environmental Engineering Commons 
ENGINEERING DESIGN MOTIVATIONS: A QUALITATIVE AND QUANTITATIVE ANALYSIS OF UNIVERSITY STUDENTS

By

Kristine Louise Guzak

A THESIS

Submitted in partial fulfillment of the requirements for the degree of

MASTER OF SCIENCE

(Environmental Engineering)

MICHIGAN TECHNOLOGICAL UNIVERSITY

2012

Copyright: Kristine L. Guzak 2012 

This thesis, "Engineering Design Motivations: A Qualitative and Quantitative Analysis of University Students," is hereby approved in partial fulfillment of the requirements for the Degree of MASTER OF SCIENCE IN ENVIRONMENTAL ENGINEERING

Department of Civil and Environmental Engineering

Signatures:

Thesis Advisor

Dr. Kurt Paterson

Committee Member

Dr. Bill Kennedy

Committee Member

Dr. Mark Rouleau

Department Chair

Dr. David Hand

Date 

For my family and friends, who have been my motivators 



\section{Table of Contents}

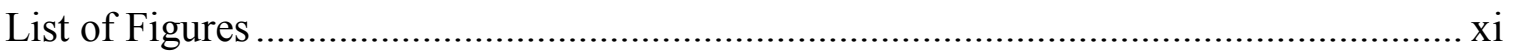

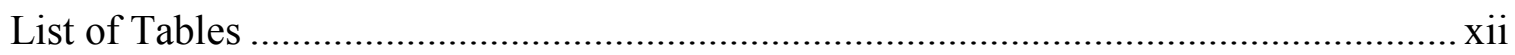

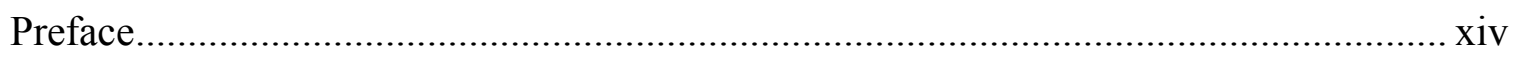

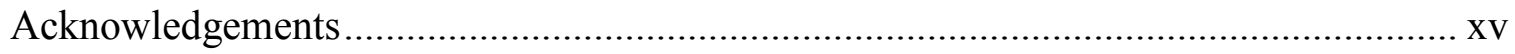

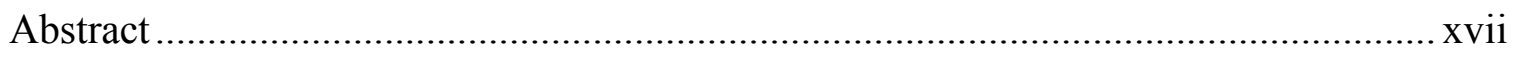

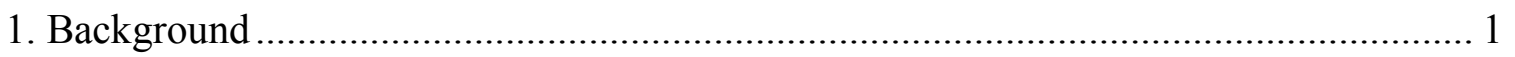

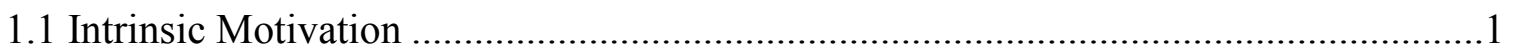

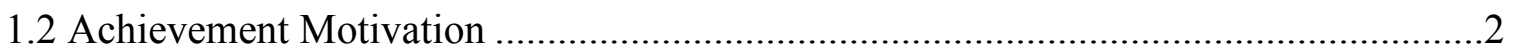

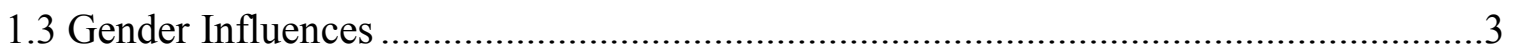

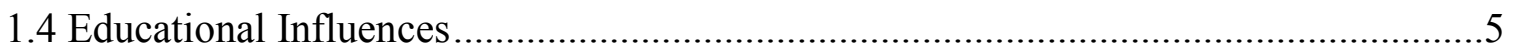

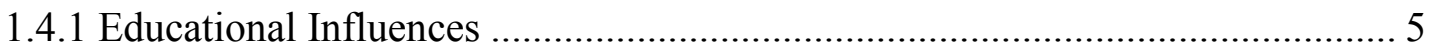

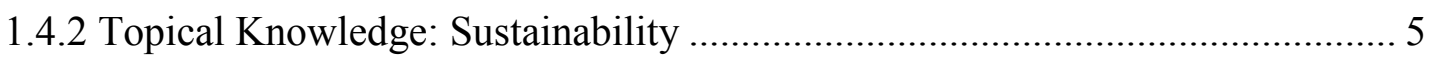

1.4.3 Character Development: Cultural Awareness ................................................. 6

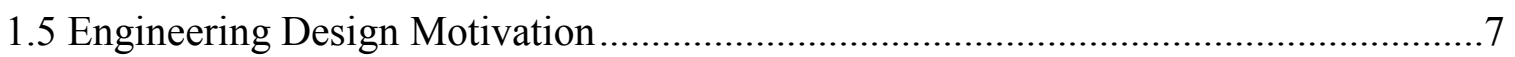

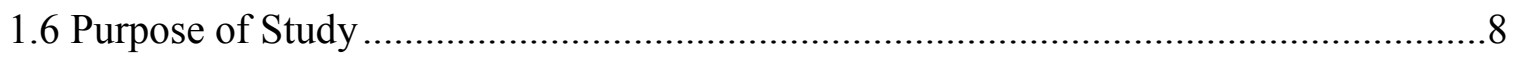

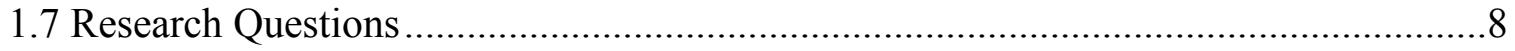

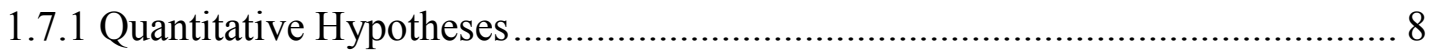

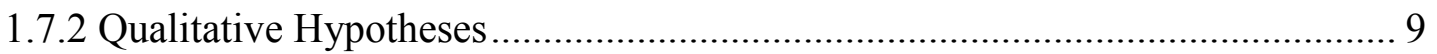

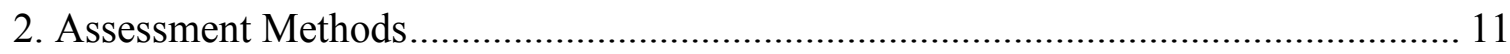

2.1 Quantitative Methodology: Assessment Instruments .................................................12

2.1.1 Readiness to Serve: Readiness Inventory ................................................. 12

2.1.2 Intercultural Awareness: Intercultural Development Inventory ....................... 12

2.1.3 Knowledge, Skills, and Attitudes: Impacts of Service on Engineering Students

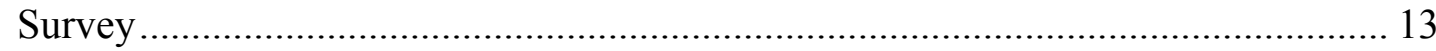

2.1.4 Sustainable Engineering Knowledge: SESL ……………............................. 14

2.1.5 Motivations Narratives: Frequency Analysis .................................................. 14

2.1.6 Interview Protocol: Frequency Analysis .................................................... 14

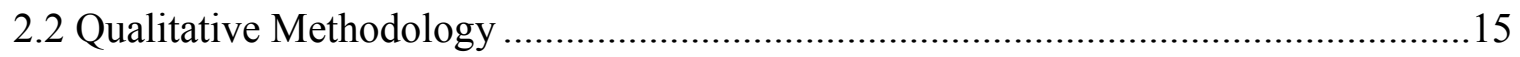

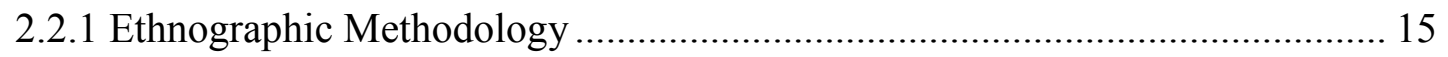

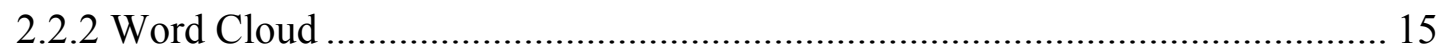

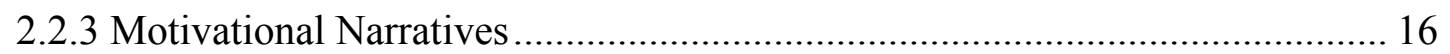

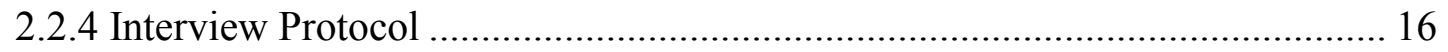




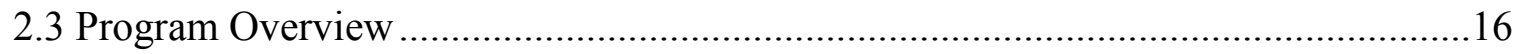

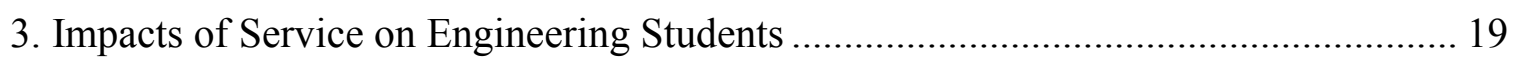

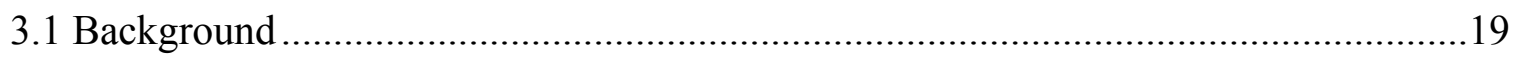

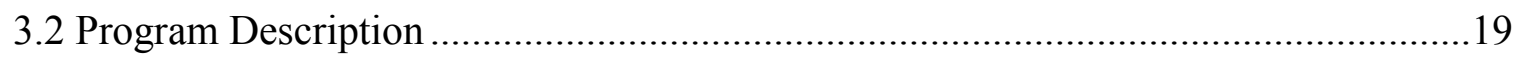

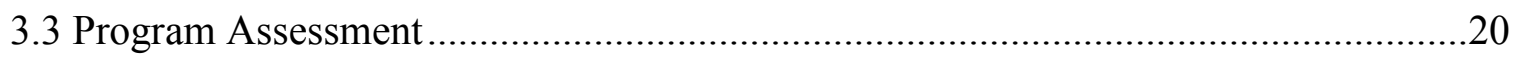

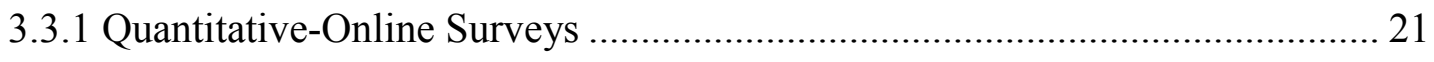

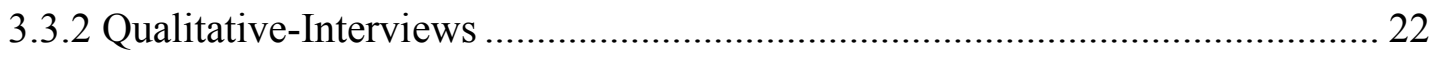

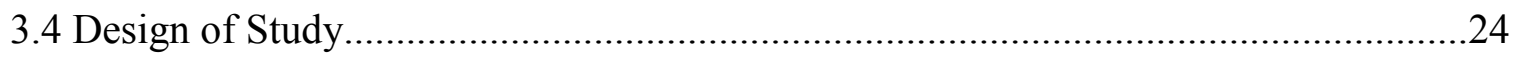

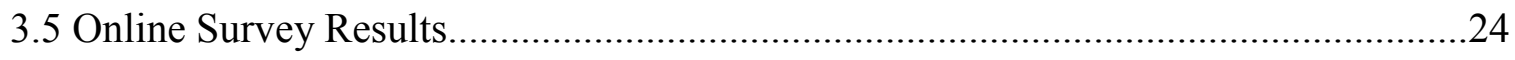

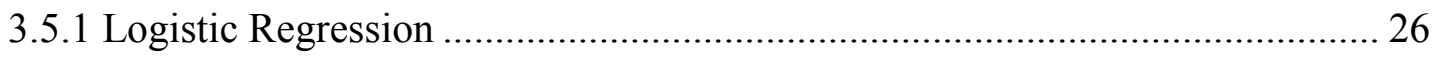

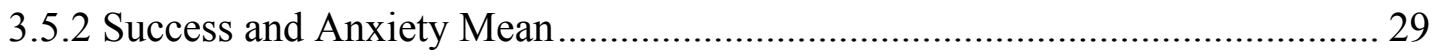

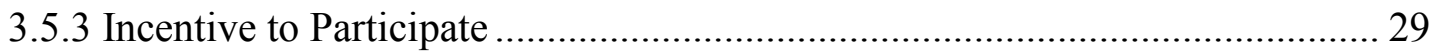

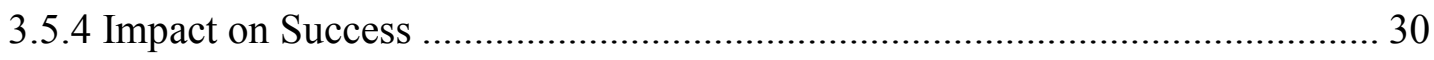

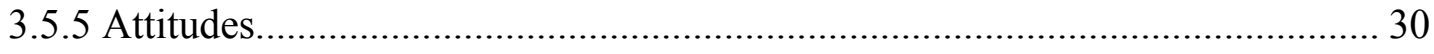

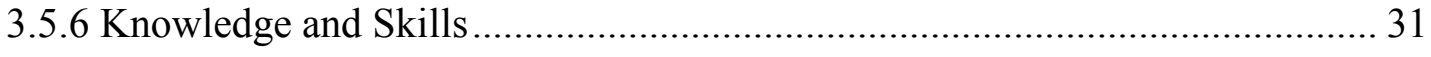

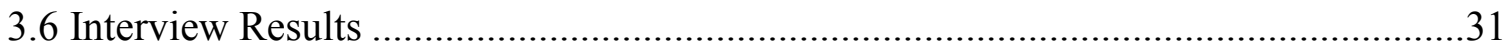

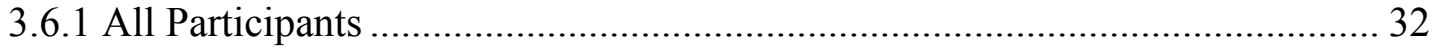

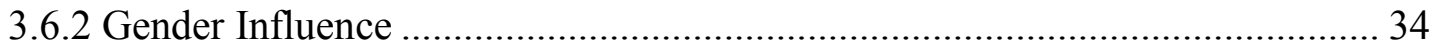

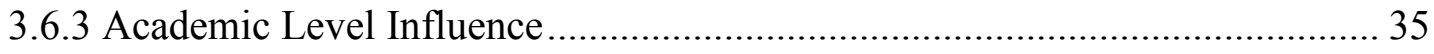

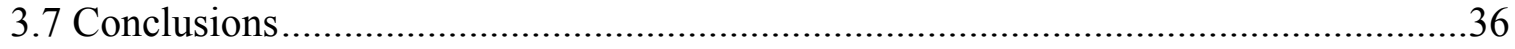

3.8 Next Steps

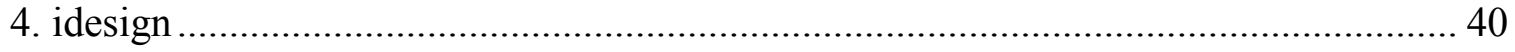

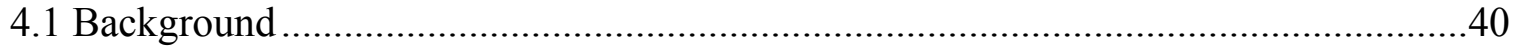

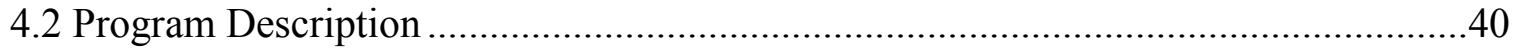

4.3 Program Assessment ....................................................................................... 40

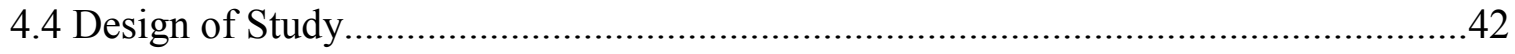

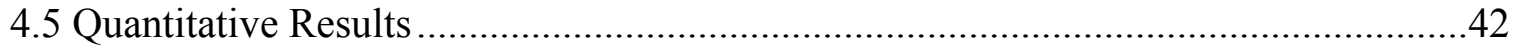

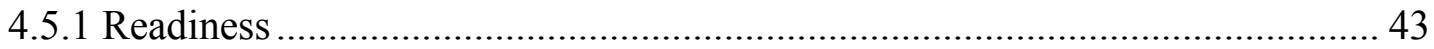

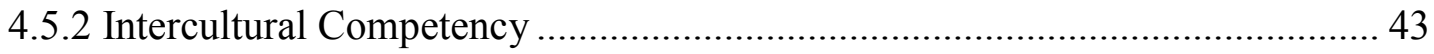

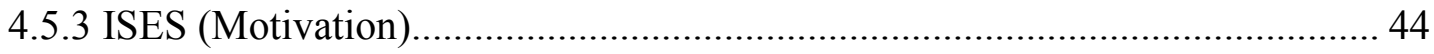

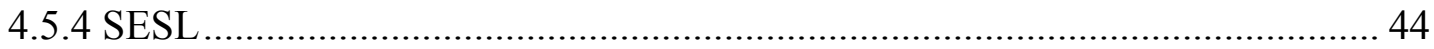

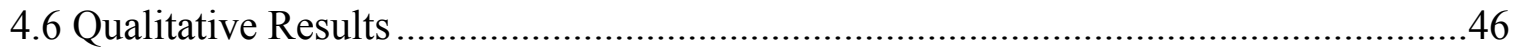

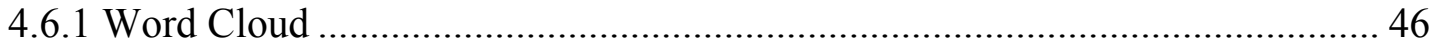

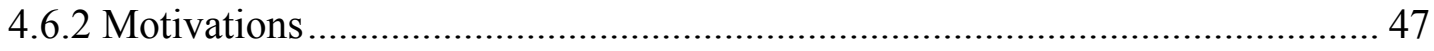




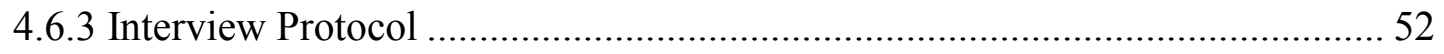

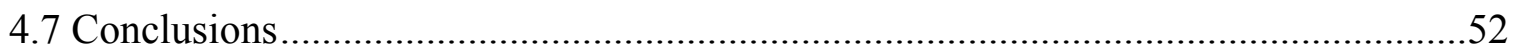

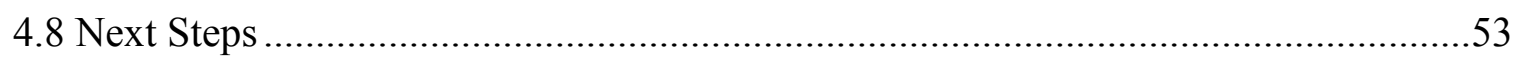

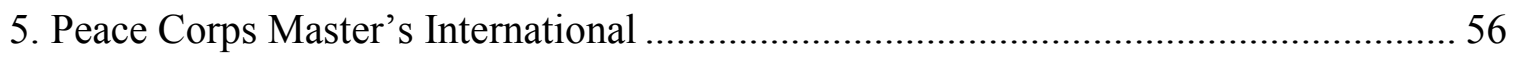

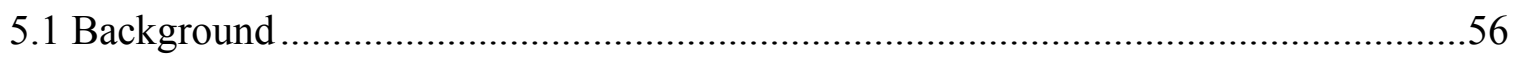

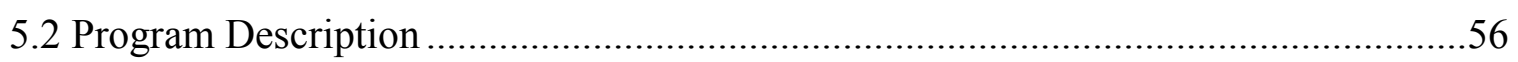

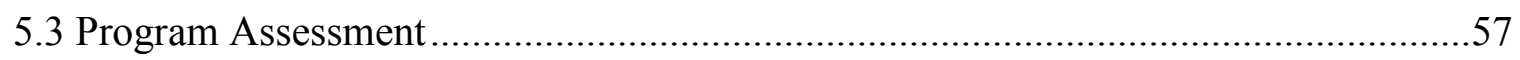

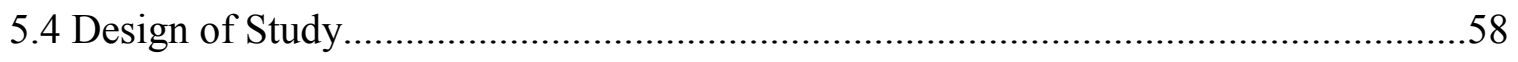

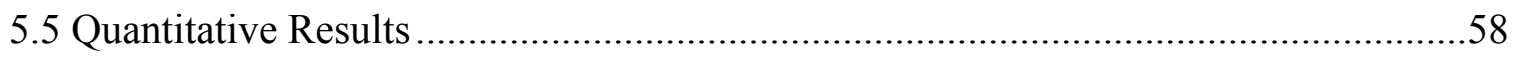

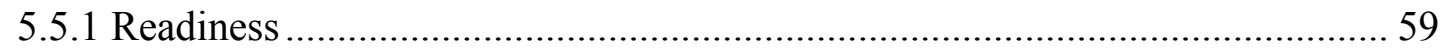

5.5.2 Intercultural Competency ……………..................................................... 59

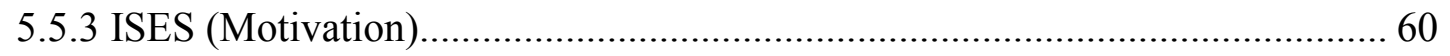

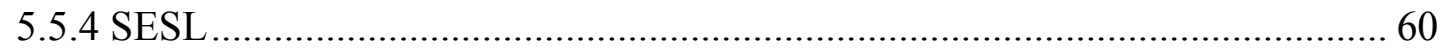

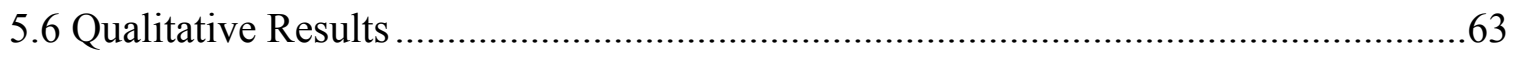

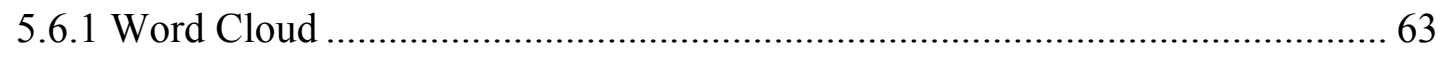

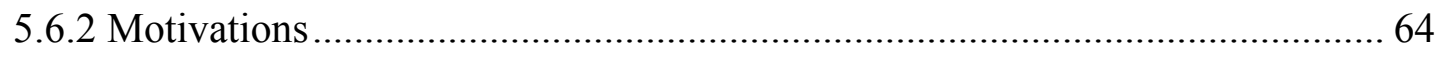

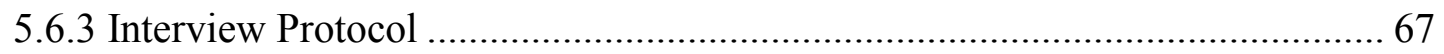

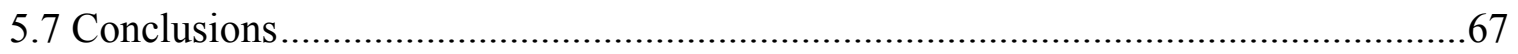

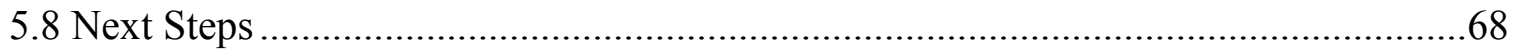

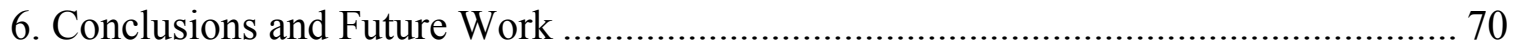

6.1 Quantitative Methods Conclusions ........................................................................

6.1.1 ISES Quantitative Conclusions …………………...................................... 70

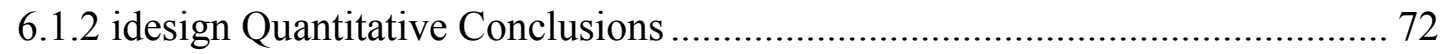

6.1.3 PCMI Quantitative Conclusions .................................................................. 73

6.2 Qualitative Methods Conclusions …………………............................................74

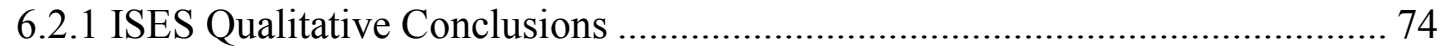

6.2.2 idesign Qualitative Conclusions ................................................................. 75

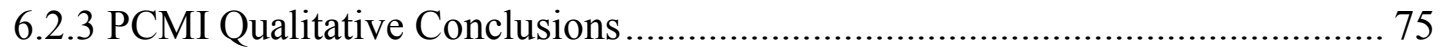

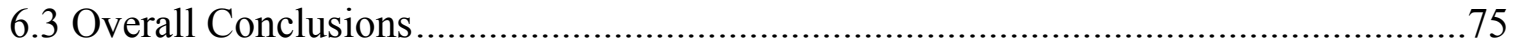

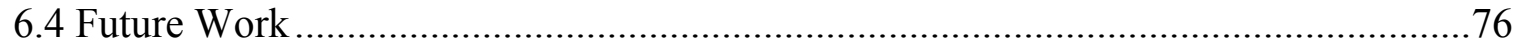

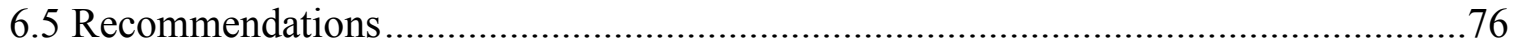

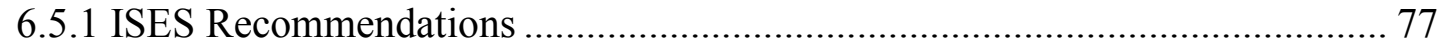

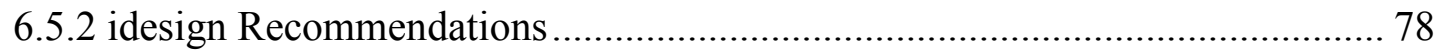

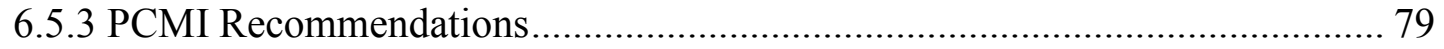




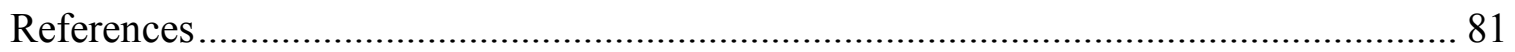

7. Appendix: Quantitative Assessment Instrument Samples ......................................... 87

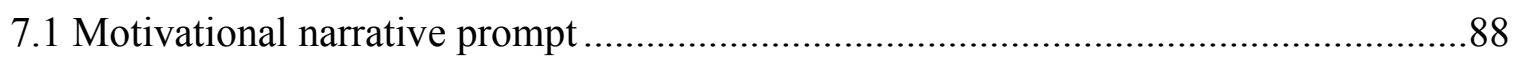

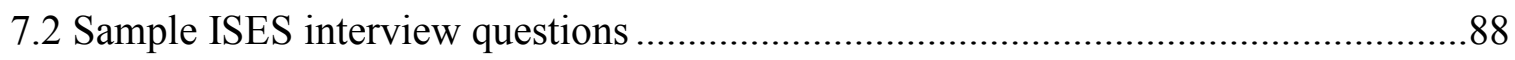

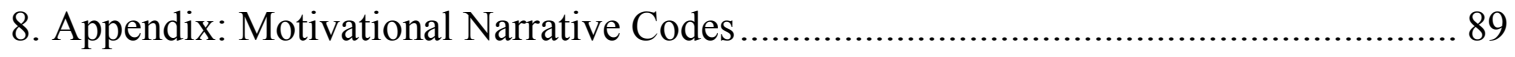

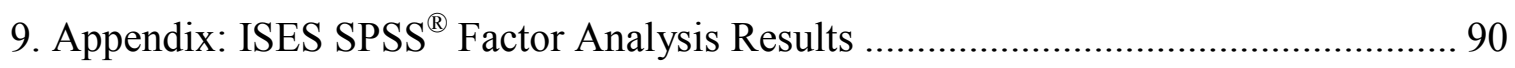

9.1 Knowledge and Skills Part 1 Factor Analysis...........................................................

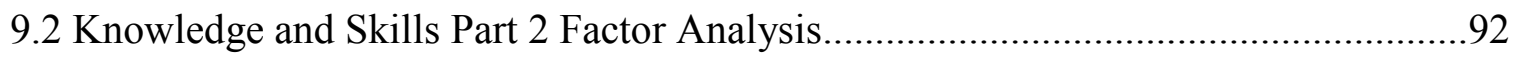

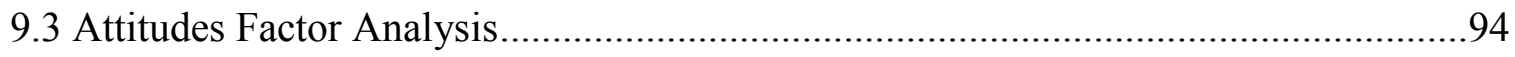

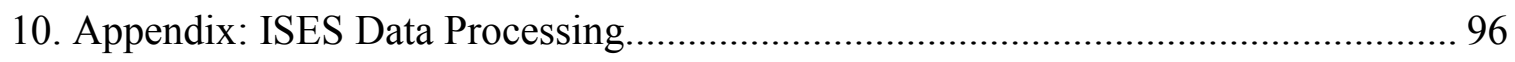

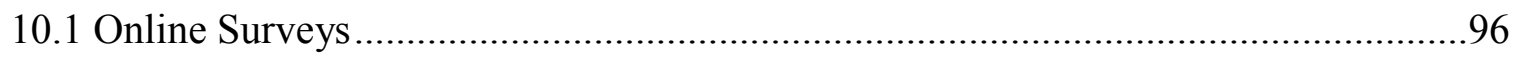

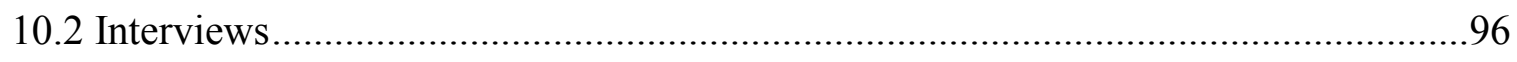

11. Appendix: Logistic Regression Results and Tables............................................... 98

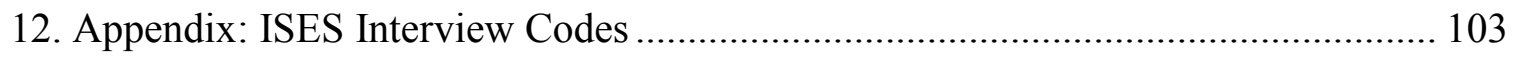

13. Appendix: Copyright Permission Form Documentation for Portions of Chapters 4 and

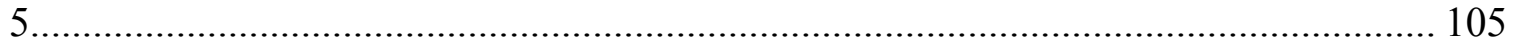




\section{List of Figures}

Figure 2.1: Intercultural Development Inventory (IDI) spectrum 13

Figure 3.1: Spring 2011 to Spring 2012 motivation score distribution within H-S (HighStatic), H-D (High-Decreasing), L-I (Low-Increasing), and L-S (Low-Static)

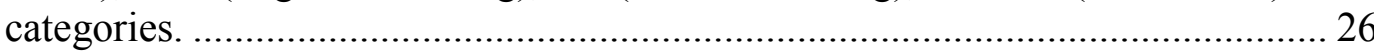

Figure 3.2: Spring 2011 average motivation score distribution by gender........................ 35

Figure 4.1: idesign student participant demographics in two categories: gender, and prior international experience $(\mathrm{n}=36)$.................................................................... 42

Figure 4.2: Word cloud of motivation codes for idesign motivational narratives $(n=36) 47$

Figure 4.3: International experience for (left to right): all idesign women $(\mathrm{n}=23)$ and all men $(\mathrm{n}=13)$. Low is 0 to 10 weeks, medium is 10 to 30 weeks, and high is greater than 30 weeks of living and traveling internationally.......................................... 48

Figure 4.4: International experience for (left to right): all undergraduates $(\mathrm{n}=36)$ and all graduate students $(\mathrm{n}=19)$. Low is 0 to 10 weeks, medium is 10 to 30 weeks, and high is greater than 30 weeks of living and traveling internationally ....................50

Figure 5.1: PCMI Student participant demographics in two categories: gender, and prior

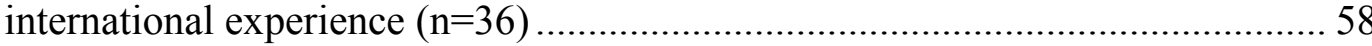

Figure 5.2: Word cloud of motivation codes for PCMI motivational narratives $(\mathrm{n}=19)$.. 63

Figure 5.3: International experience for (left to right): all women $(\mathrm{n}=10)$ and all men $(\mathrm{n}=9)$. Low is 0 to 10 weeks, medium is 10 to 30 weeks, and high is greater than 30 weeks of living and traveling internationally

Figure 7.1: Readiness assessment inventory (Jesiek et al. 2011) .................................... 87

Figure 7.2: ISES survey sample questions (Pierrakos et al. 2008a; Pierrakos et al. 2008b; Pierrakos et al. 2007)

Figure 7.3: SESL survey sample questions (McCormick et al. 2010; McCormick et al. 2011) 


\section{List of Tables}

Table 2.1 List of Assessment instruments ................................................................. 11

Table 3.1 Assessment instruments use in the Impacts of Service on Engineering Students

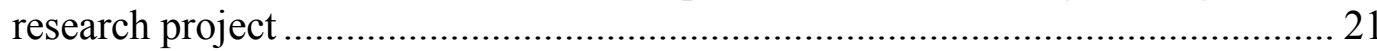

Table 3.2 Three-semester question breakdown for the Impacts of Service on Engineering

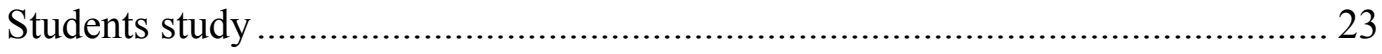

Table 3.3 Interview LTS experience demographics …………………….................. 24

Table 3.4 Interview "extreme” demographics ................................................................. 26

Table 3.5 Logistic regression significant independent values table ................................ 28

Table 3.6 Top five motivations expressed by all "Extreme" interview participants $(\mathrm{n}=120)$; rank (frequency), $\mathrm{n}=1035$ code tags from all participants ...................... 33

Table 3.7 Top five motivations expressed by men and women ( $\mathrm{n}=54$ and 36 , respectively) "Extreme" interview participants; rank (frequency), n=572 and 463, respectively, code tags for all three semesters ................................................................... 35

Table 3.8 Top five motivations expressed by freshmen and juniors ( $\mathrm{n}=51$ and 69 , respectively) "Extreme" interview participants; rank (frequency), n=442 and 593, respectively, code for all three semesters ........................................................... 36

Table 4.1 idesign assessment instruments .................................................................. 41

Table 4.2 idesign assessment instruments results summary ........................................... 45

Table 4.3 idesign Readiness and Intercultural Development Inventory results summary

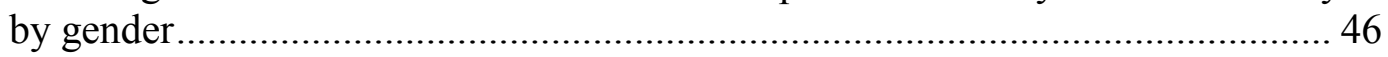

Table 4.4 idesign Motivation and SESL results summary by gender............................. 46

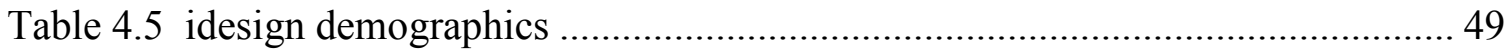

Table 4.6 Top five motivations expressed by idesign men and women; rank (frequency), $\mathrm{n}=214$ code tags within 36 student essays .......................................................... 49

Table 4.7 Top five motivations expressed by undergraduate and graduate students; rank (frequency), $\mathrm{n}=332$ tags within 55 student essays .............................................. 50

Table 4.8 Top three motivations expressed by idesign and PCMI students with low, medium, and high international experience; rank (frequency), $\mathrm{n}=332$ code tags within 55 student essays............................................................................... 52

Table 5.1 PCMI assessment instruments ................................................................ 57

Table 5.2 PCMI Readiness and IDI assessment instruments results summary ............... 61

Table 5.3 PCMI Motivation and SESL assessment instruments results summary .......... 61

Table 5.4 PCMI Readiness assessment instruments results summary by gender............ 62

Table 5.5 PCMI IDI assessment instruments results summary by gender ...................... 62 
Table 5.6 PCMI Motivation assessment instruments results summary by gender 62

Table 5.7 PCMI SESL assessment instruments results summary by gender.................. 63

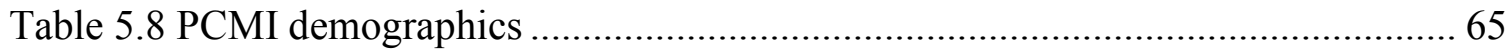

Table 5.9 Top five motivations expressed by men and women; rank (frequency), $n=118$

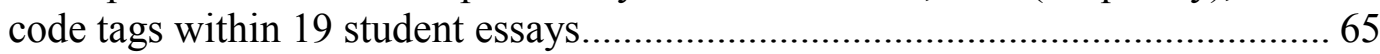

Table 5.10 Top motivation essay codes as response to reasons for participation in an international service program $(n=333$ code tags for 55 student essays $)$.............. 66

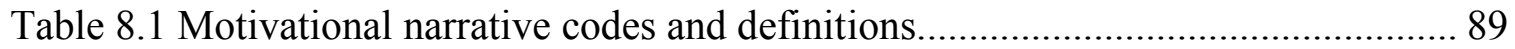

Table 9.1 Knowledge and skills part 1 factor analysis rotated component matrix .......... 90

Table 9.2 Knowledge and skills part 1 factor analysis total variance explained ............. 91

Table 9.3 Knowledge and skills part 2 factor analysis rotated component matrix .......... 92

Table 9.4 Knowledge and skills part 2 factor analysis total variance explained ............. 93

Table 9.5 Attitudes factor analysis rotated component matrix .................................... 94

Table 9.6 Attitudes factor analysis total variance explained ..................................... 95

Table 11.1 Logistic regression with High-Static as a reference category with grey highlighting the significant independent variables ........................................... 98

Table 11.2 Continuation of Logistic regression with High-Static as a reference category with grey highlighting the significant independent variables ............................ 99

Table 11.3 Logistic regression with Low-Static as a reference category with grey highlighting the significant independent variables 100

Table 11.4 Continuation of Logistic regression with Low-Static as a reference category with grey highlighting the significant independent variables.....

Table 11.5 Continuation of Logistic regression with Low-Static as a reference category with grey highlighting the significant independent variables ........................... 102

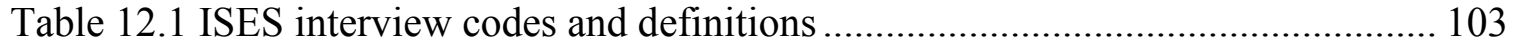

Table 12.2 Continuation of ISES interview codes and definitions ............................... 104 


\section{Preface}

Components of two conference papers were utilized in the creation of this thesis. Neither paper was used in its entirety. Additionally, any tables or figures that appear in this thesis from these papers were recreated with additional information. The use of these papers is further detailed below:

1. Guzak K, Paterson KG. 2011.Learning Through Service Student Motivations. ASEE North Midwest Conference.

2. Guzak K, Paterson KG, Archer A. 2012. A Comparative Assessment of Graduate versus Undergraduate Student Outcomes via International Community Engagement Programs. ASEE Annual Conference.

Both of these papers were co-authored by my advisor, Kurt Paterson, and myself with additional assistance by another Environmental Engineering Master's student for the second. My contribution consisted of, but isn't limited to, data collation and analysis, structuring of both documents, and presenting of papers. Chapters 2 through 5 utilized some of the content of both of these documents.

Additionally, this project includes human subject research, which has been approved by the institutional review board (IRB). Projects were approved under M0322, M0901 and M0621 project numbers. 


\section{Acknowledgements}

First of all, I would like to thank my advisor Kurt Paterson for his help and support throughout my career at Michigan Tech. I appreciate all of the unyielding encouragement and advice he gave me; he helped me realize my potential. This research could not have been conducted without the help of several colleagues and friends, who supplied ideas, help, and encouragement throughout the process. Thanks goes to my research colleague Chris Swan, my committee members Mark and Bill, my interview transcribers and friends Allie Archer, Alex Wohlgemuth, Olga Cano, Patrick Spenser, and everyone else who helped me with the details along the way. This project could not have been possible without the generous funding by the National Science Foundation. Their financial assistance is much appreciated.

Finally, I would like to thank my family and friends for their love and support throughout my education. I am especially grateful to my parents, sister, and brother who have always supported and encouraged me in all of my endeavors. 


\begin{abstract}
What motivates students to perform and pursue engineering design tasks? This study examines this question by way of three Learning Through Service (LTS) programs: 1) an on-going longitudinal study examining the impacts of service on engineering students, 2 ) an on-going analysis of an international senior design capstone program, and 3) an ongoing evaluation of an international graduate-level research program. The evaluation of these programs incorporates both qualitative and quantitative methods, utilizing surveys, questionnaires, and interviews, which help to provide insight on what motivates students to do engineering design work. The quantitative methods were utilized in analyzing various instruments including: a Readiness assessment inventory, Intercultural Development Inventory, Sustainable Engineering through Service Learning survey, the Impacts of Service on Engineering Students' survey, Motivational narratives, as well as some analysis for interview text. The results of these instruments help to provide some much needed insight on how prepared students are to participate in engineering programs. Additional qualitative methods include: Word clouds, Motivational narratives, as well as interview analysis. This thesis focused on how these instruments help to determine what motivates engineering students to pursue engineering design tasks. These instruments aim to collect some more in-depth information than the quantitative instruments will allow. Preliminary results suggest that of the 120 interviews analyzed Interest/Enjoyment, Application of knowledge and skills, as well as gaining knowledge are key motivating factors regardless of gender or academic level. Together these findings begin to shed light on what motivates students to perform engineering design tasks, which can be applied for better recruitment and retention in university programs.
\end{abstract}


xviii 


\section{Background}

Over the last few years, concerns have escalated among many national organizations that technical expertise is no longer solely sufficient for the development of future engineers (Boyer 1996; Grandin and Hirleman 2009; Katehi 2005; Sigma Xi 2007; Vest 2006). Additionally, in the United States, engineering programs continue to struggle to attract students, especially women and minorities, despite decades of strategies to change these patterns (Jordan et al. 2011; National Science Foundation 2011; Seymour and Hewitt 2000; Tinto 1994). Independent of these challenges, students have rapidly created extracurricular service efforts. Of considerable note is the quick emergence of Engineers Without Borders chapters at more than 200 universities within eight years (Paterson et al. 2010). In some institutions, this service involvement has fueled the creation of courses and programs that offer Learning Through Service (LTS), which seems to attract a wider range of students to engineering. LTS programs and courses offer students the opportunity to combine typical learning opportunities with some form of helping and working with others, sometimes for credit. A growing body of evidence advocates that LTS may provide significant advantages to engineering students, giving them a broader perspective and a wider body of knowledge to draw from for future engineering problems, but studies to date are quite limited (Silliman et al. 2010; Bielefeldt et al. 2010; Paterson and Fuchs 2008; Parkinson 2007; Jesiek et al. 2011). Some of the resulting evidence from student LTS experiences provides certain evidence to begin evaluating student motivations. A further look into engineering design motivation could help to address a fundamental question, which remains unexplored: What motivates engineering students to be engaged in engineering design tasks?

This study begins to examine student motivations within three different service-learning programs 1) an on-going longitudinal study on engineering students, 2) an international senior capstone design program, and 3) an international graduate-level research program. As a part of the overall assessment of these programs, the motivations of student participation is reviewed for better understanding of what attracts and retains students within engineering design programs.

\subsection{Intrinsic Motivation}

There are a variety of different kinds of motivation, however; simply focusing on intrinsic motivation, there are multiple aspects that could be explored in order to better understand student motivations for the purposes of exploring engineering design. Intrinsic motivation provides a starting point for understanding why people do what they do, which aids in understanding motivation behind engineering students pursuing design tasks out of personal interest. According to Eccles et al. (2002), intrinsic motivation requires an individual to "do activities for their own sake and out of interest in the activity." Intrinsic motivation can also be seen as something related to opportunity. Engineers have commonly been thought of as problem solvers, which can be attributed to the opportunity to use the knowledge they have.

Flow theory builds upon intrinsic motivation and suggests that only "when a person feels that the opportunities for action in a given situation match his or her ability to 
master the challenges" (Eccles and Wigfield 2002), can they be intrinsically motivated and "flow," for an activity, can be achieved (Csikszentmihalyi 1990). Flow theory may be an appropriate perspective for motivation with regard to engineering design. It also helps to provide a premise for matching abilities with interest, creating a basis for degree selection, which aids in understanding why students may choose to pursue engineering over other degree options.

Others, however, have suggested that there are three kinds of intrinsic motivation: to know, to accomplish, and to experience stimulation (Areepattamannil et al 2011; Vallerand 2002), which all contribute to one's desire to perform a given activity. Engineering students experience all three of these kinds of intrinsic motivation throughout design tasks; they strive to know through initial problem solving stages, they find accomplishment through the creation of a design or prototype in an effort to solve the problem, and they experience stimulation through creating a final product or solution, which solves the problem.

Although intrinsic motivators provide one way of looking for engineering design motivation, it may not be the only possible or the most appropriate, since students may not always view design as an opportunity for action, as a means of matching their ability to master the challenge, or as a way to learn, accomplish, or experience stimulation. Intrinsic motivational theory has diverged into several theories and differentiations. As a result the perspective from which to discuss engineering design motivation needs to be further explored.

\subsection{Achievement Motivation}

Similar to intrinsic motivation theorists, achievement goals theorists attempt to incorporate the need for accomplishment with people's choice with respect to tasks and broaden it to understand their motivation (Eccles et al. 1998). Motivational theories attempt to explore choices and venture to explain questions like how, what, and why efforts are achieved (Matusovich et al. 2009). Eccles et al. (1998) have attempted to define achievement motivation as one related to performance. Engineering design is rich with tasks and performance. Students are often required to complete specific tasks with a rewards system in place, often grades, but sometimes client satisfaction, acting as the reward. Such reasoning is the premise on which Matusovich et al. claims engineering education research could utilize the achievement goal theory to motivate engineering students. Achievement goals are the basis for the attitudes instrument within the survey utilized in the on-going longitudinal study in engineering students, further discussed in later chapters (Pieper 2003; Finney et al. 2004; Miller 2008). Some of this work has suggested that mastery and performance are key learning goals that motivate individuals to achieve goals. These goal theories have evolved in two similar directions.

The first has created three goal related orientations: ego-involved, task-involved, and work avoidance (Nicholls et al. 1990). These premises are based off of ideas like those outlined in intrinsic motivation: interest, desire to know, and problem solving. Similar to the intrinsic motivator, the need to accomplish, task-involved orientation encompasses the idea of self-defined success as a result of accomplishing something, whereas ego- 
involved goals requires individuals to ascertain superiority over others with their abilities. Focusing on these ideas with respect to engineering students, they work to master the tasks they are given as part of a personal interest of problem solving through which they want to do well (performance).

Work-avoidance, on the other hand, overlaps with the second direction these goal theories have taken. Nicholls et al. suggest work-avoidance to be related negatively towards tasks, including those related to task and ego-involved orientations. Pieper, however, felt work-avoidance could be worked into the current four-factor structure, but as the fifth factor linked with mastery and performance goals (Pieper 2003). This idea resulted from previous work deeming mastery and performance had secondary dimensions approach and avoidance (Ames 1992). The 2 × 2 matrix idea of masteryapproach, mastery-avoidance, performance-approach, and performance-avoidance was the stepping-stone to adding in this fifth factor (Miller 2007; Elliot 1999; Pintrich 2000; Elliot and McGregor 2001). This five-factor approach was used in the survey student participants completed. These five-factors were utilized as independent variables for the purposes of the logistic regression, which is further described in the results section of the ISES chapter (Chapter 3).

As these factors are related to intrinsic motivation, a logistic regression was performed to demonstrate a correlation with the engineering design motivation (Chapter 3 ).

Although these studies provide five factors with which to evaluate student achievement motivation, little work has used them for understanding students in engineering programs.

\subsection{Gender Influences}

In order to begin to overcome the struggle of attracting and retaining women to engineering disciplines, a better understanding of what is encouraging or discouraging them to stay is a starting point. A study conducted on freshmen and seniors contends that lower levels of confidence in women contribute to their attrition in engineering disciplines and careers (Cech et al. 2011). It additionally suggests that mastery of skills aids in becoming a successful professional, yet confidence is also considerably linked to engineering persistence. This study helps to exemplify methods of analyzing gender differences in engineering disciplines, which aids in determining what motivates females to participate while providing exploration of emergent questions; e.g. are engineering females more motivated than males?

This study makes a case for working to increase confidence for better retention of females, yet it also lacks evidence linking mastery of knowledge and skills to the motivation of pursuing engineering programs. However, a study done on first-year students at seventeen institutions found lower confidence levels influencing female engineering participants, many rooted in their background knowledge and skills (Besterfield-Sacre et al. 2001). This study adds to the gender difference exploration, and provides a basis for beginning to understand knowledge and skills in engineering students. Examining knowledge and skills helps to create supporting questions; e.g. does knowledge and skills impact motivation to do engineering design work? Unfortunately, 
this study on first-year students was unable to determine what the effects of their confidence level in knowledge and skills had on their undergraduate engineering experience. This study also lacked in determining what factors other than skills contributed to motivation for persistence in engineering.

A six-year longitudinal study determined that factors contributing to women staying in science and engineering included positive influence of faculty and career opportunities. Additionally, the main reasons women gave for leaving the science or engineering field was losing interest, being attracted to another major, or being discouraged by low grades (Brainard and Carlin 1997). Brainard and Carlin also addressed the concept of the influence of confidence levels amongst their study's participants, which they found as being related to levels of interest in coursework and positive relationships with faculty, as well as others. Some of these findings imply a certain level of confidence, as well as other contributing factors, are needed to feel successful in the engineering field. This six-year longitudinal study helped to provide a long-term perspective on gender differences and on what is contributing to attrition, which helps to begin to offer some insight on what is demotivating female engineering (and science) students. Additional questions result from examining this study pertaining to what is motivating those who are staying in engineering (and science for this study's purposes). Although this study provided a multitude of factors contributing to student persistence, it additionally did not address the concept of student success as a result of these factors leading to student persistence or motivation associated with persistence.

In a five semester longitudinal study of engineering student performance and retention from five chemical engineering courses, Felder et al. (1995) found student academic success was associated with external help and personal interactions (for females), as well as teaching others (for males). This study additionally examined levels of anxiety and confidence. The results showed that women in the study were entering with less confidence and a higher level of anxiety in comparison to men. As the study progressed, participant criteria (for both males and females) for satisfaction had experienced some decreases, suggesting that students had begun to appreciate an increase in difficulty or a decrease in confidence. This study helped begin to explore areas of success and anxiety with respect to gender differences in engineering students, which additionally created an opportunity to examine aspects that contributed to motivation. However, this study lacks some implications of how these attributes are connected to engineering coursework and motivations.

A study done on 1,387 first-year engineering students found "drive or motivation" toward success to be a common factor affecting confidence in success, with respect to a specific course (Hutchison et al. 2006). This study focused on obtaining information about gender trends impacting confidence in success, which helps in linking engineering coursework to motivations for engineering. Additionally, specific factors pertaining to confidence in success were explored, which resulted in a better understanding of student satisfaction. This study helps to provide additional perspective on aspects that support positive emotions associated with engineering, which trends toward motivation. Further 
examinations of similar common factors affecting student motivations to do engineering design work seem necessary to expand on this study. Although this research provides a basis for linking motivation and confidence in success to engineering coursework, it additionally overlooks how level of education impacts these factors, as well as how they impact students' motivation to pursue engineering design tasks.

\subsection{Educational Influences}

\subsubsection{Educational Influences}

Stake and Hoffman (2001) evaluated the role women's studies played on students' social attitudes, activism, and personal confidence. The study examined gender and educational level, and included teachers and graduate students in addition to undergraduates, who were predominantly women. Some of the results imply that the women's studies classes influence student attitudes and social activism, which are general goals of higher education. A better understanding of what is motivating students in higher levels of education resulted from this exploration in women's studies.

The APPLES study gives an additional viewpoint on engineering student experiences, which provides some insights on motivation of engineering students, at both freshmen and senior levels (Sheppard et al. 2010). The study suggests some similarities between both levels of education and that the motivations may actually be a result of experiences prior to college. Although this study gives some perception on motivation to study engineering from different levels of education, it does not discuss motivation to do engineering design work. Both of the Stake and Hoffman, as well as the APPLES studies, provide some insight on higher education from a women's studies', as well as a larger engineering survey's perspective, yet leaves openings for other studies to further evaluate other varieties of education in how they impact and motivate engineering students.

\subsubsection{Topical Knowledge: Sustainability}

Sustainable practices are becoming an increasingly popular topic in education. These practices are being taught to ensure environmental, economic, health, and social impacts are considered in every step of problem solving, even in the field of engineering. An evaluative study was constructed to determine if sustainable engineering education via service learning results in positive outcomes in engineering students (McCormick et al. 2010). This study worked to evaluate students involved, as well as those not involved, in service learning on three bases: sustainable engineering design, self-efficacy, and epistemological beliefs. The results of this evaluative study helped to create a two-part methodology and a plan to be implemented. The use of these methods was further examined in an assessment done on students involved in service learning (McCormick et al. 2011). The online survey, along with an open-ended reality question developed, provided a means of better evaluating students' understanding of sustainable engineering. This study offers an initial basis to understand the effects of sustainable engineering aspects on students and creates an opportunity to explore this further with students who are participating in sustainable engineering programs. Additionally, an evaluation of what is affected by changes in student perspectives associated with 
sustainable engineering creates an opportunity to determine if it is a contributing factor for students' motivations to do engineering design work. Although this instrument is still in the development stages, some conclusions were reached suggesting higher education should work to incorporate sustainability topics. Similar to this evaluation, the need for sustainable thinking was examined in undergraduate engineering curricula (Huntzinger et al. 2007). This evaluation explored various options of integrating sustainable engineering education, which resulted in suggestions for redesigning of programs for better infiltration of sustainable engineering knowledge. These studies provide a basis for understanding the importance of sustainable engineering and how it can be evaluated within service learning programs as well as within the curricula. Additionally, the aforementioned studies help to imply students are better able to do engineering work, yet leave opportunity for other evaluation on the motivation of participants in these kinds of service learning and engineering design programs.

\subsubsection{Character Development: Cultural Awareness}

Additional studies have been done to determine what activities and programs in college help to develop students' character, yet still prepare them to work in an increasingly diverse world. One study done evaluating college and character determined opportunities that enabled diverse interactions through experience (i.e. within assignments, service learning, or other involvements) with issues relevant to today's society are important for character development (Kuh and Umbach 2004). This evaluation of character development piggybacks on what motivates students by suggesting opportunities and diverse interactions enable students prepare them to work in their respective field. Feeling prepared to do work of interest supports positive feelings associated with performance, as outlined by achievement goal theory. Opportunities for understanding how these activities and programs in college are motivating students result from this study and suggest methods of retaining and attracting students to engineering programs. An additional study evaluating impacts of college on character development found exposure to interdisciplinary and ethnically diverse, as well as gender-diverse groups, are critical to development (Astin and Antonio 2004). Both of these character development studies help to suggest the need for intercultural understanding, possibly it's through cultures pertaining to gender or ethnicity, in order to have a good understanding of today's society exposure to the kinds of groups are essential. Additionally, these studies suggest that an intercultural understanding helps prepare students, which also provides a means of motivating students. Linking this idea to engineering programs could help to determine what is motivating students to pursue engineering design tasks. An additional study was conducted on how to measure intercultural sensitivity (Bennett 1993; Hammer et al. 2003). The measurement helps to address how students are developing their intercultural understanding. The results of this instrument provide a means of suggesting where student understanding lies on a spectrum, ranging from denial/defense to acceptance/adaptation. The study implies, however does not directly support, that higher scores closer to acceptance/adaptation are more desirable to suggest a better cultural understanding. Linking this study to the previous studies helps to suggest that a better understanding of cultural awareness prepares students to work in their field, which could increase motivation as a result. This promotes the question; does greater intercultural 
awareness motivate students? These studies help to support programs involving intercultural, as well as international experiences, yet do not directly discuss how student involvement in specific degree programs, like engineering, benefit from their motivation to continue pursuing their respective program (i.e. engineering).

A study done on undergraduate and graduate engineering students participating in a 10week international experience in China helps to begin address the benefits concerning involvement in international experiences (Jesiek et al. 2011). Participants within this study were required to complete a series of surveys and evaluative instruments at various phases of their experience. These helped to examine how prepared students were to participate, as well as what they were gaining through their involvement, which also provided support for international experiences in engineering programs. Evaluating students' self-assessed level of preparedness to participate in a given program provides insight on how well students feel they will be able to perform in their respective program. This understanding also links to interest and motivation, as outlined by flow and achievement goal theories. This information can also be used in better understanding what motivates students in engineering programs, where applied. It suggests the question of whether student self-reported level of readiness is an indicator of their motivation. An additional study on undergraduate engineering students began to address participant readiness to do self-directed learning, which offered additional evaluation for students pursuing alternative work in their engineering education (Litzinger et al. 2005). The results of this examination suggest that these kinds of experiences must be carefully crafted to ensure students at the various levels of readiness have the necessary support to succeed. This study additionally links readiness and cultural understanding, suggesting that they are both contributing factors to their level of success. As previously discussed, success is thought to be linked to student motivation, yet findings still need resolution. Although, these studies provide a basis for supporting intercultural competency with increased focuses on preparedness and some evaluation of international program involvement, they leave opportunities for evaluating these concepts, among others, with respect to motivation for participation in these kinds of engineering programs.

\subsection{Engineering Design Motivation}

Because the literature suggests there are various aspects and definition for motivation, specific focus and evaluation will be given to Carberry's self-efficacy instrument, which evaluates motivation, among three other concepts; success, anxiety, and confidence (Carberry et al. 2009; 2010). Questions pertaining specifically to engineering design tasks were asked within the instrument and students were asked to respond based on how motivated they would be in performing the task on a 0 to 100 Likert-type scale. This instrument not only focuses on motivation to perform specific tasks, it incorporates engineering design, which is a limited trait within the previously outlined studies within the literature. The instrument is currently used in the Impacts of Service on Engineering Students survey (discussed in later chapters), which is implemented for all three of the aforementioned studies in this thesis. The availability of these results adds to the attractiveness of the instrument, however, the interest in what is attracting and retaining students within the engineering field is what ultimately drives this study. For the 
purposes of the remaining analyses, motivation to perform engineering design tasks will be explored using an average motivation score from Carberry's self-efficacy instrument.

\subsection{Purpose of Study}

The purpose of this study aims to explore what motivates engineering students to complete engineering design tasks. This study works to incorporate both quantitative and qualitative approaches in understanding what can be done to continue to encourage and increase engineering student motivation to do engineering design work. Although the literature provides a basis to better understand differing kinds of motivation, this thesis was written with the intention of evaluating motivation to do engineering design specifically.

\subsection{Research Questions}

Research questions are broken up in a similar way as the assessment methods described in Chapter 2, by qualitative and quantitative categories. The quantitative hypotheses are further examined using statistical analysis and result in some formal conclusion suggesting a claim or recommendation, whereas the qualitative hypothesis are analyzed using information collected in a more ethnographic manner (see Chapter 2 for details) and cannot be statistically confirmed. The details of these questions are further explained below.

\subsubsection{Quantitative Hypotheses}

Quantitative methodologies utilized in their respective program help to examine what motivates students to do engineering design tasks by evaluating the following hypotheses:

1) Female students are more motivated to participate in engineering design programs when given the opportunity to apply what they are learning to help others (Felder et al. 1995; Brainard and Carlin 1997),

2a) A greater level of knowledge and skills provides a basis for which students increase their motivation as a result of participating in engineering design programs (Besterfield-Sacre et al. 2001; Vallerand 2002; Areepattamannil et al 2011; Cech et al. 2011),

2b) Students with higher levels of mastery and performance approach attitudes will have higher levels motivation to do engineering design work than those with higher levels of avoidance attitudes (Ames 1992; Eccles et al. 1998; Elliot 1999; Pintrich 2000; Elliot and McGregor 2001; Pieper 2003; Finney et al. 2004; Miller 2008; Miller 2007),

2c) A greater sense of personal satisfaction associated with students' self-reported level of success and incentive to complete engineering design tasks leads to higher motivation (Felder et al. 1995; Hutchison et al. 2006),

2d) Students with higher self-reported levels of success are more motivated to do engineering design tasks (Felder et al. 1995; Hutchison et al. 2006), and

2e) A greater level of anxiety associated with performing engineering design tasks leads to lower motivation (Felder et al. 1995). 
The remainder of this thesis will work to address these hypotheses using subsequent assessment methods outlined in Chapter 2.

\subsubsection{Qualitative Hypotheses}

Additionally, qualitative analyses provide some insights on perspectives, which cannot be analyzed statistically. Valuable data was collected within the aforementioned programs, yet some is in too early of stages to be statistically analyzed at this point. Analyses of this information aims to address the following hypotheses:

3) Students who self-report higher levels of readiness to participate in their respective engineering program are more motivated with respect to engineering design tasks (Litzinger et al. 2005; Jesiek et al. 2011),

4) Students with higher levels of intercultural competency are expected to have a better understanding of other cultures, leading them to be better equipped and more motivated to perform engineering design tasks in other societies (Hammer et al. 2003; Astin and Antonio 2004; Kuh and Umbach 2004),

5) A better understanding of sustainable engineering should result in higher levels of student motivation to utilize these skills with regard to engineering design (Huntzinger et al. 2007; McCormick et al. 2010; McCormick et al. 2011),

6) Highly educated students are motivated to do engineering design tasks at higher levels when utilizing their skills (Stake and Hoffmann 2001; Sheppard et al. 2010), and

7) Individuals who have previous international experiences are more motivated to pursue others where they can additionally utilize their engineering design skills (Hammer et al. 2003; Kuh and Umbach 2004; Jesiek et al. 2011).

Additionally, some observational information pertaining to the collected data incorporate an opportunity to address the aforementioned hypothesis:

1) Female students are more motivated to participate in engineering design programs when given the opportunity to apply what they are learning to help others (Felder et al. 1995; Brainard and Carlin 1997).

Methods utilized in this process are outlined in Chapter 2 and further detailed in subsequent chapters where applicable. 


\section{Assessment Methods}

Due to the nature of the information being collected for this study, both qualitative and quantitative methods have been utilized to evaluate all of the data. The instruments and methodology are outlined below and detailed within other chapters where they are utilized. Although various instruments are available to assess programs similar to those discussed in this thesis, the methods described below were selected specifically to address the aforementioned hypotheses and objectives (Chapter 1).

The instruments outlined in Section 2.1 will be utilized to determine what is motivating students to do engineering design work. Four instruments -Readiness (Section 2.1.1), Intercultural Development Inventory (Section 2.1.2), Impact of Service on Engineering Students (ISES, Section 2.1.3), and Sustainable Engineering via Service Learning (SESL, Section 2.1.4) - use Likert-type scales to tabulate student self-reported scores, therefore each will be considered a component of the quantitative evaluation of students. Additional priority is given to the ISES survey, as it is comprised of multiple validated instruments. ISES also provides scores for student motivations to do engineering design, which are utilized throughout the remainder of this thesis. Results of these instruments are intended to support one another to demonstrate an increase in understanding in areas that may be useful in motivating students to do engineering design work. These instruments are used to evaluate the programs within this thesis.

Qualitative information can often provide a much richer perspective on the details of the data as compared to their quantitative counterparts. Two qualitative instruments motivational narratives and interviews - are used in this work. These instruments are additionally meant to support the quantitative data and provide detailed information to answer questions that result for the numerical information.

Table 2.1

List of Assessment instruments

\begin{tabular}{|l|}
\hline \multicolumn{1}{|c|}{ Instruments } \\
\hline Readiness Assessment \\
\hline Intercultural Development Inventory \\
\hline ISES (Motivation) \\
\hline SESL \\
\hline Word Cloud \\
\hline Motivational Narratives \\
\hline Interview Protocol \\
\hline
\end{tabular}




\subsection{Quantitative Methodology: Assessment Instruments}

Although rich data results from the interviews, there is still a need for quantitative analysis. Instruments utilized within each section are initially outlined using a table similar to Table 2.1. This section will explain the prioritization, utilization of instruments, data processing, as well as means of utilizing similar methods in future work.

Various instruments, described below, are incorporated in this study in order to address specific targeted outcomes of the individuals participating in community engagement programs. The following methods provide insight into student motivations pertaining to engineering design work (Table 2.1). However, the wide range of information available makes other explorations possible for future endeavers.

\subsubsection{Readiness to Serve: Readiness Inventory}

The readiness assessment is based off of the Miville-Guzman University-Diversity Scale (MGUDS) (Milville et al. 1999). It is comprised of twenty questions from the original forty-five item instrument and utilizes a six-point Likert-type scale, which ranges from strongly agree to strongly disagree. It has been used to examine engineering students' awareness and potential acceptance of cultural similarities and differences (Jesiek et al. 2011). This instrument provides one lens on the motivations of students participating in the community engagement programs being assessed. The resulting information provides essential background information and further perspective to analyze the students' motivations.

Students are asked to complete the readiness assessment as they begin the program and prior to departing for fieldwork. Their readiness scores provide background information to begin determining whether student feel prepared to participate in fieldwork. This assessment adds depth to their motivational responses (i.e. motivational narratives and motivation scores) and helps to paint the picture of how students are prepared and perhaps how motivated they are to participate.

In order to score individual student responses, a method was developed using a six-point Likert-type scale ranging from Strongly Disagree to Strongly Agree, -3 to 3 (zero was omitted). For proper summation, negatively worded questions (numbers 1, 5, 10, 12, 13 and 15 were inverted) were inverted. Student self-reported scores were tabulated and summed to result in an overall readiness score. A threshold of zero was set to determine if students taking this assessment self-report themselves as prepared to participate in their respective program. This is further discussed in Chapters 4 and 5. A sample of questions in the Readiness Inventory can be found in Appendix 7 Figure 7.1.

\subsubsection{Intercultural Awareness: Intercultural Development Inventory}

The Intercultural Development Inventory (IDI) is a commercially available, validated, and extensively used instrument to assess intercultural competency (Bennett 1993; Hammer et al. 2003). It is comprised of fifty Likert-type questions disseminated through an online survey format. Students are asked to take this instrument prior to starting the program, before fieldwork, and upon return to campus. This tool provides insight on 
how well they might work with others of different backgrounds, including aspects like culture and worldview, which may be considered an indicator for student motivations with regard to these kinds of programs. The results of this assessment give two scores: a perceived and a developmental (actual) orientation. Such scores are used to place the individual on a scale ranging from ethnocentric, a more mono-cultural orientation, to ethnorelativistic, a more adaptable state (Figure 2.1). These scores can provide additional information for understanding student motivations while participating in these programs.

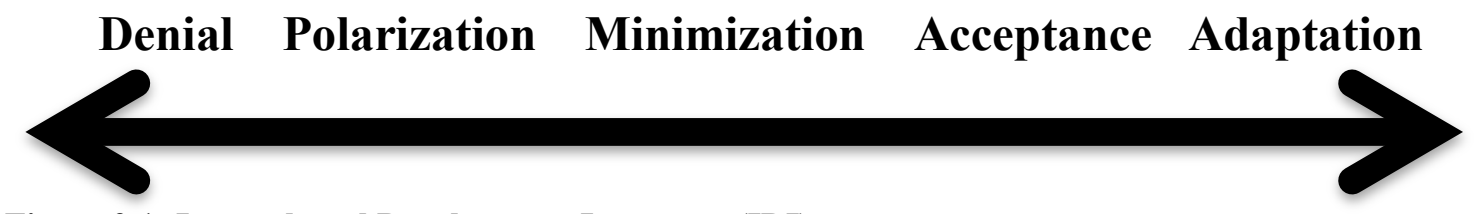

Figure 2.1: Intercultural Development Inventory (IDI) spectrum

\subsubsection{Knowledge, Skills, and Attitudes: Impacts of Service on Engineering Students Survey}

The Impacts of Service on Engineering Students (ISES), a three-year on-going longitudinal study further detailed in Chapter 3, utilizes an online survey comprised of four main instruments consisting of 131 questions. Student skills and attitudes are evaluated using multiple validated instruments within the ISES survey, they include; 1) Engineering design self-efficacy (Carberry et al. 2009; Carberry et al. 2010), 2) Attitudes towards learning (Finney et al. 2004), 3) National Engineering Students' Learning Outcomes (Pierrakos et al. 2008a; Pierrakos et al. 2008b; Pierrakos et al. 2007), and 4) Measures of well-being (Keyes 2006). Many of these instruments utilize a Likert-scale or Likert-type scale to evaluate students. They are all implemented in the form of one online survey, which result in student self-reported scores. Students are asked to complete this assessment upon entering the program and at additional subsequent times depending on which program they are involved with. The survey takes about thirty minutes. The questions prompt responses to program involvement and require students to provide feedback on improvements on their knowledge, skills, professional and personal experiences all based on participation in the program. This survey also provides insight on student motivation in a variety of ways within each instrument. For the purposes of this analysis select questions pertaining to motivation were utilized in data analyses. Select sample questions can be found in Appendix 7 Figure 7.2.

Chapter 1 detailed the use of the Carberry et al. instrument $(2009 ; 2010)$ and how it is specifically utilized to evaluate motivation to do engineering design work. Carberry's instrument focuses on confidence, anxiety, success, and motivation related to engineering design self-efficacy (Carberry et al. 2009; Carberry et al. 2010). Specific to motivation, the available nine questions were averaged to create one engineering design motivation score. This score is utilized as a measurement within all three of the program studies presented in Chapters 3, 4, and 5. For the purposes of analyzing pre and post scores the cohort's average is utilized as a threshold to determine high from low levels of motivation. This is done primarily due to the nature of the Likert-type scale 
associated with student responses, 0 to 100. Participants completing this survey are all engineering students who are used to being evaluated on a 0 to 100 scale for the purposes of exams, quizzes, and homework. Their perspective is slightly skewed in that the median, 50, is often considered failing. In order to address this skewed perspective, the average is utilized as the threshold score differentiating high from low. Motivation score was used as the dependent variable in a logistic regression (Chapter 3 ) to help answer some of the aforementioned hypothesis pertaining to engineering design motivations. Further details are provided in subsequent chapters.

\subsubsection{Sustainable Engineering Knowledge: SESL}

The Sustainable Engineering through Service Learning (SESL) instrument is a validated survey by McCormick et al. (2010). SESL works to address the awareness and attitudes of students to work on sustainability issues; as such it could provide topical insight on motivation of students. It is commonly thought that the better understanding one has of a given area, the more prepared and motivated they are to participate. This is an online survey comprised of thirty-five Likert-scale questions, which examine student selfefficacy, beliefs and knowledge on sustainable engineering topics (McCormick et al. 2010; McCormick et al. 2011). A second component of the SESL instrument is a written response to an open-ended challenge question on an engineering problem. For this component students can choose from two different scenarios and respond with how they would go about solving the problem. Their responses to these two components provide detailed information about their sustainable engineering mindset. From this assessment a better understanding of the students, possible explanations of their sustainable engineering mindset in relation to international service, as well as the effectiveness of the programs in which they were involved can be examined. This also gives insight on how the program does in preparing the students for their fieldwork. Students are asked to complete these instruments upon entering the program and prior to departing for fieldwork, as well upon return. A sample of these questions can be found in Appendix 7 Figure 7.3

\subsubsection{Motivations Narratives: Frequency Analysis}

The motivational narratives are utilized to better understand why students are participating in their respective program. Students are given a prompt (Appendix 7 Section 7.1) and asked to write approximately a page about why there were motivated to participate in their respective program, further described in section 2.2.3. This data can provide some essential information for qualitative analysis; however, it can also yield quantitative information. After coding, narrative statements were analyzed using a frequency report tool built into HyperRESEARCH ${ }^{\circledR}$ to examine thematic tendencies in student responses (ResearchWare, Inc. 2010). Chapters 4 and 5 discuss how the narratives were coded in greater detail. The dataset can be examined using several filter options (all responses, by gender, class ranking, intercultural experience, etc.). This information provided a basis with which to start the quantitative analysis described in later chapters.

\subsubsection{Interview Protocol: Frequency Analysis}

Similar to the motivational narratives, interviews can provide information for both 
qualitative as well as quantitative analysis. Students were asked questions related to the survey questions, but more in-depth. Additionally, these interviews can provide some insight on fieldwork components of programs discussed in Chapters 4 and 5, which can provide a better framework for which to determine further understanding of student motivations to do engineering design work. Sample interview questions can be found in Appendix 7 Section 7.2. Interviews utilized in studies discussed in later chapters also used HyperRESEARCH ${ }^{\circledR}$ to code and analyze the flagged text (LeCompte and Schensul 1999; Gibbs 2007). This provided a means to convert some of the qualitative information to a quantitative format. This data supplements the findings gathered through the survey questions and supports the conclusions resulting from the data analyses.

\subsection{Qualitative Methodology}

The richness in interview data is very difficult to obtain using quantitative methods alone. In order to gather the more in-depth information, options outside of the quantitative perspective needed to be explored. The methodologies utilized are further detailed below.

\subsubsection{Ethnographic Methodology}

The primary method for dealing with interview data is ethnography. According to Lindlof and Taylor (2011) ethnography is "a genre of qualitative research" which is linked to recording and working with communicative information. For the purposes of the collected interview data a more ethnographic approach is being taken by utilizing semi-structured interviews along with the coding and analysis methods. The semistructured and somewhat open-ended questions within the interviews enabled the participant to share information in a less demanding manner leading to furthered learning about the individual and the program they are involved with. Although there are a variety of ways in which the transcribing and coding processes can be done, the focus of the efforts for this study were on the actual spoken words and less on the tone behind what was being said. As interviews were over the phone, body language was not analyzed. Every word was transcribed to the best of the transcriber's ability (Kvale and Brinkmann 2009). Certain leeway was given for mumblings and cut-outs in the audio file; in these instances the transcriber would denote this in some form. Additionally, the process by which codes were assigned was based off of a thematic in vivo methodology (Saldaña 2009). Within subsequent chapters the process in which these methods are utilized are further discussed.

\subsubsection{Word Cloud}

In order to best exemplify common codes and phrases found within the motivational narratives, as well as interview responses Word [tag] clouds were utilized. Word clouds, as demonstrated in Chapters 4 and 5, help to visualize the frequency with which words were stated in a given set of text. Sinclair's study examined how users preferred to search for information between word clouds and search boxes (2008). It suggested that these word clouds could be utilized over search boxes to help users find specific information, but also for browsing purposes. Additionally, some results determined the clouds could be used as a visual summary, which could be utilized by anyone, including 
those who speak English as a second language. This perspective was utilized in initially evaluating the motivational narratives, further explained below. It provided a better understanding of what common motivational factors were apparent for the participants. Chapters 4 and 5 further outline the results and use of this method.

\subsubsection{Motivational Narratives}

Additionally, for some of the programs discussed in later chapters, students are asked to write a description of their motivation for participating in the program. These narratives are examined to determine the top motivations for participating in the program. This information can be rich in detail and provide insight for individuals that would be difficult to gather in other methods. Prior to starting the program, students were given the following prompt:

Task: Write a narrative, no more than one page at 12 point font, describing your motivations for wanting to participate in this program. Print out, staple to this cover sheet, and drop off (Appendix 7 Section 7.1).

As standard protocol, no names are allowed on returned responses; rather student's use a six-digit codename (first 2 letters of first name + first 2 letters of last name +2 numbers from birthday) across all five instruments (Guzak and Paterson 2011: Guzak et al. 2012).

Each motivations narrative essay was transcribed, then coded using qualitative data analysis software (HyperRESEARCH ${ }^{\circledR} 3.0$ ), bearing in mind the question: Why are students interested in participating in these programs? Appendix 8 Table 8.1 includes the list of codes created, including further explanations of each.

\subsubsection{Interview Protocol}

In addition to the survey and narrative instruments, some students were asked to participate in the interview protocol, as mentioned previously (Appendix 7 Section 7.2). This process can either be in the form of a phone or in-person interview lasting approximately thirty minutes in length. Students are asked similar, follow-up questions to the survey instruments for information providing further insight on student selfreporting, some of which pertain to their motivations. At this point, this method is in the beta testing phase with the hope of implementation for all Michigan Tech community engagement programs in the future, however, it is utilized extensively in the longitudinal study, further discussed in Chapter 3.

\subsection{Program Overview}

As Chapter 1 began to outline, LTS are becoming more common in engineering programs across the nation. Learning Through Service provides an opportunity to apply traditional learning to service projects. This study presents some initial findings of student motivations within three different Learning Through Service programs: 1) an ongoing longitudinal study examining the Impacts of Service on Engineering Students (ISES), 2) an international senior capstone design program (idesign), and 3) a graduatelevel Peace Corps Master's International program (PCMI). The first study incorporates 
individuals across the spectrum of service involvement (none to frequently), although the focus will be on those individuals' motivations and not their involvement in service based projects. The second program uses the traditional capstone design template in an international community where students design for the community to which they are assigned. Lastly, the third program creates the opportunity to serve in the Peace Corps for the research component of a M.S. degree. These programs are further described in later chapters.

Although this study focuses on engineering design motivation for students involved in the aforementioned programs, none of the assessment protocols for these programs were designed with the sole purpose of examining motivations. The nature of all three of these programs was designed to gather a broader array of information to provide a framework of understanding of engineering and community engagement. Nevertheless, they provide an excellent opportunity to evaluate the engineering design motivation of the participating students. Subsequent chapters will present the utilization of these assessment methods and findings of these on-going efforts. 


\section{Impacts of Service on Engineering Students}

\subsection{Background}

The Impacts of Service on Engineering Students (ISES) study is a collaborative effort between primarily four universities (Michigan Tech University, Tufts University, James Madison University, and the University of Colorado Boulder) to craft an additional subgroup composed of Engineers Without Borders (EWB) students from across the country (twenty institutions). This project is funded by the National Science Foundation (NSF EEC-1025220). This study aims to examine the effects of service on engineering education by looking at engineering students over a three-year longitudinal study. Students enrolled as freshmen or juniors were selected in an effort to get the whole picture of engineering education, from freshmen year all the way to placement in the workforce or post-secondary education over a shorter period of time. Quantitative online survey questions and qualitative interview questions were incorporated to evaluate student experiences with their respective engineering curriculum. An interest in obtaining a better understanding of why students want to do engineering with the longer term goal of attracting more students lead to the development of the aforementioned ISES survey. Within this instrument, using questionnaires and interviews, students are asked questions rooted in motivational analysis (among other topics), which provided the basis for the narrowed focus within this portion of this thesis.

\subsection{Program Description}

Overall, the ISES study aims to better understand the impacts of service on engineering education. However, to address the reoccurring question of what motivates students as a means of retaining them in the engineering field, this chapter begins examining student motivations with respect to engineering design. The results section discusses the findings of the multinomial logistic regressions run on the data from all of the study's participating students.

The goals outlined for the overall ISES study differ from the goals of the study done for this thesis; they include the following hypotheses:

1)Learning Through Service (LTS) increases the professional skills required for holistically-thinking engineers without decreasing technical capability,

2) Attributes of holistically-thinking engineers are measureable via combined assessments of technical skills and self-efficacy, identity, attitudes, and other psychosocial factors,

3) Extracurricular LTS efforts, such as EWB, and curricular LTS efforts provide the same benefit; i.e., there is no discernible difference in impacts from different forms of LTS, and

4) Underrepresented students are attracted to, retained in, and persist through engineering programs at higher levels when engaged in LTS (Parkinson 2007; Paterson and Fuchs 2008; Silliman et al. 2010; Bielefeldt et al. 2010; Jesiek et al. 2010). 
All of these goals will eventually be addressed upon completion of the 3-year period, however, for the purposes of this thesis, only portions of some of these hypotheses were utilized. These newly created hypotheses are further explained below.

The previously outlined hypotheses from the ISES proposal were utilized to craft the following subset of qualitative and quantitative hypotheses. These were previously mentioned in Chapter 1(numbered in the same manner), and will be addressed throughout the remainder of the chapter to better understand student engineering design motivations. These include:

1) Female students are more motivated to participate in engineering design programs when given the opportunity to apply what they are learning to help others (Felder et al. 1995; Brainard and Carlin 1997),

2a) A greater level of knowledge and skills provides a basis for which students increase their motivation as a result of participating in engineering design programs (BesterfieldSacre et al. 2001; Vallerand 2002; Areepattamannil et al 2011; Cech et al. 2011), 2b) Students with higher levels of mastery and performance approach attitudes will have higher levels of motivation to do engineering design work than those with higher levels of avoidance attitudes (Ames 1992; Eccles et al. 1998; Elliot 1999; Pintrich 2000; Elliot and McGregor 2001; Pieper 2003; Finney et al. 2004; Miller 2008; Miller 2007), 2c) A greater sense of personal satisfaction associated with students' self-reported level of success and incentive to complete engineering design tasks leads to higher motivation (Felder et al. 1995; Hutchison et al. 2006),

2d) Students with higher self-reported levels of success are more motivated to do engineering design tasks (Felder et al. 1995; Hutchison et al. 2006),

2e) A greater level of anxiety associated with performing engineering design tasks leads to lower motivation(Felder et al. 1995), and

6) Highly educated students are motivated to do engineering design tasks at higher levels when utilizing their skills (Stake and Hoffmann 2001; Sheppard et al. 2010). The analyses of these are further explained in this chapter.

\subsection{Program Assessment}

The quantitative and qualitative methods outlined in Chapter 2, also outlined in Table 3.1, were and continue to be utilized in the assessment of the Impacts of Service on Engineering Students. These methods are intended to help evaluate engineering design motivations for participants in the ISES study. The remainder of the chapter discusses the utilization and results of these methods. 
Table 3.1

Assessment instruments use in the Impacts of Service on Engineering Students research project

\begin{tabular}{|l|c|}
\hline \multicolumn{1}{|c|}{ Instruments } & ISES \\
\hline Readiness Assessment & \\
\hline Intercultural Development Inventory & $\mathrm{X}$ \\
\hline ISES (Motivation) & $\mathrm{X}$ \\
\hline SESL & \\
\hline Word Cloud & \\
\hline Motivational Narratives & $\mathrm{X}$ \\
\hline Interview Protocol & \\
\hline
\end{tabular}

\subsubsection{Quantitative-Online Surveys}

Engineering students from the aforementioned universities and the EWB organization were initially asked to complete an online survey expressing interest in participating in the study. From the gathered responses, a pool of 254 students were selected, eliminating students who were not freshmen or juniors at the time. Due to this study's longitudinal nature, these students have since been asked to complete online surveys comprised of questions from previously validated instruments to evaluate student selfreported scores, including responses to motivational questions.

Initially, in Spring 2011, the surveys were comprised of 131 Likert-scale and Likert-type scale questions, with an additional nine questions were for demographics, four of which were open-ended. These questions were selected utilizing the outlined validated instruments previously mentioned in Chapter 2 (section 2.1.3). The demographic questions are used as identifiers as well as additional independent variables; for example, the gender question provides a basis for hypotheses $2 \mathrm{a}$ and $2 \mathrm{~b}$, outlined in Chapter 1. In Fall 2011 the survey was condensed to fifty-seven from the original 131 overall questions with twenty-two work-career-life and six demographic additional Likert-type questions, as well as three open-ended questions. This reduction was based off of random selection in an effort to reduce the attrition rate. However, for Spring 2012, factor analysis was utilized to determine if the random selection overlook valuable questions. This process uses factor analysis within the principal component analysis option in SPSS ${ }^{\circledR}$ to reduce the number of variables based on those having an Eigenvalue greater than one, which separates the questions into factors (Appendix 9). Further alterations were made to the survey, adding back in some questions that were dropped in the fall survey. This resulted in 101 overall questions, eight Likert-type questions, and four open-ended demographic questions for Spring 2012. These questions will be used for the remainder of the ISES study (Table 3.2). Appendix 10 Section 10.1 details the processing of this data for further analysis, which is outlined in the results section.

Additionally, the aforementioned engineering design motivation score, developed by Carberry (Carberry et al. 2009; Carberry et al. 2010) and detailed in Chapter 2, is further utilized for the purposes of analyzing ISES participants. The results section depicts the results of ISES participant scores utilized in the logistic regression. 


\subsubsection{Qualitative-Interviews}

As mentioned previously, a subset of students $(n=121)$ were asked to participate in phone interviews lasting approximately thirty minutes. Students were selected so half the pool would be juniors and the other half would be freshmen. Additionally, the initial intent was to have population with one-third of students having no LTS experience, onethird only having curricular service learning (CSL) experience, and one-third only having extracurricular service learning (ESL) experience. Unfortunately, due to the limitations of the volunteer participants and engineering curricula at target institutions, one-third of students with only curricular service experience was not an option; a majority of community engagement is extracurricular. This pool was modified so that as many of these students as possible had CSL, however, several ended up with both CSL and ESL, and a few had only ESL experiences. Demographics of these participants are found in Table 3.3. These same students will continue to be asked to participate throughout the remainder of the study. It is anticipated that students will change their ESL and CSL level of involvement over time and perhaps change categories all together. Of the 121 interviewees forty were selected for this thesis in order to focus on motivations. Understanding this subset of the students within the study should lead to better understanding of the entire group, producing insights on engineering students in general and perhaps leading to better retention of students. The results section further describes some of these findings and trends from the first three semesters of this longitudinal study.

These interviews were somewhat structured in nature, allowing students to have some direction with their responses, but also leeway to expand their thoughts and opinions. Questions focused on areas of engineering student background, attitudes, knowledge, satisfaction, experience, work and life, and mindset; a sample of these questions can be found in Appendix 7.2. The Spring 2011 interviews were the longest, perhaps due to the uncertainty in how long students would take to respond to the questions or due to additional built-in follow up questions. These interviews ended up ranging from 18 to 45 minutes in length. The approximate thirty short-answer and open-ended questions was reduced to approximately twenty-five questions of the same spirit for Fall 2011 interviews, which reduced the duration of interviews to 14 to 39 minutes. This same model was used for Spring 2012, with a few additional questions for clarification that were unavailable in the previous version, resulting in twenty-seven questions (plus the built-in follow up questions), which left the range at 14 to 40 minutes. All 121, 109, and 98 interviews for Spring 2011, Fall 2011, and Spring 2012, respectively, were conducted by the same individual ensuring some form of standardization for this process (Table 3.2). Appendix 10 Section 10.2 outlines the processing of this data for further analysis discussed in the results section of this chapter.

The interviews were transcribed using a combination of Dragon Dictate ${ }^{\circledR}$ and HyperTranscribe $^{\circledR}$ and then coded and analyzed using HyperResearch ${ }^{\circledR}$. Although many methods could have been selected, for the purposes of coding the interviews, in vivo thematic coding was utilized to incorporate as much information in the students' words as possible. The incorporation of general ideas that came as a result of reading through 
some of the text helps to create the overall codes. ".... a theme is a phrase or sentence that identifies with a unit of data is about and/or what it means," (Saldaña 2009,139) is the idea behind which themes were chosen for the purposes of analyzing student motivations. Using words that reflect exactly what the students mean or discusses what their experience was about leading to their motivation are the methods utilized to analyze the information. For the purposes of this paper, questions involving student motivations were further evaluated to determine if there were any general trends for the overall group as well as within the extreme motivational groups; other aspects of the interviews will be examined in future papers. These trends and findings are discussed within the results section.

Table 3.2

Three-semester question breakdown for the Impacts of Service on Engineering Students study

\begin{tabular}{|l|c|c|c|}
\hline & \multicolumn{1}{|c|}{ Spring 2011 } & \multicolumn{1}{c|}{ Fall 2011 } & Spring 2012 \\
\hline \# of Surveyed Students & 254 & 213 & 198 \\
\hline $\begin{array}{l}\text { \# Freshmen/Sophomores/ } \\
\text { Juniors/ Seniors/ } \\
\text { Doing something else/ } \\
\text { Graduated }\end{array}$ & $\begin{array}{c}150 / 0 / 104 / \\
0 / 0\end{array}$ & $\begin{array}{c}0 / 112 / 12 / \\
80 / 4 / 0\end{array}$ & $\begin{array}{c}0 / 87 / 23 / \\
80 / 7\end{array}$ \\
\hline $\begin{array}{l}\text { Avg. GPA } \pm \text { Standard } \\
\text { deviation }\end{array}$ & $3.34 \pm 0.52$ & $3.34 \pm 0.56$ & $3.36 \pm 0.47$ \\
\hline $\begin{array}{l}\text { Major: (BE/CE/ChE/EE/ } \\
\text { EnE/ME/Other) }\end{array}$ & $\begin{array}{c}19 / 37 / 13 / 15 / \\
25 / 62 / 83\end{array}$ & $\begin{array}{c}17 / 37 / 14 / 10 / \\
23 / 51 / 61\end{array}$ & $\begin{array}{c}15 / 38 / 12 / 11 / \\
56 / 66\end{array}$ \\
\hline \# of Interviewed Students & 121 & 109 & 98 \\
\hline \# of Interview Questions & 30 & 25 & 27 \\
\hline \# of Survey Questions & 131 & 57 & 101 \\
\hline \# of Demographic Questions & 9 & 9 & 12 \\
\hline \# of Additional Questions & - & 22 & - \\
\hline
\end{tabular}


Table 3.3

Interview LTS experience demographics

\begin{tabular}{|c|c|c|c|c|}
\hline & ESL & CSL & Both & None \\
\hline \# of Students & 40 & 5 & 37 & 39 \\
\hline \# Freshmen/Juniors & $20 / 20$ & $2 / 3$ & $\overline{18 / 19}$ & $20 / 19$ \\
\hline$\%$ Female & $53 \%$ & $40 \%$ & $51 \%$ & $33 \%$ \\
\hline $\begin{array}{l}\text { Avg. GPA } \pm \\
\text { Standard deviation }\end{array}$ & $3.54 \pm 0.38$ & $3.05 \pm 0.45$ & $3.37 \pm 0.38$ & $\overline{3.42 \pm 0.44}$ \\
\hline $\begin{array}{l}\text { Major: } \\
\text { (BE/CE/ChE/EE/ } \\
\text { EnE/ME/Other) } \\
\end{array}$ & $2 / 4 / 3 / 4 / 5 / 9 / 13$ & $\begin{array}{c}0 / 0 / 0 / 0 / 2 / \\
2 / 1\end{array}$ & $6 / 9 / 2 / 5 / 8 / 7$ & $1 / 6 / 2 / 4 / 6 / 7 / 13$ \\
\hline $\begin{array}{l}\text { University: } \\
\text { (CU/JMU/MTU/ } \\
\text { Tufts/Others) }\end{array}$ & $\begin{array}{c}2 / 8 / 15 / 10 / \\
\text { CCNY (1), } \\
\text { OK State (1), } \\
\text { PSU (1), } \\
\text { Rowan (1), } \\
\text { SMU (1) }\end{array}$ & $\begin{array}{l}\text { 2/0/1/1/ } \\
\text { Maine (1) }\end{array}$ & $\begin{array}{c}\text { 6/5/15/3/ } \\
\text { CCNY (1), } \\
\text { Columbia (1), } \\
\text { Drexel (1), } \\
\text { PSU (2), } \\
\text { SCU (2), } \\
\text { UWYO (1) }\end{array}$ & $\begin{array}{l}2 / 11 / 12 / 13 / \\
\text { CCNY (1) }\end{array}$ \\
\hline
\end{tabular}

\subsection{Design of Study}

Engineering students were solicited to participate in the ISES study, referred to publicly as the Engineering Pathways Study, at the aforementioned universities initially. It was clear that regardless of the compensation incentive, the study would need to be expanded to attract more students to come closer to meeting the approximate 400-student goal. The study was then expanded to Engineers Without Borders (EWB), which attracted students from over twenty universities across the country. This additional cohort of EWB students was thought to provide a form of extracurricular service learning (ESL) control for the study in addition to the students who were providing the no ESL control. Both control groups would provide perspective on students who are either already interested or not in participating in service learning. Approximately 250 students were recruited by the deadline of disseminating the first surveys and interviews. The volunteer engineering students were informed that this study is longitudinal in nature and were asked to participate for the full three years. Additional solicitations to the students asked for interest in thirty-minute phone interviews in exchange for additional compensation throughout the remainder of the study. From these solicitations students were selected for the interview process. Adaptations to the original survey and interview methods will be later in the chapter. As part of their participation, students are compensated for their time and efforts, which has additionally changed over the course of the three semesters thus far, increasing with student attrition in an effort to retain more students.

\subsection{Online Survey Results}

The aforementioned ISES survey students were asked to self-report scores on questions surrounding the idea of motivation (based on Carberry's work). These scores were 
synthesized down to one average score for motivation for each of semesters: Spring 2011, Fall 2011, and Spring 2012. Using Spring 2011 data, students were assigned categories of high or low based on whether they were above or below the population average (82.22). As students have a tendency to associate 0 to 100 scales to exams, quizzes and homework, striving for excellence causes some negative connotations with numbers even lower than 80 . As such, the nature of the respondents prompted the use of population average over other methodologies to categorize them into high or low motivation levels. An interest of what motivational changes occurred from Spring 2011 to Spring 2012 provoked further categorization. Four general tendencies categorized students into High-Decreasing, High-Static, Low-Static, and Low-Increasing categories. The categories were created using the standard deviation of the normalized change from Spring 2011 to Spring 2012 (Spring 2012-2011/2011). Those that were less than one standard deviation away were considered static and the rest were considered changing (increasing or decreasing depending on the starting point). These categories were assigned nominal values of $1=$ High-Static, $2=$ High-Decreasing, $3=$ Low-Increasing, and 4=Low-Static for the Multinomial Logistic Regression step, discussed below. The distribution of Spring 2011 to Spring 2012 scores for each category were tabulated and are depicted in Figure 3.1. Table 3.4 provides additional detail to better understand the background of each of the four cohorts. Figure 3.1 also helps to demonstrate changes for students, who initially start out in engineering programs, over a one-year period. As depicted, students predominantly fall in static categories over changing. As students progress through engineering, the hope would be that they become more motivated over time, yet over a one-year period this does not appear to be the case. The greatest potential for change is found in the L-I (Low-Increasing) category, yet it is one of the smallest. Whatever students are experiencing through their engineering experiences needs to be the catalyst for change in engineering design motivation. 


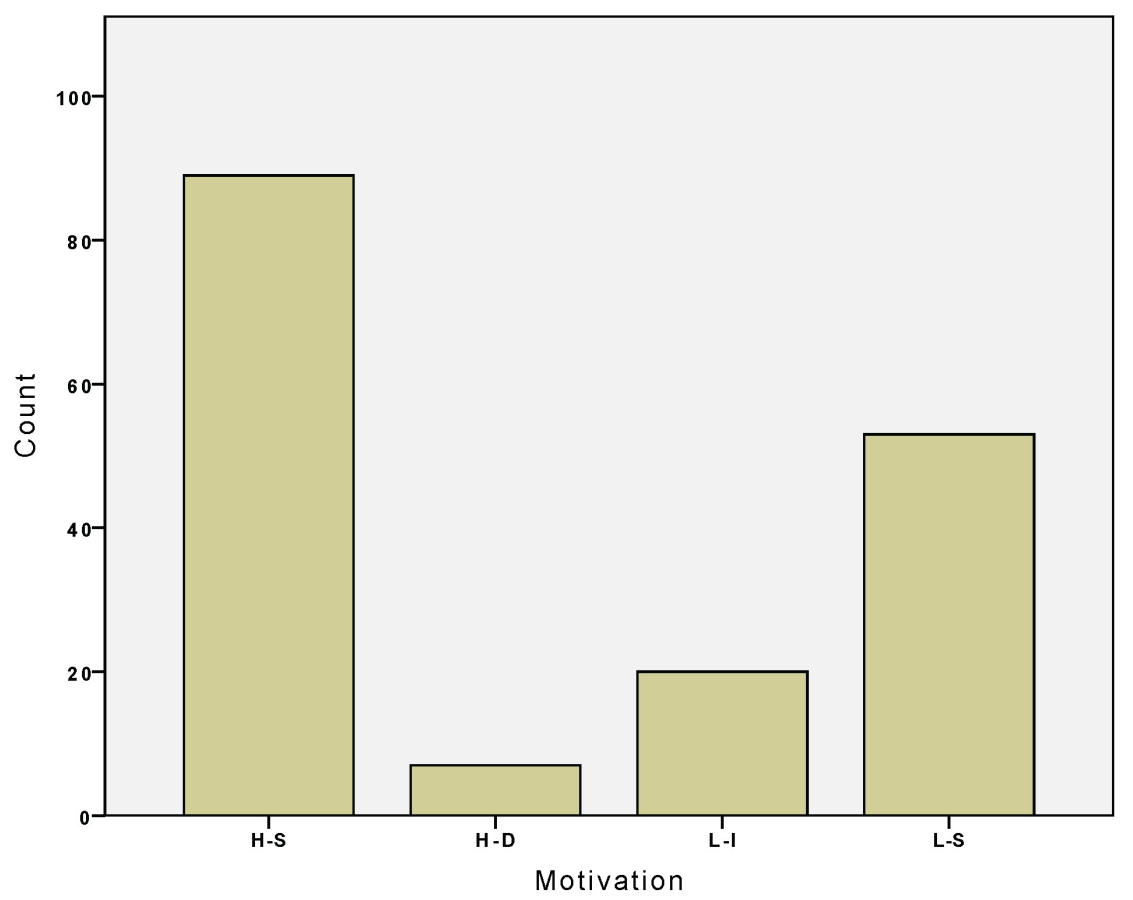

Figure 3.1: Spring 2011 to Spring 2012 motivation score distribution within H-S (High-Static), H-D (High-Decreasing), L-I (Low-Increasing), and L-S (Low-Static) categories.

Table 3.4

Interview "extreme" demographics

\begin{tabular}{|c|c|c|c|c|}
\hline & High-Static & $\begin{array}{c}\text { High- } \\
\text { Decreasing }\end{array}$ & $\begin{array}{c}\text { Low- } \\
\text { Increasing }\end{array}$ & Low-Static \\
\hline \# of Students & 10 & 10 & 10 & 10 \\
\hline \# Freshmen/Juniors & $1 / 9$ & $5 / 5$ & $6 / 4$ & $5 / 5$ \\
\hline$\%$ Female & $50 \%$ & $40 \%$ & $60 \%$ & $30 \%$ \\
\hline $\begin{array}{l}\text { Avg. GPA } \pm \\
\text { Standard deviation }\end{array}$ & $3.55 \pm 0.31$ & $3.45 \pm 0.45$ & $3.50 \pm 0.49$ & $3.60 \pm 0.32$ \\
\hline $\begin{array}{l}\text { Major: } \\
\text { (BE/CE/ChE/EE/ } \\
\text { EnE/ME/Other) }\end{array}$ & $0 / 1 / 0 / 0 / 1 / 4 / 3$ & $2 / 2 / 1 / 2 / 1 / 1 / 1$ & $0 / 2 / 1 / 0 / 3 / 2$ & $1 / 1 / 0 / 1 / 1 / 2 / 4$ \\
\hline $\begin{array}{l}\text { University: } \\
\text { (CU/JMU/MTU/ } \\
\text { Tufts/Others) }\end{array}$ & $\begin{array}{r}1 / 3 / 2 / 2 / \\
\operatorname{SCU}(1), \\
\text { Rowan (1) }\end{array}$ & $\begin{array}{l}0 / 1 / 3 / 2 / \\
\text { PSU (1), } \\
\text { CCNY(3) }\end{array}$ & $\begin{array}{c}\text { 1/2/4/1/ } \\
\text { Columbia(1), } \\
\text { Maine(1) }\end{array}$ & $\begin{array}{l}0 / 2 / 2 / 4 / \\
\operatorname{PSU}(1), \\
\operatorname{SCU}(1)\end{array}$ \\
\hline
\end{tabular}

\subsubsection{Logistic Regression}

The multinomial nature of the dependent variable suggested a logistic regression to be the best option for analyzing the survey data. Instead of running one large multinomial logistic regression and missing significant variables, the process was broken up into 
separate pieces using SPSS ${ }^{\circledR}$. This method took portions of the independent variables at a time and used them within a smaller multinomial logistic regression. This was deemed a more appropriate means of identifying statistically significant variables (with $95 \%$ confidence) when married with the dependent variable, since using all at once failed to yield any with statistical significance. From the segmented logistic regressions, those with statistical significance (with 95\% confidence) were set aside and used in one final multinomial logistic regression. Two logistic regression models were created utilizing two separate reference categories: High-Static and Low-Static which were selected to ensure all dimensions of the model could be observed. This method, however, created two separate models entirely since the statistically significant independent variables were selected for the final model (Appendix 11 depicts the detailed results).

Due to the factor analysis, some of the independent values in the logistic regression are questions and demographics, while some are the factors that resulted from previous reduction. The results are examined for each reference category primarily and secondarily for the comparative grouping for each of the questions, factors, or demographic. Due to the extensive dimensionality caused by the large quantity of independent variables, only those that were determined to be significant from both multinomial logistic regressions are presented in Table 3.5; the remainder are available by reference category in Appendix 11, highlighting those that are significant, and replicating those in Table 3.5. 
Table 3.5

Logistic regression significant independent values table

\begin{tabular}{|c|c|c|c|c|c|}
\hline $\begin{array}{l}\text { IV } \\
\text { Code }\end{array}$ & IV Description & Reference & Beta & Significance & Target \\
\hline ESM & \multirow{5}{*}{ Success Mean } & L-S & 1.072 & 0.005 & $\mathrm{H}-\mathrm{S}$ \\
\hline ESM & & L-S & 0.957 & 0.063 & $\begin{array}{l}\text { L-I } \\
\end{array}$ \\
\hline ESM & & $\mathrm{H}-\mathrm{S}$ & 0.953 & 0.026 & L-S \\
\hline ESM & & $\mathrm{H}-\mathrm{S}$ & 0.943 & 0.086 & H-D \\
\hline ESM & & $\mathrm{H}-\mathrm{S}$ & 0.921 & 0.003 & $\begin{array}{l}\text { L-I } \\
\end{array}$ \\
\hline$\overline{\text { EAM }}$ & Anxiety Mean & L-S & 1.051 & 0.072 & H-D \\
\hline INC.2 & \multirow{2}{*}{$\begin{array}{l}\text { Incentive to participate: } \\
\text { "Help society or solve a } \\
\text { societal need" }\end{array}$} & L-S & 1.056 & 0.029 & $\mathrm{H}-\mathrm{S}$ \\
\hline INC.2 & & $\mathrm{H}-\mathrm{S}$ & 0.951 & 0.021 & L-S \\
\hline INC.4 & \multirow[t]{2}{*}{$\begin{array}{l}\text { Incentive to participate: } \\
\text { "Learn something new } \\
\text { and/or gain experience" }\end{array}$} & $\mathrm{H}-\mathrm{S}$ & 0.947 & 0.039 & L-S \\
\hline INC.4 & & $\mathrm{H}-\mathrm{S}$ & 0.887 & 0.003 & L-I \\
\hline INC.7 & $\begin{array}{l}\text { Incentive to participate: } \\
\text { "Recognition" }\end{array}$ & L-S & 0.954 & 0.034 & $\mathrm{H}-\mathrm{S}$ \\
\hline SUC.1 & $\begin{array}{l}\text { Success at solving } \\
\text { engineering problems: } \\
\text { "My ability or skill"" }\end{array}$ & $\mathrm{H}-\mathrm{S}$ & 0.906 & 0.015 & L-I \\
\hline SUC.5 & $\begin{array}{l}\text { Success at solving } \\
\text { engineering problems: } \\
\text { "Support given to me by } \\
\text { my faculty, experts, or } \\
\text { team members" }\end{array}$ & $\mathrm{H}-\mathrm{S}$ & 1.041 & 0.081 & L-S \\
\hline SUC.6 & \multirow{3}{*}{$\begin{array}{l}\text { Success at solving } \\
\text { engineering problems: } \\
\text { and "The available } \\
\text { resources (i.e. materials } \\
\text { or facilities)" }\end{array}$} & L-S & 1.142 & 0.093 & $\mathrm{H}-\mathrm{D}$ \\
\hline SUC.6 & & L-S & 1.051 & 0.092 & $\mathrm{H}-\mathrm{S}$ \\
\hline SUC.6 & & $\mathrm{H}-\mathrm{S}$ & 0.942 & 0.031 & L-S \\
\hline $\begin{array}{l}\text { AF1 } \\
\text { WAV }\end{array}$ & Work Avoidance & $\mathrm{H}-\mathrm{S}$ & 2.009 & 0.085 & H-D \\
\hline $\begin{array}{l}\text { AF1 } \\
\text { MAP }\end{array}$ & Mastery Approach & L-S & 2.151 & 0.031 & $\mathrm{H}-\mathrm{S}$ \\
\hline $\begin{array}{l}\text { KSQ1 } \\
\text { F1 }\end{array}$ & Personal Strengths & L-S & 2.991 & 0.035 & L-I \\
\hline $\begin{array}{c}\text { KSQ1 } \\
\text { F4 }\end{array}$ & Future Plans & L-S & 2.224 & 0.023 & $\mathrm{H}-\mathrm{S}$ \\
\hline
\end{tabular}




\subsubsection{Success and Anxiety Mean}

The scores that successful or anxious students would feel in performing engineering design tasks resulted from nine similar questions to the engineering design motivation score, previously discussed, and were tabulated in a similar manner. These resulted in one overall mean score from nine Likert-type scale questions, from 0 to 100. These scores were used as independent variables within the logistic regression. Table 3.5 depicts with $90-95 \%$ confidence that as the Success mean increases, students are more likely to fall in the reference category than the Dependent Variable (DV) category with one exception: for the reference category, Low-Static students are more likely to be in High-Static than Low-Static with 95\% confidence. It was also determined that as these scores increased with Low-Static as a reference, students are more likely to be in the Low-Static group than the Low-Increasing (Beta: 0.957 times more likely with $90 \%$ confidence). With respect to the anxiety mean, students were asked to self-report their degree of anxiety in performing tasks on a 0 to 100 Likert-type scale. Table 3.5 shows students are more likely to fall into the High-Decreasing group than the Low-Static, with 90\% confidence (Beta: 1.051 times more likely).

These results help to support that those with an increased level of anxiety with respect to engineering design tasks are more likely to have lower level of motivation. As the logistic regression help to demonstrate, students are more likely to fall in the HighDecreasing group than the Low-Static group, suggesting they are becoming less motivated rather than staying the same or increasing. Alternatively, those who have a higher level of success should be more motivated to perform engineering design tasks due to the positive feelings that come with success. The results of the logistic regression show, with only one exception, that as success levels increase, students are more likely to be in higher levels of motivation or are more likely to stay highly motivated than to decrease in motivation. Both help to support the hypothesis related to students' success.

\subsubsection{Incentive to Participate}

Three questions from an instrument within the survey which asked students to rate choices on the degree the choice would impact their incentive to participate and complete an engineering design task were found to be statistically significant, with $95 \%$ confidence. For the "Help society or solve a societal need" and "Learn something new and/or gain experience" choices it was found with one exception that students were more likely to fall into the reference category than the DV category, with $95 \%$ confidence. However, for students' incentive to participate with the "Help society or solve a societal need" task in mind they are more likely to be in the High-Static than the Low-Static category.

Results of the logistic regression help to confirm the satisfaction hypothesis previously outlined. The argument that if students' incentive to participate in engineering design tasks increases, their motivation to participate should also increase. Further examining the questions utilized in the logistic regression with only one exception, all of the questions support this claim. The results help demonstrate that as students' incentive to 
participate in engineering design tasks increases, they are more likely to be in the highly motivated category over the low. The exception pertains to students' incentive to participate with regard to recognition of engineering design tasks, which is the students' ability to identify what constitutes an engineering design task; these students are more likely to be in the low category than the high category of engineering design motivation. Preliminary responses to this exception suggest that students may dislike or struggle with recognition of engineering design tasks. Future analysis will help to determine if this continues to be an exception for participating students and help to determine why this is the case.

\subsubsection{Impact on Success}

Additionally, students were asked to rate their degree the choice would impact whether they were successful or unsuccessful in solving an engineering problem on a 0 to 100 scale. For three out of the five instances where questions within this instrument were deemed statistically significant, students were more likely to be in the target category than the reference category (Table 3.5). However, in one instance for students' selfreported degree of how much "My ability or skill" and/or "The available resources (i.e. materials or facilities)" would impact their success, they were more likely to be in the reference category. For these two specific aspects, this suggests that students are more likely to be in the high motivation category.

The logistic regression helps to support the claim that as scores for tasks associated with impacting success increases, students' engineering design motivation also increases. This additionally helps to support the hypothesis pertaining to satisfaction, previously outlined. Satisfaction applied to engineering design motivation is being described as students' perceived feelings of success combined with incentive to complete engineering design work. It's suggested that if students' perception of how tasks will impact their level of success are increasing, their motivation to perform those tasks will also increase. It was found that as the scores associated with success increased, students were more likely to fall in the high category of motivation than the low. However, students reported the degree "Support given to me by my faculty, experts, or team members" would impact whether they were successful or unsuccessful would more likely place them in a low motivation than a high motivation category. This could be attributed to the magnitude of influence faculty, experts and team members have on the feeling of success. This could provide grounds for universities to ensure students are getting the help they need to improve overall success and motivation.

\subsubsection{Attitudes}

Factor analysis resulted in five factors for the Attitudes instrument within the survey. In the process of validating this instrument, factor analysis was utilized to reduce the independent variables down to a smaller set. This same process was utilized in validating the instrument for the ISES study. It resulted in the same five factors; Mastery Approach, Mastery Avoidance, Performance Approach, Performance Avoidance, and Work Avoidance. Of the two factors that were found to be statistically significant within the logistic regression, both Work Avoidance and Mastery Approach suggest students would be placed in the DV over the reference category. 
The results of the logistic regression help claim that higher levels associated to mastery and performance approach attitudes will promote higher levels of motivation to do engineering design work than those with higher levels of avoidance attitudes. The assumption associated with students who tend to avoid work is that they are more likely to have lower levels of motivation, whereas those who strive to master the work they are given are more likely to have higher levels of motivation. As previously discussed, attitudes questions were reduced to factors. The results of the regression support the claim that as levels of mastery approach increase, students are more likely to be in the high motivation group than the low. Additionally, it also supports that as levels of work avoidance increases, students are more likely to be decreasing in motivation than staying the same; they are more likely to be in High-Decreasing than High-Static. Future work will continue to examine the effects of performance to see if it becomes a significant factor in motivation to do engineering design tasks.

\subsubsection{Knowledge and Skills}

Lastly, within the Knowledge and Skills instrument inside the online survey students were asked to answer a series of fifty-one questions. Factor analysis was also used in reducing the number of independent variables within this instrument down to nine factors, however, only for the purposes of the ISES study (Appendix 9.1 and 9.2). These factors include: (1) recognizing and evaluating personal strengths (Personal strengths), (2) communication and teamwork skills (Teaming),(3) indicators of personal improvement (Improvement), (4) Future plans, (5) engineering mindset (Process recognition), (6) Design process, (7) engineering technical skills (Professional practice), (8) holistic thinking (Impact assessment), and (9) project management (Planning). Of those factors, two appear to be statistically significant and suggest students are more likely to fall in their target than the reference category with $95 \%$ confidence.

The results of the logistic regression help to support the claim that a greater level of knowledge and skills provides a basis for which students increase their motivation as a result of participating in engineering design programs. It is suggested that a greater level of knowledge and skills helps to support higher levels of motivation. With regard to one identified factor, Personal Strengths, as the level increases, students are more likely to have higher motivation. The Personal Strengths factor resulted from six tasks associated with knowledge and skills students were able to attain through university experiences (i.e. set and pursue my own learning goals, apply interpersonal skills in managing people). This suggests that personal strengths contribute to engineering design motivation. Additionally, another factor, Future Plans, reveal a positive correlation with motivation. This also suggests that future plans contribute to overall student motivation to perform engineering design tasks.

\subsection{Interview Results}

As previously mentioned, interview materials provide in-depth information enabling some observational studies to be conducted in addition to the quantitative analysis. The aforementioned forty out of the 121 interview participants were evaluated for the purposes of the motivational analysis. From the Spring 2011, scores the top twenty and 
bottom twenty scores for student motivation were selected as the "extreme" students having high or low motivation to start with for the study. Next, the change in score was calculated from Spring 2011 to Spring 2012 and normalized to Spring 2011 (Spring 2012-2011/2011). From the high and low clusters, ten students were selected for each group based on their relative closeness to zero. These students will further be referred to as stable/static, with very little change over time if any. Additionally ten students were selected with larger differences from Spring 2011 to Spring 2012 and were deemed as decreasing or increasing categories. Students who did not complete the Fall 2011 survey were excluded from these extreme categories for future analysis limitations. In addition to the change in motivation scores over time, students within this group may have also changed majors (including those who are no longer in engineering). Motivations for all students within the four "extreme" groups were tabulated. Table 3.4 helps to further analyze these four categories of "extremes," demonstrating the diversity in participants, as well as the similarities. For example, GPA gives the perspective of how all categories appear to maintain relatively high grades despite their year or level of engineering design motivation. This aids in further understanding how comparisons can be made in addition to their change in motivation to do engineering design tasks from Spring 2011 to Spring 2012.

In addition to examining the "extremes" within the interview pool survey data to determine their increasing, decreasing, or static status, data from the interview text was also examined. As previously discussed, the interview text was coded using HyperResearch ${ }^{\circledR}$ using in vivo thematic coding. These codes were also tabulated using the software frequency reporting option. Focusing on the extremes, thirty codes were created for 120 interviews (from forty individuals for all three semesters). Appendix 12 further illustrates the codes and their meanings. Results of the frequency reports, as well as what the information has been used for thus far can be found in the results section of this chapter.

The "extreme" interview participants ( $\mathrm{n}=40$ per semester for a total of 120$)$ were evaluated utilizing the frequency report option in HyperResearch ${ }^{\circledR}$. These perspectives are meant to be a representative sample of the entire ISES participant pool, as well as for engineering students as a whole. The frequency report option enabled the data to be tabulated for a better understanding of student motivations and help determine if education or gender helps to influence motivation to do engineering design tasks. A listing of codes can be found in Appendix 12. Of the thirty-five code tags created, approximately thirty were used in the coding process. Some of these codes were from first iterations and were later discarded for various reasons (i.e. better descriptions were realized, condensed versions were created, etc.). Tables 3.6, 3.7, and 3.8 depict the top five motivations for all "extreme" students, a gender breakdown, and a class rank breakdown, respectively.

\subsubsection{All Participants}

Generally the top motivations for participants falls into a more inwardly focused genre; topics like personal Interest/Enjoyment, whereas the remaining motivations are more pragmatically focused personal drivers. Many students remarked that their interest in the 
community service topic or experience was what motivated them to continue doing engineering design work. For example, a High-Static student from Fall 2011 noted:

The one thing that comes to mind is the projects and subject matter we actually work on it just interests me the most you get to design projects and actually get our hands dirty circling things that's what I want to do so keeps me interested.

There also seemed to be a common trend of commenting on circumstances, which enabled them to apply the knowledge students were gaining through their engineering curriculum, as well as just gaining knowledge in general. A High-Static student in Fall 2011 commented on the opportunity to apply their knowledge and skills:

[I] think the best part about it is that it's a lot of math and you can actually use math for something that seems...realistic and worthwhile.

Additionally, a Low-Static student commented on the future applications of the knowledge they were gaining through coursework:

The possible-or the knowledge that... what I'm doing will be... what I'm going to be learning in the future.

Although these first impressions provide some initial insight on engineering students (along with those students who have switched out of engineering), further work, as well as the remainder of this chapter will provide additional insights on engineering student motivations.

Table 3.6

Top five motivations expressed by all "Extreme" interview participants (n=120); rank (frequency), $n=1035$ code tags from all participants

\begin{tabular}{|l|c|}
\multicolumn{1}{|c|}{ Motivation } & $\begin{array}{c}\text { Rank } \\
\text { (frequency) }\end{array}$ \\
\hline Interest/Enjoyment & $1(14 \%)$ \\
\hline Application of Knowledge and Skills & $2(11 \%)$ \\
\hline Knowledge & $3(9 \%)$ \\
\hline Personal Significance & $3(9 \%)$ \\
\hline New Perspective & $4(7 \%)$ \\
\hline Real World Experience & $4(7 \%)$ \\
\hline Future Circumstances & $5(6 \%)$ \\
\hline New Experience & $5(6 \%)$ \\
\hline
\end{tabular}




\subsubsection{Gender Influence}

Engineering tends to be predominantly comprised of white males (Jordan et al. 2011; NAE 2008; Besterfield-Scare et al. 2001). In order to better understand how to retain females in a male dominated field a better appreciation of what motivates females to participate is a necessity. Table 3.7 demonstrates a representation of the top motivators broken down by gender, which additionally helps to address whether female students are more motivated to participate in engineering design programs when given the opportunity to apply what they are learning to help others. Although the first three reasons are similar (accounting for 36\% for males and 33\% for females), the remaining motivation codes begin to shed some light on why females are pursuing the engineering field. Females ranked new experience, future circumstances, and personal significance higher than male participants. For example, a High-Decreasing student from Spring 2012 commented on a new experience inspiring her to continue pursuing engineering work:

Last December I went on the 1st assessment trip to Bouma for the water project and...I met amazing people that were really inspiring... and took a lot of really cool water quality data that... was kind of fun and yeah I mean just seeing the village come together and they all want the same thing they all want clean water and they were they were they had like the hope that we could help them and they think we inspired them to do more... in the village too so that was kind of good.

Additionally a Low-Increasing student from Fall 2011 commenting on her future goals and how an opportunity helped to motivate her:

I am looking to work in international development... and so it's been nice to get a start on that...while I'm still in undergraduate.

These quotes help to support hypotheses associated with the logistic regression results pertaining to knowledge and skills' future circumstances factor, as well as the satisfaction related factor: the incentive to do engineering design work for the purposes of helping others. Although these are simply different reasons why males and females believe they joined their respective engineering program, they provide a perspective for what specifically provoked them to pursue engineering curriculums. These insights provide a basis for which universities can begin to attract and further retain female engineering students. For example, when universities are recruiting students they could suggest opportunities for new experiences and relate them to career choices, addressing two motivating factors for females. When these female students consider their program and university options those that highlight specifics related to their interests and motivations are going to be higher on their list than those that do not. Although this initial examination of the interview results cannot conclusively suggest that females are more motivated than males, Figure 3.2 does provide some preliminary insight on average engineering design motivation differences between males and females. As this study continues and participants progress through their respective curriculums these 
preliminary generalities can be re-evaluated to determine if they hold true over time with the same individuals, to provide a more in depth insight on new and incoming students.

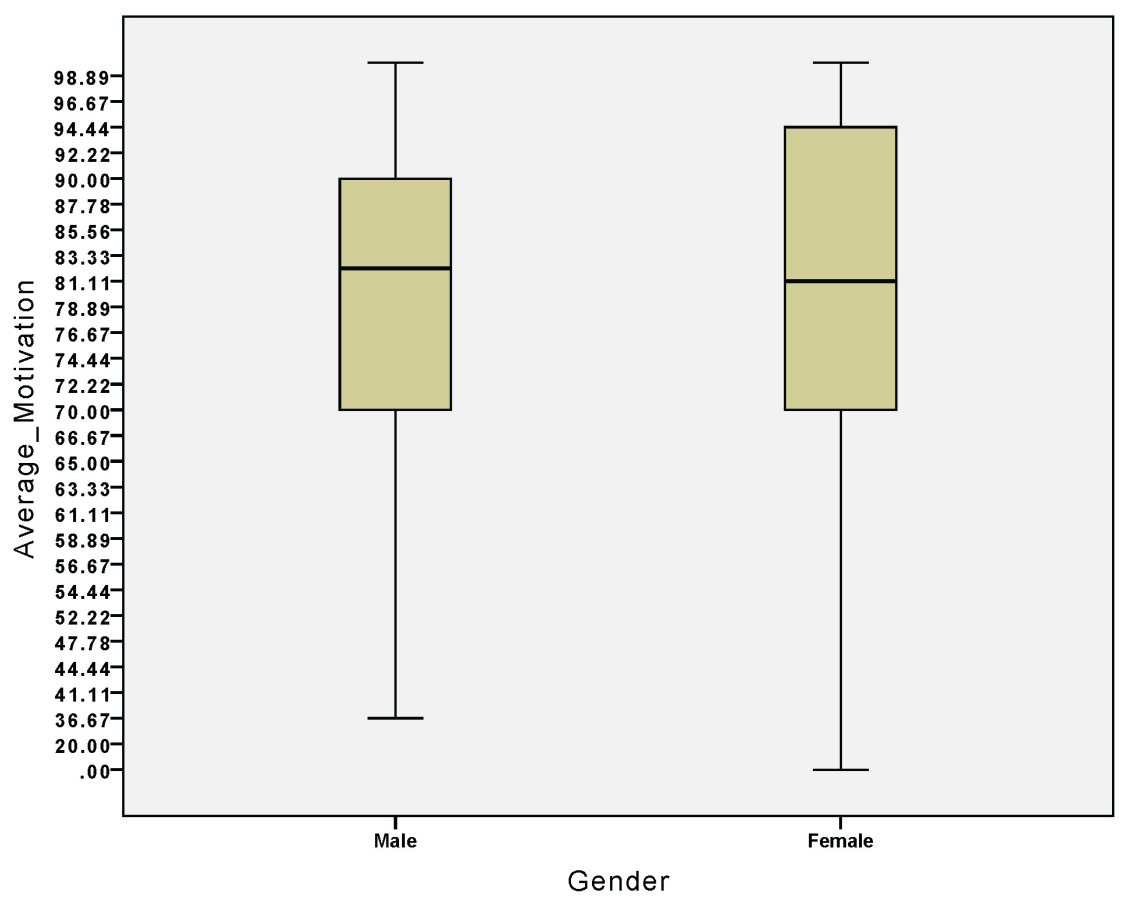

Figure 3.2: Spring 2011 average motivation score distribution by gender.

Table 3.7

Top five motivations expressed by men and women ( $n=54$ and 36, respectively) "Extreme" interview participants; rank (frequency), $n=572$ and 463 , respectively, code tags for all three semesters

\begin{tabular}{|l|c|c|}
\hline \multicolumn{1}{|c|}{ Motivation } & $\begin{array}{c}\text { Male Rank } \\
\text { (frequency) }\end{array}$ & $\begin{array}{c}\text { Female Rank } \\
\text { (frequency) }\end{array}$ \\
\hline Interest/Enjoyment & $1(15 \%)$ & $1(13 \%)$ \\
\hline Application of Knowledge and Skills & $2(12 \%)$ & $2(11 \%)$ \\
\hline Knowledge & $3(9 \%)$ & $3(9 \%)$ \\
\hline Personal Significance & $4(8 \%)$ & $3(9 \%)$ \\
\hline Real World Experience & $5(7 \%)$ & $5(6 \%)$ \\
\hline New Perspective & $5(7 \%)$ & $5(6 \%)$ \\
\hline New Experience & & $4(7 \%)$ \\
\hline Future Circumstances & & $5(6 \%)$ \\
\hline
\end{tabular}

\subsubsection{Academic Level Influence}

In addition to evaluating gender difference, an interest in what students gain from having additional education has prompted the examination of their respective motivators. As previously mentioned, it is thought that students with more years of education are more motivated to do engineering design. Observations of interview responses help to support or disprove whether this claim can be made. Table 3.8 depicts the breakdown of the top five motional codes for freshmen and junior participants. Similar to previous 
HyperResearch ${ }^{\circledR}$ frequency results, the top three motivations are students' interest/enjoyment in the topic or experience, their interest in gaining knowledge, as well as applying their knowledge and skills, accounting for 36\% for freshmen and 34\% for juniors. For example, a High-Decreasing student in Spring 2012 commented on interest motivating them to do engineering design work:

I'm really enjoying my statics class...honestly it's easier than physics...or any or chemistry basic chemistry classes but it's like actually putting engineering...like thought into place.

Despite these similarities, the differences can be seen in the breadth of motivations in participants having more education. This could be attributed to participants learning more about their field and finding there is a lot more contributing to why they are sticking with their program. Conversely, freshmen have had limited time in which to determine why they are motivated to complete tasks or participate in experiences. As previously stated, these are some preliminary generalities and future work will help to determine if this continues to be the case for these participants over time.

Table 3.8

Top five motivations expressed by freshmen and juniors ( $=51$ and 69, respectively) "Extreme" interview participants; rank (frequency), $n=442$ and 593, respectively, code for all three semesters

\begin{tabular}{|l|c|c|}
\hline \multicolumn{1}{|c}{ Motivation } & $\begin{array}{c}\text { Freshmen Rank } \\
\text { (frequency) }\end{array}$ & $\begin{array}{c}\text { Junior Rank } \\
\text { (frequency) }\end{array}$ \\
\hline Interest/Enjoyment & $1(15 \%)$ & $1(13 \%)$ \\
\hline Knowledge & $2(11 \%)$ & $2(8 \%)$ \\
\hline Application of Knowledge and Skills & $3(10 \%)$ & $1(13 \%)$ \\
\hline Personal Significance & $4(9 \%)$ & $2(8 \%)$ \\
\hline Future Circumstances & $5(7 \%)$ & $4(6 \%)$ \\
\hline Real World Experience & & $2(8 \%)$ \\
\hline New Perspective & & $3(7 \%)$ \\
\hline New Experience & & $4(6 \%)$ \\
\hline Friends & & $5(4 \%)$ \\
\hline
\end{tabular}

\subsection{Conclusions}

This chapter aimed to address several varying hypotheses using quantitative and quantitative methods; all working to determine what motivates students to do engineering design tasks. The discussion and conclusions of the results of the quantitative and qualitative methods are found below.

This process attempted to determine whether greater female involvement in the study resulted in higher levels of motivation. Upon completion of the logistic regression it is clear to say that at this point these hypotheses cannot be proven within this set of data. Gender [female] did not have a significant impact on the level of motivation (Appendix 11). Further data collection as this study continues as well as examining this hypothesis 
with a different set of data entirely may help to prove otherwise. Additionally, the hypothesis was examined using qualitative methods from a more ethnographic approach within the interview data. The findings conclude that females are motivated by interest/enjoyment, application of knowledge and skills, knowledge, as well as several other factors, however, males are similarly motivated by these factors. Claims cannot be made to suggest that females are more motivated than males, yet this information can still be utilized in attracting females to engineering programs. Instead of generally recruiting students, these motivating factors can be specifically pointed out to generate interest from future students, as well as from current students. Maintaining interest by motivating students and reminding them why they want to participate in engineering programs may help to retain and attract additional students who may have otherwise opted out.

Additionally this study attempted to address whether a greater level of knowledge and skills provides a basis for which students increase their motivation as a result of participating in engineering design programs. The logistic regression helped to prove that this is true through the use of two factors: Personal Strengths and Future Plans (Appendix 11). Additionally, the interview responses help to support these factors, one Low-Static from Spring 2012 commented on future circumstances motivating them to continue to do engineering design work:

\section{The best part about a major [is] the different options I'm going to have when I graduate.}

These students are more likely to be increasing their motivation or to be in the higher motivation category than to be in the consistently low group. Similar to gender, as this study continues further analysis of an increasing population size may help to continue to prove this hypothesis true.

Next the hypothesis of whether higher levels of students' perception of their mastery and performance approach attitudes will have higher levels of motivation to do engineering design work then those with higher levels of avoidance attitudes was examined. This process utilized the five factors resulting from factor analysis of the ISES study data, as well as from the factor analysis conducted to validate the instrument initially. Although performance approach was determined to be inconclusive at this time, mastery approach was useful in providing support for this hypothesis. It was found for students in the ISES study that as their levels of mastery approach increased, they were more likely to be in the high motivation group than the low. Alternatively, when examining work avoidance it was found that as the levels increased students were more likely to be decreasing in motivation than to remain the same in the highly motivated category. Both of these findings help to support that levels of mastery approach attitudes will have higher levels of motivation than those of avoidance attitudes.

The hypothesis suggesting that a greater sense of personal satisfaction associated with students' self-reported level of success and incentive to complete engineering design 
tasks leads to higher motivation was also evaluated. This hypothesis was examined using two sets of questions, pertaining to students incentive to participate, as well as their degree of feeling successful/unsuccessful with engineering design tasks. The claim that as these levels increased students should be more motivated to complete engineering design tasks was supported with only one exception for each set of the statistically significant questions. For both question pertaining to incentive, as well as those pertaining to level of success students were more likely to be in the high motivation category than low. The exception for student incentive was related to recognition of engineering design tasks; students were more likely to be in the low motivation category than the high. Future work could be done to focus on trying to motivate students using recognition of engineering tasks as a tool. As students feel more comfortable with this ability, they may be more motivated by it. Additionally, support from faculty, experts or team members was noted as something that would likely place students in a low motivation group over a high. Working to ensure students have good peer experiences, as well as having faculty who are supportive and available to help, could help to turn this question around for student motivation. Further analyses will work to examine these questions to determine if students' scores change over time as well as if there are any additional exceptions and what might be causing them.

Next, self-reported scores for success, as well as for anxiety were explored using similar means to the motivational scores associated with engineering design tasks. The logistic regression demonstrated that for an increase in mean of success students are generally more likely to be in the High-Static group than the other categories based on multiple results of significance indicators in the multivariate logistic regressions with both reference categories. This helps to support the hypothesis associated with success, suggesting that as this score increases students are more motivated. Conversely, the claim that as anxiety scores increase motivation to do engineering design tasks should decrease was also supported. The logistic regression help to demonstrate that as the anxiety level increased students were more likely to be in the High-Decreasing group than the Low-Static. This helps to suggest that students actually are losing motivation as their anxiety levels increase. Further analysis will continue to evaluate these score to determine if this is consistent over time.

Lastly, using qualitative ethnographic approaches, the hypothesis related to level of education was evaluated to determine if more educated students are more motivated to do engineering design tasks. Although these observations cannot provide statistical confirmation, they can provide some initial insights. Examining ISES students, juniors resulted in more motivating factors than freshmen, which helps to support the claim that these students are more motivated by a variety of factors. This cannot prove, however, that juniors were more motivated than freshmen were to perform engineering design tasks. Future evaluations will help to reaffirm these preliminary observations and work to statistically prove the aforementioned hypothesis.

These results provide some preliminary insights about where students place themselves based on various independent variables and can suggest where to focus efforts to better 
motivate engineering students to perform design tasks. The additional examination of the detail-rich information embedded within the interview responses suggests focusing on appealing to student interest/enjoyment, the application of knowledge and skills, as well as assuring the amount and kind of knowledge they can obtain as a result of participating in engineering design tasks.

\subsection{Next Steps}

Due to this study being longitudinal in nature, data collection will continue for another three semesters. Additional analysis will give further insights on engineering curriculums based on student responses from across the board. Some better understandings of how to retain and attract females could also result from further analyses. Responses within the interviews and surveys could provide a means with which to alter curriculums to better personalize and meet the needs of current, incoming and prospective students. 


\section{4. idesign}

This chapter primarily gives a narrowed focus on one specific LTS academic program, which demonstrates the use of instruments serving as an example for a more formalized assessment. Later chapters will examine broader studies as well as some takeaway generalities. Some of the preliminary results of the utilized assessment instruments (outlined in Chapter 2) are provided to begin examining what can be done with this kind of program. Although the formalized assessment provides layers of information, which can be used in various ways, concentration will be given to the details pertaining to motivation.

\subsection{Background}

The idesign program is an international senior design program through Michigan Technological University. Within this program, students from various engineering majors participate in pre-fieldwork preparation. The fieldwork component is completed over the course of two weeks, currently in either rural Panama or Ecuador. Upon returning to Michigan Tech, students participate in a semester long design course where they compile their collected data from their field site and work to come up with a suitable design to solve the posed problem while in country. Prior to participation, students are also asked to complete a motivational narrative describing why they wanted to participate in the program, along with some online survey questionnaires aiming to better understand their motivations. Once students have completed the design portion they are again asked to complete online survey questionnaires. This pre and post methodology aims to begin understanding the whole picture of the student experience, rather than just one point of the program. Embedded in these surveys (and narratives) are motivational questions. This section discusses some preliminary results of these surveys made possible by the present cohorts of students who completed their fieldwork and design work in the Summer and Fall of 2011, respectively.

\subsection{Program Description}

A key question for this program is: Why do students get involved in the first place; what motivates them? Due to the increasing interest in programs like these, an assessment was developed to begin gathering information from Michigan Tech students as a form of an internal assessment. This ongoing study hopes to utilize the information being collected to better understand the needs and motivations of student participants as part of a larger study of the stakeholders. This chapter analyzes this information in an attempt to address previously outlined hypotheses pertaining to gender distribution, readiness, intercultural competency, sustainable engineering knowledge, international experience, as well as education level influence on student motivations (hypotheses 1, and 3-7 outline in Chapter 1). The understanding of this data and the conclusions related to these hypotheses is meant to enhance the program for future classes and better understand why students are motivated to do engineering design work.

\subsection{Program Assessment}

Upon entering the program, students are asked to complete a series of surveys and questionnaires. These instruments include (see Appendix 7 for samples of these instruments): 
1) a Readiness assessment,

2) Intercultural development inventory (IDI),

3) a Skills and Attitudes assessment,

4) a Sustainable Engineering survey (SESL)),

5) an Impacts of Service on Engineering Students survey (ISES), and

6) a Motivational narrative

As the explaination in Chapter 2 demonstrates, these instruments are used to assess the program qualitatively and quantitatively (Table 4.1), and provide insights on what is motivating these students to do engineering design work.

Table 4.1

idesign assessment instruments

\begin{tabular}{|l|c|}
\hline \multicolumn{1}{|c|}{ Instruments } & idesign \\
\hline Readiness Assessment & $\mathrm{X}$ \\
\hline Intercultural Development Inventory & $\mathrm{X}$ \\
\hline ISES (Motivation) & $\mathrm{X}$ \\
\hline SESL & $\mathrm{X}$ \\
\hline Word Cloud & $\mathrm{X}$ \\
\hline Motivational Narratives & $\mathrm{X}$ \\
\hline Interview Protocol & \\
\hline
\end{tabular}




\subsection{Design of Study}

Although this program is open to any major and discipline, students tend to be enrolled in engineering programs. The program starts with some initial meetings during which some of the instruments are given. For the purposes of this study, students who participated in these assessment methods were included and those who did not express interest and participate have been excluded from the program analysis. Admittedly, program provides an opportunity to examine the effects of short-term community engagement, as later described in Chapter 5. These students only spend two weeks in the country, while the remainder of the time is spent on campus designing and presenting their findings, thus narrowing the focus of students' experience. This limitation requires students to plan what they are going to do while in country ahead of time to ensure they are able to collect all of the data they will need for the design-work semester. The time constraint similarly reflects real world project time lines and gives students an opportunity to practice in a professional-like setting prior to graduation. As this program continues to grow and attract more students, the opportunity for assessment changes may be made possible, but at this time the focus remains on the participating students.

\subsection{Quantitative Results}

Some of the general demographic information for the largest number of participants for a given method is provided in Table 4.3. Additionally a comparative breakdown of similar demographics is presented in Figure 4.1 to demonstrate the spread of the participants' background. The results of the instruments outlined in Table 4.1 are presented below, although all instruments vary in number of participants who completed them.

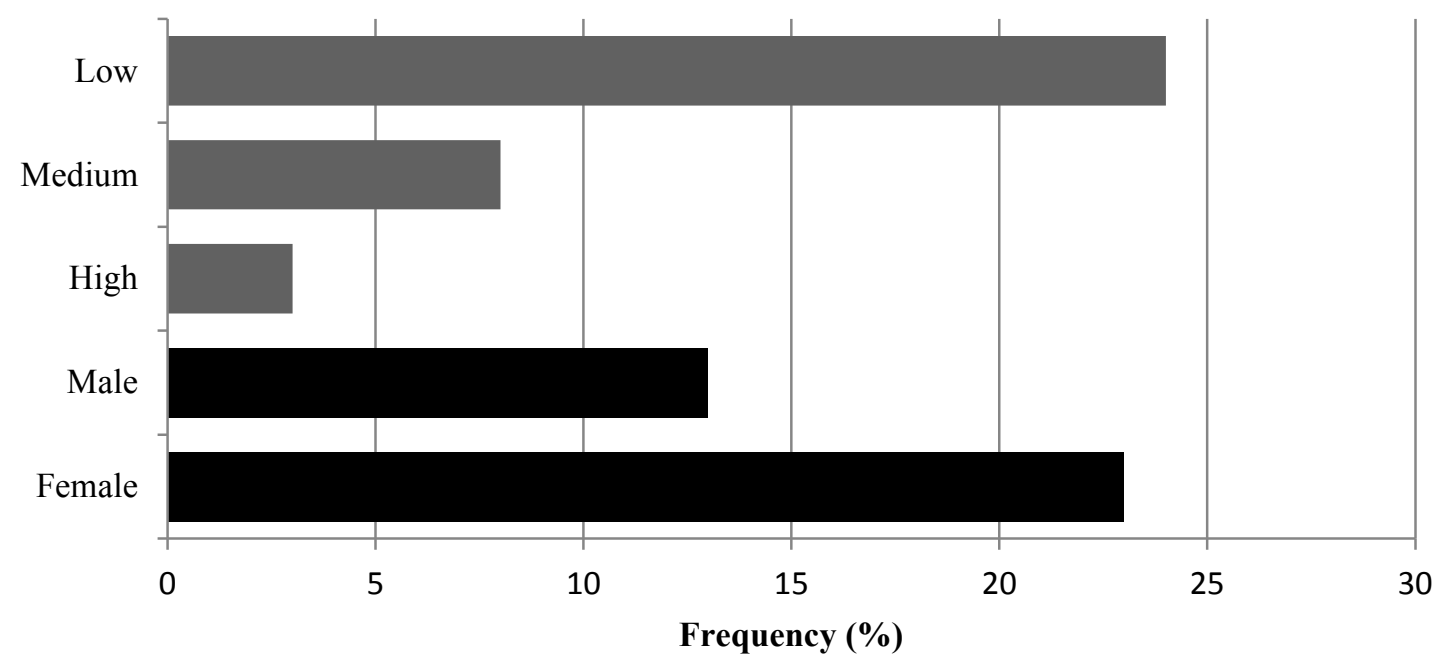

Figure 4.1: idesign student participant demographics in two categories: gender, and prior international experience $(n=36)$ 


\subsubsection{Readiness}

Students were asked to complete a Readiness to perform engineering design tasks ssessment in order to further evaluate their motivations for pursuing the idesign program as well as to look into what is motivating them to do engineering design work. The Readiness assessment is comprised of 20 Likert-scale (Strongly disagree $=-3$ to Strongly agree $=3$; zero was omitted). Included in the assessment were negatively phrased questions to eliminate bias in the data and ensure understanding of the questions being posed. These questions were tabulated and the results for idesign participants are displayed in Table 4.2 below. At this time the Readiness assessment has not been implemented for the post program experience, thus limiting the available data for analysis. Chapter 2 outlines the idea of a threshold for Readiness to perform engineering design tasks scores, which was utilized in preliminary observational analysis of the available scores. Initial idesign scores suggest that students are ready to participate in engineering design tasks, thus providing initial support for the claim that students with higher levels of readiness are more motivated with respect to engineering design tasks. This cannot be statistically confirmed at this time, however. Additionally Table 4.3 further analyzes the readiness scores looking at the gender breakdown. These results additionally help to support the claim that female students are more motivated to do engineering design tasks, as their scores are higher than males. Future work will utilize pre and post scores to determine if there is a statistically significant impact on engineering design motivation.

\subsubsection{Intercultural Competency}

Students' intercultural awareness was collected using the Intercultural Development Inventory (IDI) for the undergraduate (pre $n=55$, post $n=23$ ) student group ( see Chapter 2 for further details). The hope of measuring the IDI pre- and post- fieldwork would be to see some improvement to show that the students engaged in these programs are gaining a better understanding of how to work with someone who has a different worldview, culture, or life experiences and to shed some light on their motivations for participating in the program. A significant aspect of the idesign program is creating a solution for a community within the developing world. Having a better understanding of students' ability to work with individuals like the ones within their communities will provide insight on their motivation to do engineering design work in this capacity. Post scores for undergraduates are fulfilled upon the completion of their community projects, which differs from the graduate program later described in Chapter 5.

Table 4.2 depicts the scores for the undergraduate program, including those participants who did not complete the post experience IDI survey. An initial view of the table suggests the undergraduate average appears to decrease from pre to post international experience. While the reasons for this are less than certain, preliminary findings from post experience interviews suggest many students have broadened their worldview and realize there is a lot they have to learn. Lower scores might suggest the students are beginning to understand that they initially rated their understanding too high, a realization they had after their experience in country where they began to comprehend they didn't understand as much as they initially anticipated prior to fieldwork. 
Additionally the breakdown of the results by gender is depicted in Table 4.3, which demonstrates higher IDI scores for female participants. These results also help to support the claim that female participants are more motivated to do community-based engineering design work because of their understanding of cultural needs. Initial observational analysis of the scores help to demonstrate that idesign students are on average ten points lower than PCMI students (further detailed in Chapter 5), suggesting that IDI might have an impact on the level of engineering design motivation required for involvement in a given program. As the use of this instrument is continued, a better understanding of changes students are experiencing may become more apparent. Additionally, the use of this instrument might find some better correlations to student motivations, leading to further means of promoting and enhancing the program.

\subsubsection{ISES (Motivation)}

Of the four components within the survey mentioned in Chapters 2 and 3, the selfefficacy section focusing on motivation (Carberry et al 2010) was analyzed using the motivational scores as a dependent variable. Within the Engineering design self-efficacy section of the survey, students are asked to self-report scores for their motivation for nine specific engineering design questions. An average of these nine questions is taken to produce an overall motivation score with respect to engineering design, which serves as the aforementioned dependent variable. Within the idesign group of students, they will be placed into pre or post coursework categories, creating a dichotomous dependent variable; however, at this time only a pre score is available for initial observations. In order to best represent the provided information, a table was utilized. Table 4.2 below depicts the breakdown for the idesign program and Table 4.4 demonstrates the breakdown by gender. As previously detailed in Chapter 2, the threshold of 50 is utilized in this instance where a post score is unavailable. Some initial observations of this data reveal participants' average, as well as their minimum, are both over the threshold, suggesting these students are predominantly highly motivated to perform engineering design tasks. Although the breakdown of gender continues to support students being over the threshold value of 50, it does not demonstrate higher scores for women to support higher levels of engineering design motivation. As more information becomes available, further analyses and evaluations can be done to better understand the idesign participants. This information will also be used in similar scenarios to what Chapter 3 outlined.

\subsubsection{SESL}

Students' self-efficacy, beliefs, and interests related to sustainable engineering were assessed using an instrument known as SESL (Sustainable Engineering through Service Learning). Table 4.2 depicts the change in SESL score as a result of the preparation period for the idesign students. It is apparent that students are increasing their appreciation of sustainable engineering as a result of the coursework and/or group discussions. Additionally, the loss of students from pre to post experience is demonstrated in the Attrition column in Table 4.2. This helps to depict what kind of students are dropping out of the survey pool. For SESL, these students are predominantly female with relatively similar scores. Sustainable engineering is something the idesign program strives to incorporate into the program to ensure the 
longevity of any implemented design solution. It is thought that if students have a better understanding of sustainable engineering they will be better equipped to work on a project where the utilization of this skill is critical. Additionally, the claim is made that if students' have a better appreciation for aspects of a project they are working on, they will be more motivated to complete the task. Therefore the goal of evaluating students' self-efficacy, beliefs, and interests related to sustainable engineering is to link their understanding and ability to their motivation to participate in the idesign program.

An additional examination of the gender breakdown for SESL is demonstrated in Table 4.4, which for post and attrition scores helps to demonstrate females have higher scores. This helps to support the hypothesis that females are more motivated to do engineering design tasks as a result of having higher scores in sustainable engineering self-efficacy, beliefs, and interests. Although these preliminary findings are limited, future work may be able to look deeper into this information to determine if this is also increasing their motivation for participating. A better understanding of how students are increasing this knowledge and the cause of it could lead to better retention.

Table 4.2

idesign assessment instruments results summary

\begin{tabular}{|l|c|c|c|c|c|c|c|c|}
\hline \multicolumn{3}{|c|}{ Readiness } & \multicolumn{3}{c|}{ IDI } & Motivation & \multicolumn{3}{|c|}{ SESL } \\
\hline & Pre & Pre & Post & Attrition & Pre & Pre & Post & Attrition \\
\hline $\begin{array}{l}\text { \# of } \\
\text { Students }\end{array}$ & 42 & 23 & 23 & 32 & 16 & 9 & 9 & 22 \\
\hline $\begin{array}{l}\text { \% } \\
\text { Female }\end{array}$ & $59 \%$ & $61 \%$ & $61 \%$ & $66 \%$ & $56 \%$ & $44 \%$ & $44 \%$ & $64 \%$ \\
\hline Score & & & & & & & \\
Max & 52 & 116.3 & 109.5 & 116.2 & 100 & 89.7 & 90 & 91.0 \\
\hline Min & 13 & 71.4 & 60.3 & 63.8 & 63.3 & 56 & 60.6 & 55.7 \\
\hline Average & 34.2 & 86.0 & 85.2 & 87.5 & 84.7 & 71.8 & 75.1 & 78.1 \\
\hline StDev. & 10.0 & 9.5 & 12.8 & 12.7 & 11.0 & 10.5 & 7.6 & 9.5 \\
\hline Range & $0-60$ & & $55-145$ & $0-100$ & & $0-100$ \\
\hline
\end{tabular}


Table 4.3

idesign Readiness and Intercultural Development Inventory results summary by gender

\begin{tabular}{|l|c|c|c|c|c|c|c|c|c|}
\hline \multicolumn{1}{|c}{ Readiness } & \multicolumn{1}{c}{ IDI Pre } & IDI Post & IDI Attrition \\
\hline & Male & Female & Male & Female & Male & Female & Male & Female \\
\hline $\begin{array}{l}\text { \# of } \\
\text { Students }\end{array}$ & 17 & 25 & 9 & 14 & 9 & 14 & 11 & 21 \\
\hline Score & & & & & & & \\
\hline Max & 46 & 52 & 90.7 & 116.3 & 109.5 & 108.5 & 105.4 & 116.2 \\
\hline Min & 13 & 16 & 74.3 & 71.4 & 67.8 & 60.3 & 71.9 & 63.8 \\
\hline Average & 31.7 & 36.0 & 82.1 & 88.4 & 83.2 & 86.6 & 85.5 & 88.5 \\
\hline StDev. & 9.3 & 10.4 & 6.3 & 10.6 & 15.7 & 10.9 & 10.2 & 14.0 \\
\hline Range & \multicolumn{7}{|c|}{$0-60$} & & \multicolumn{7}{|c|}{$55-145$} & & \\
\hline
\end{tabular}

Table 4.4

idesign Motivation and SESL results summary by gender

\begin{tabular}{|l|c|c|c|c|c|c|c|c|c|}
\hline \multicolumn{9}{|c}{ Motivation } & \multicolumn{2}{c|}{ SESL Pre } & SESL Post & \multicolumn{2}{c|}{ SESL Attrition } \\
\hline & Male & Female & Male & Female & Male & Female & Male & Female \\
\hline $\begin{array}{l}\text { \# of } \\
\text { Students }\end{array}$ & 17 & 25 & 5 & 4 & 5 & 4 & 8 & 14 \\
\hline Score & & & & & & & \\
\hline Max & 100 & 100 & 83.4 & 89.7 & 76.9 & 90 & 88.9 & 90.9 \\
\hline Min & 77.8 & 75.6 & 67.1 & 56 & 73.1 & 60.6 & 63.4 & 55.7 \\
\hline Average & 88.5 & 84.9 & 74.2 & 68.9 & 75.0 & 75.2 & 75.5 & 79.6 \\
\hline StDev. & 8.6 & 7.5 & 6.7 & 14.7 & 1.75 & 12.2 & 9.4 & 9.51 \\
\hline Range & $0-100$ & & \multicolumn{7}{|c|}{$0-100$} \\
\hline
\end{tabular}

\subsection{Qualitative Results}

Of the methods outlined in Chapter 2, word cloud and motivational narrative analysis were utilized for the idesign program. The interview protocol is in the early testing stages and has not been implemented on a full scale at this time. Future analyses will include Word clouds, Motivational narrative analysis, along with interviews utilizing the protocol outlined previously.

\subsubsection{Word Cloud}

In order to initially examine the motivational narratives and find some general tendencies, word clouds were utilized. These tag clouds can be beneficial in preliminary evaluation when it's not quite certain what should be found (Sinclair 2008). The word cloud allows the viewer to visualize all of the text at once without having to look through each of the individual text files. Figure 4.2 helps to demonstrate all of the idesign motivational narrative code uses at once. For the idesign participants the words Helping and Others come across as being the largest, suggesting "Helping Others" is the most commonly occurring code for these motivational narratives. These preliminary 
results are further explored in the frequency reports summarized in the remainder of this chapter.

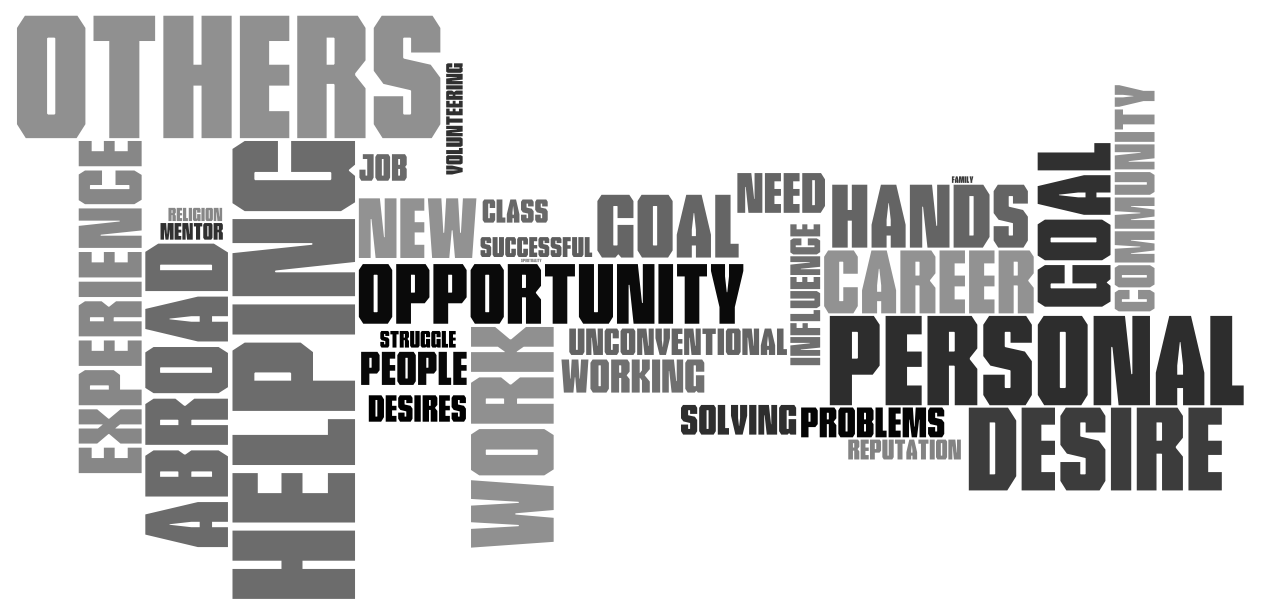

Figure 4.2: Word cloud of motivation codes for idesign motivational narratives $(n=36)$

\subsubsection{Motivations}

In addition to the motivational analysis utilized in the ISES survey within the quantitative analysis, it is also used for the purposes of understanding the results of the motivational narratives. As outlined in Chapter 2, motivational codes were created using the in vivo coding process. This section outlines the results of analysis of the frequency reports created utilizing the codes attempting to address the first three hypotheses addressing the impact of service learning experiences, gender, and education level.

\subsubsection{Gender Influences}

Despite decades of effort, the engineering field is still dominated by white men. Intriguingly, Learning Through Service (LTS) programs, especially international ones, are disproportionately comprised of women, typically 50\% (Bielefeldt et al. 2010; Paterson and Fuchs 2008; Parkinson 2007; Busch-Vishniac and Jarosz 2004). A better understanding of what is attracting (and retaining) females to these programs could create pathways enabling universities to effectively create student bodies more representative of society. The evaluation of the gender-filtered code frequency reports effectively illuminate what attracts males and females to the international senior capstone design program at Michigan Tech; these are likely a representation of what could be seen at other universities in other programs similar to this, but a greater study pool will elevate the confidence of generalized findings. Tables 4.3 reveals demographic patterns by gender: a key finding is the female participation rate $(64 \%)$; there is a strong interest in international programs from women, even though they tend to have less international experience than men in these programs (Figure 4.2). This interest could be attributed to some of their motivating factors; females may see an opportunity to help others in a way they haven't been able to in other aspects of engineering, it could be career related, or they might find this to be a perfect opportunity to work with a community in need. Further analyses of the motivations suggest that the top two reasons are exactly the same, although the break-down for the third, fourth, and fifth reasons are 
different (Table 4.5). Males and females alike agree that helping others, their desire to work abroad, the opening for new opportunities, the availability of hands on experiences, and their own personal goal are the main five reasons (females have two additional reasons) that motivate them to participate in these programs. Some examples of students expressing their engineering design motivation associated with helping others include:

As an engineer, I have a unique capacity (and corresponding responsibility) to put science and technology to work for those in need.

I hope to use my technical skills and the social and cultural awareness I gained from Semester at Sea to help my host community develop water and sanitation infrastructure that meet their needs and desires.

Additionally idesign exemplifies their engineering design motivations associated with their desire to work abroad:

I feel that international senior design will help fill the gaps left in my experience from not being able to get work experience or study abroad. I am very excited about the opportunities that this program has to offer.

These example quotes along with the findings within Table 4.6 help to support the claim that female students are more motivated to participate in engineering design programs when given the opportunity to apply what they are learning to help others. However, males are also motivated by these same factors and there is no way to determine at this point if females are more motivated than males to do engineering design tasks. The reasoning behind why males and females comment that these are motivating factors may differ, however, the use of this knowledge can still be applied within the classroom or recruitment efforts to better retain and attract students to engineering.
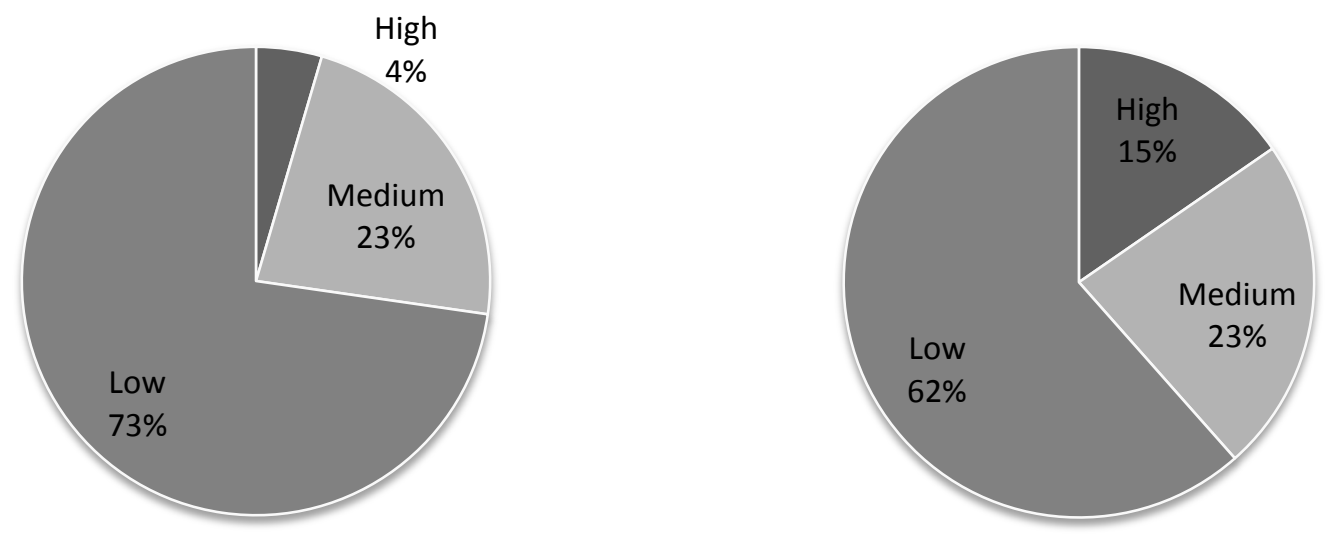

Figure 4.3: International experience for (left to right): all idesign women $(n=23)$ and all men $(n=13)$. Low is 0 to 10 weeks, medium is 10 to 30 weeks, and high is greater than 30 weeks of living and traveling internationally 
Table 4.5

idesign demographics

\begin{tabular}{|l|c|}
\hline Metric & idesign Cohort \\
\hline Number of Students & 55 \\
\hline Gender (\% female) & $64 \%$ \\
\hline Prior experience abroad (weeks) & 11.2 \\
\hline Proficiency in non-native language(s) & 2.6 \\
\hline
\end{tabular}

Table 4.6

Top five motivations expressed by idesign men and women; rank (frequency), $n=214$ code tags within 36 student essays

\begin{tabular}{|l|c|c|}
\multicolumn{1}{|c}{ Motivation } & \multicolumn{1}{c}{$\begin{array}{c}\text { Male Rank } \\
\text { (frequency) }\end{array}$} & \multicolumn{1}{c|}{$\begin{array}{c}\text { Female Rank } \\
\text { (frequency) }\end{array}$} \\
\hline Helping others & $1(19 \%)$ & $1(16 \%)$ \\
\hline Desire to work abroad & $2(14 \%)$ & $2(11 \%)$ \\
\hline New opportunities & $3(11 \%)$ & $5(7 \%)$ \\
\hline Hands on experience & $4(9 \%)$ & $4(9 \%)$ \\
\hline Personal goals & $5(8 \%)$ & $2(11 \%)$ \\
\hline Career goals & & $3(10 \%)$ \\
\hline Community need & & $5(7 \%)$ \\
\hline
\end{tabular}

\subsubsection{Academic Level Influences}

Statistics also reveal that fewer students are pursuing higher-level degrees and that the majority finishes their undergraduate program and go directly into the work force (NSB 2008; NSF 2011). Understanding what the motivations are of the two levels of students might help encourage students, who might not have otherwise considered it, to explore these experiences and continue their education at the graduate level. The motivations of each level of student were analyzed within this study and found that although the graduate program (further discussed in Chapter 5) has lower numbers, it attracts individuals with higher international experience (even normalized for age; data not shown). This, however, does not provide insight in the motivation of participants; it does provide a better understanding of the background of the students. Some of the same top three reasons as with males and females were found to be the case for undergraduates and graduates; they are motivated by helping others, their desire to work abroad, and their own personal goals, with the addition of solving problems as a top motivator for graduate students (Table 4.7). Some additional examples of students expressing their motivation to do engineering design include:

\section{I feel that iDesign would be a great way to help reach my goal of working} abroad in a third-world community.

By partaking in this project, I would not only have the joy of knowing that the work I was putting into my education was helping the lives of others, but I would 
also be gaining significant career experience in a unique field of construction where experience is often very difficult to acquire.

These quotes help to demonstrate students represented in Table 4.7, while providing some additional insight on their perspectives. Since the top reasons were insensitive to class ranking, the second tier reasons were further examined. Undergraduates were more motivated by pragmatic reasons, like their career aspirations, as the previous quotes help to exemplify. It is clear that the reasoning between these two levels require a very different approach in attracting more individuals to these programs. Many undergraduates want to see the professional development advantages of participation. Although these observations provide some initial insight on whether highly education students are more motivated to do engineering design tasks, they cannot provide conclusive statistical evidence to support this claim. Chapter 5 further explains graduate student tendencies.
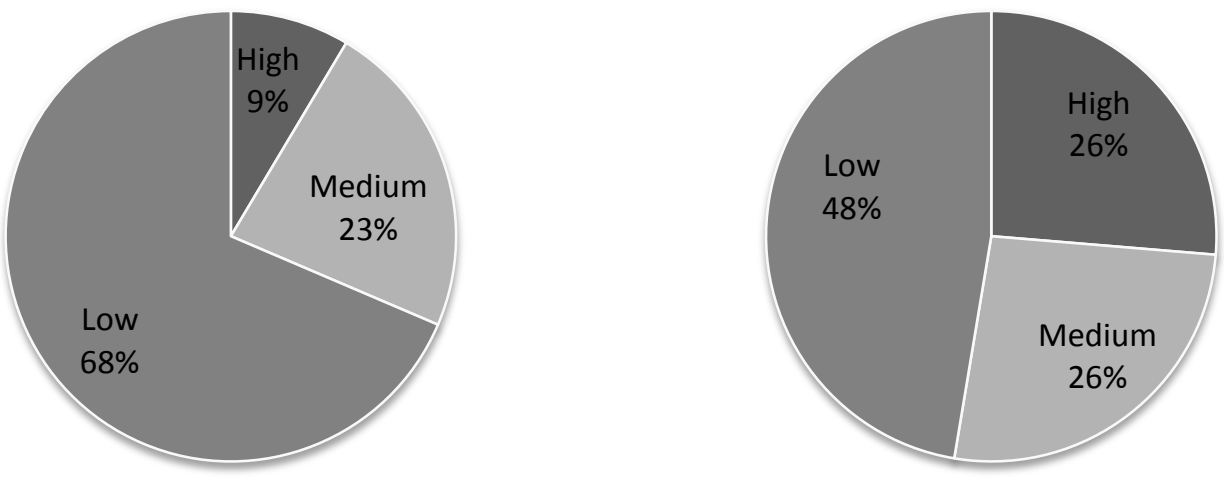

Figure 4.4: International experience for (left to right): all undergraduates $(n=36)$ and all graduate students $(\mathrm{n}=19)$. Low is 0 to 10 weeks, medium is 10 to 30 weeks, and high is greater than 30 weeks of living and traveling internationally

Table 4.7

Top five motivations expressed by undergraduate and graduate students; rank (frequency), $\mathbf{n = 3 3 2}$ tags within 55 student essays

\begin{tabular}{|l|c|c|}
\hline Motivation & $\begin{array}{c}\text { Undergraduate } \\
\text { rank (frequency) }\end{array}$ & $\begin{array}{c}\text { Graduate } \\
\text { rank (frequency) }\end{array}$ \\
\hline Helping others & $1(17 \%)$ & $1(15 \%)$ \\
\hline Desire to work abroad & $2(12 \%)$ & $4(8 \%)$ \\
\hline Personal goals & $3(10 \%)$ & $2(12 \%)$ \\
\hline Solving problems & & $3(9 \%)$ \\
\hline Career goal & $4(9 \%)$ & $5(7 \%)$ \\
\hline Hands on experience & $4(9 \%)$ & \\
\hline New opportunity & $5(8 \%)$ & \\
\hline Successful reputation & & $5(7 \%)$ \\
\hline
\end{tabular}




\subsubsection{Intercultural Experience Influences}

Once students become involved with international programs, it is often difficult to go back to their daily lives without craving more. Students who have previously had international opportunities were also analyzed to determine what their motivations were for becoming involved with each of these two programs. The hope was that gathering information about whether an additional international experience was enough to attract the student or if they had alternative motivations. Students were broken up into three categories for this category of analysis: low (0 to 10 weeks), medium (10 to 30 weeks) and high (above 30 weeks) international experience. Three categories were selected over two (some or none) to differentiate those students who have had experiences abroad in a higher capacity (like a study abroad), from those who have had family vacations or very little (to no) experience abroad. The frequency results show these programs attract a fair amount from each level (Figures 4.2 and 4.3). In fact, the undergraduate program is the first international experience for $27 \%$ of the cohort. Some students are interested in sampling such experiences for the first time; many others are returning for more. If the experiences are positive, the biggest hurdle is crafting first experiences, and then a virtuous cycle of involvement can be catalyzed. Programs like idesign need to be developed extensively to ensure students who have never had an international experience have a positive one. The results of participants' motivations hope to determine if in fact individuals who have positive previous international experiences are more motivated to pursue others where they can additionally utilize their engineering design skills

Similarly to previous sections, top reasons for all international experience levels are helping others, desire to work abroad, and personal goals. Yet further examination reveals a few interesting differences. Desire to help others decreases with experience, this may be rooted an appreciation of the realities of development work (partnership oriented vs. "helping"). As students have more experiences in developing work, they can come to find it requires a lot more effort than they initially anticipated, yet they still find enjoyment in travel and new experiences. The most experienced students ranked a desire to work abroad most highly. From these preliminary observations it seems that new (less experienced) students may connect more with an "engineering philanthropy" goal, whereas experienced students are looking for "engineering development." Regardless of their mindset, encouraging students to become involved with these programs because of the opportunity to help others and gain valuable experience should be attractive.

Additional information pertaining to graduate student motivation is discussed further in Chapter 5. 
Table 4.8

Top three motivations expressed by idesign and PCMI students with low, medium, and high international experience; rank (frequency), $n=332$ code tags within 55 student essays

\begin{tabular}{|l|c|c|c|}
\hline \multicolumn{1}{|c}{ Motivation } & $\begin{array}{c}\text { Low rank } \\
\text { (frequency) }\end{array}$ & $\begin{array}{c}\text { Medium rank } \\
\text { (frequency) }\end{array}$ & $\begin{array}{c}\text { High rank } \\
\text { (frequency) }\end{array}$ \\
\hline Helping others & $1(17 \%)$ & $1(16 \%)$ & $1(15 \%)$ \\
\hline Personal goals & $2(12 \%)$ & $3(9 \%)$ & $3(11 \%)$ \\
\hline Desire to work abroad & $3(11 \%)$ & & $2(13 \%)$ \\
\hline Career goals & & $2(13 \%)$ & $2(13 \%)$ \\
\hline
\end{tabular}

\subsubsection{Interview Protocol}

The interview process for the idesign program is only in the initial stages. Some interviews have been conducted using similar questions to the PCMI program (Chapter 5); however, results are unavailable at this time. Future work will report the results of the interviews and discuss next steps of this method.

\subsection{Conclusions}

The chapter started out with the goal of determining what motivates the idesign students to do engineering design work? The various instruments utilized within the qualitative and quantitative methodologies helped to evaluate hypotheses developed in response to this question and came to some initial conclusions. These specific hypothesis and their results are further summarized below.

The claim that students who self-report higher levels of readiness to participate in their respective engineering program are more motivated with respect to engineering design tasks was evaluated using the pre scores of idesign participants. Unfortunately, due to the observational nature of the analysis for the readiness data at this time, a statistical confirmation could not be reached. These preliminary scores provide some insights, suggesting that students are ready to participate in engineering design task, which helps to support the initial claim. As this study continues to collect participate pre and post Readiness scores, further analysis can be done to determine if those with higher scores are in fact more motivated to do engineering design work.

Next the claim that students with higher levels of intercultural competency are expected to have a better understanding of other cultures, leading them to be better equipped and more motivated to perform engineering design tasks in other societies was evaluated using the IDI scores. The preliminary findings for the idesign participants suggest that their average is actually decreasing from pre to post international, thus the initial claim cannot be supported by the data at this time. Future efforts should be put towards preparing students for fieldwork to increase their intercultural competency. Additionally, as the study continues to collect participant information, further evaluations will be done to determine if this is a consistent trend over time.

Students' understanding of sustainable engineering was also evaluated to determine if in fact a better understanding results in higher motivations to utilize these skills with regard 
to engineering design. The results suggest that idesign students are increasing their knowledge of sustainable engineering, which helps to support this claim. Additionally, however, these results are based off of observational analysis and cannot statistically confirm the hypothesis. Participants of future cohorts coming into the idesign program will be utilized to analyze the data to provide some statistical evidence to support the hypothesis.

Next gender was examined to determine if female students are more motivated to participate in engineering design programs when given the opportunity to apply what they are learning to help others. The frequency reports resulting from the motivational narratives provided a basis to claim that idesign female students are motivated by applying their knowledge and skills, as well as by helping others. However, male students alike are motivated by these factors. Additionally, higher scores in the selfperceived level of Readiness to do engineering design work, the Intercultural Development Inventory results, along with aspects associated with sustainable engineering (post and attrition categories) help to support females being more motivated to do engineering design work. Statistical analysis will be utilized in future efforts, as more participants are added, to determine if the aforementioned hypothesis can be proven.

It is suggested that highly educated students are motivated to do engineering design tasks at higher levels when utilizing their skills. This was evaluated using ethnographic analyses of the motivational narratives. Although the results presented similar findings to the gender analysis, it did not provide enough evidence to claim education impacts the level of motivation to do engineering design tasks. Additionally, future participant cohorts will be included to conduct statistical analysis to help prove education impacts motivation.

Lastly, students' international experience was evaluated to determine if individuals who have had have positive previous international experiences are more motivated to pursue others where they can additionally utilize their engineering design skills. The motivational narratives' frequency reports were also utilized in this observational analysis. It is clear from these participants that more international experience results in a wider range of motivating factors; however, it is inconclusive in determining if more previous international experience results in higher levels of motivation to do engineering design tasks. As previously mentioned, as more information is collected, this analysis will be revisited to determine if this hypothesis can be proven with statistical confidence.

These various attributes of student participants were evaluated to help determine what is motivating students to do engineering design work. As more is learned about the motivations, the program can be altered to enhance its effectiveness and attractiveness for future participants.

\subsection{Next Steps}

This study is longitudinal in nature; more cohorts will continue to go through the program and information will continue to be collected and analyzed to better understand 
idesign participants. As the collection of data from participants increases, more evidence will be available to make claims about student motivations, as well as about what students are getting out of their involvement in the program. This understanding can have a considerable impact on the student experience, leading to increased interest and better retention for the idesign program. 


\section{Peace Corps Master's International}

This chapter focuses on a crosscutting research opportunity in comparison to the more narrowed focus on one academic program as discussed in the previous chapter. Participants in this program have the capability of utilizing their skills in a variety of ways in addition to being able to incorporating some of their own programmatic decisions (i.e. course selections and location preference for service). Some of the initial findings of the assessment instruments are revealed, in spite of the limited numbers, within this chapter and provide a basis for which similar programs can start. Focuses of these instruments will be on those that pertain to motivation.

\subsection{Background}

The Peace Corps Master's International (PCMI) program in civil and environmental engineering is a graduate level program at Michigan Tech University which bridges a typical Master's level degree with service in the Peace Corps. Students in this program spend approximately one year in coursework preparing for their 27-month fieldwork component (completed during service). Upon completion of their fieldwork students spend an additional semester on campus reflecting on their work and presenting their findings in the form of a thesis defense. Students are asked to complete online surveys and write a motivational narrative upon entering this program. Additional surveys are requested again upon completion of the coursework portion of the program. The narratives and questions within the surveys collect motivational data of which this paper will focus on those initial findings focusing on students who started the program Fall 2010 and Spring or Fall of 2011.

\subsection{Program Description}

Very few graduate programs allow their students to complete a portion of their graduate work while serving in the Peace Corps. This ongoing study hopes to utilize the information being collected to better understand the needs and motivations of student participants as part of a larger study of the stakeholders. This phase will be used in future on-going studies, which will eventually be catered towards not only the student participants, but also the faculty, community, partnering organizations, the institutions the students are studying with, Peace Corps, as well as alum. This chapter aims initially to address the following aforementioned hypotheses: 1) Female students are more motivated to participate in engineering design programs when given the opportunity to apply what they are learning to help others, 3) Student who self-report higher levels of readiness to participate in their respective engineering program are more motivated with respect to engineering design tasks, 4) Students with higher levels of intercultural competency are expected to have a better understanding of other cultures, leading them to be better equipped and more motivated to perform engineering design tasks in other societies, 5) A better understanding of sustainable engineering should result in higher levels of student motivation to utilized these skills with regard to engineering design, 6) Highly educated students are motivated to do engineering design tasks at higher levels when utilizing their skills, and 7) Individuals who have positive previous international experiences are more motivated to pursue others where they can additionally utilize their engineering design skills. This data will be used to determine what is motivating 
participants to pursue engineering design work and will eventually be used to address stakeholder needs within the overall program.

\subsection{Program Assessment}

The same methods for the idesign program were used for the PCMI students (Table 5.1), including the interview protocol, which is in the process of being transitioned into the formalized assessment program. This will replace the piecemeal format we currently have where instruments have been added when a need arises. The hope of this approach is to continue to obtain the same information over time to be able to begin to understand the program and the participants. Students were asked to complete these assessments as they first started into the program and then again prior to departure for service. The collection of this information is on-going in an effort to build up a larger database of information to better understand the programmatic needs and student motivations for participating, while additionally providing a means to personalize these programs. Figure 5.1 depicts the spread of information pertaining to a subset of this particular cohort being examined.

Table 5.1

PCMI assessment instruments

\begin{tabular}{|l|c|}
\hline \multicolumn{1}{|c|}{ Instruments } & PCMI \\
\hline Readiness Assessment & $\mathrm{X}$ \\
\hline Intercultural Development Inventory & $\mathrm{X}$ \\
\hline ISES (Motivation) & $\mathrm{X}$ \\
\hline SESL & $\mathrm{X}$ \\
\hline Motivational Narratives & $\mathrm{X}$ \\
\hline Interview Protocol & $\mathrm{X}$ \\
\hline Word Cloud & $\mathrm{X}$ \\
\hline
\end{tabular}




\subsection{Design of Study}

This program creates a unique opportunity to join traditional Master's research with service in the U.S. Peace Corps, providing a substantial 27-month international experience. These students spend a much larger amount of time living within a community giving the advantage for data collection and communication with their community. At this point, there are limitations with available data due to the length of time students spend abroad; many have not returned from service to finish the follow up component (i.e. post) of the assessment yet. Thus the preliminary analysis is limited to those who have completed the assessment instruments. As the program continues to grow and attract new students, further changes and program personalization opportunities will be available for participating students (i.e. suggesting specific coursework to better suit student needs and interests). The focus for this chapter will be on those that have completed the assessments, as well as the motivational components of those assessments.

\subsection{Quantitative Results}

Due to the nature of collecting survey data, these surveys vary in number of participants. Table 5.1 demonstrates some demographics for the highest recorded population size for the survey data, which is further examined in this chapter. The instruments outline in Chapter 2 aim to help explain how service learning involvement, gender influence, and education level influence student motivation related to engineering design work. The evaluation of these instruments is presented below.

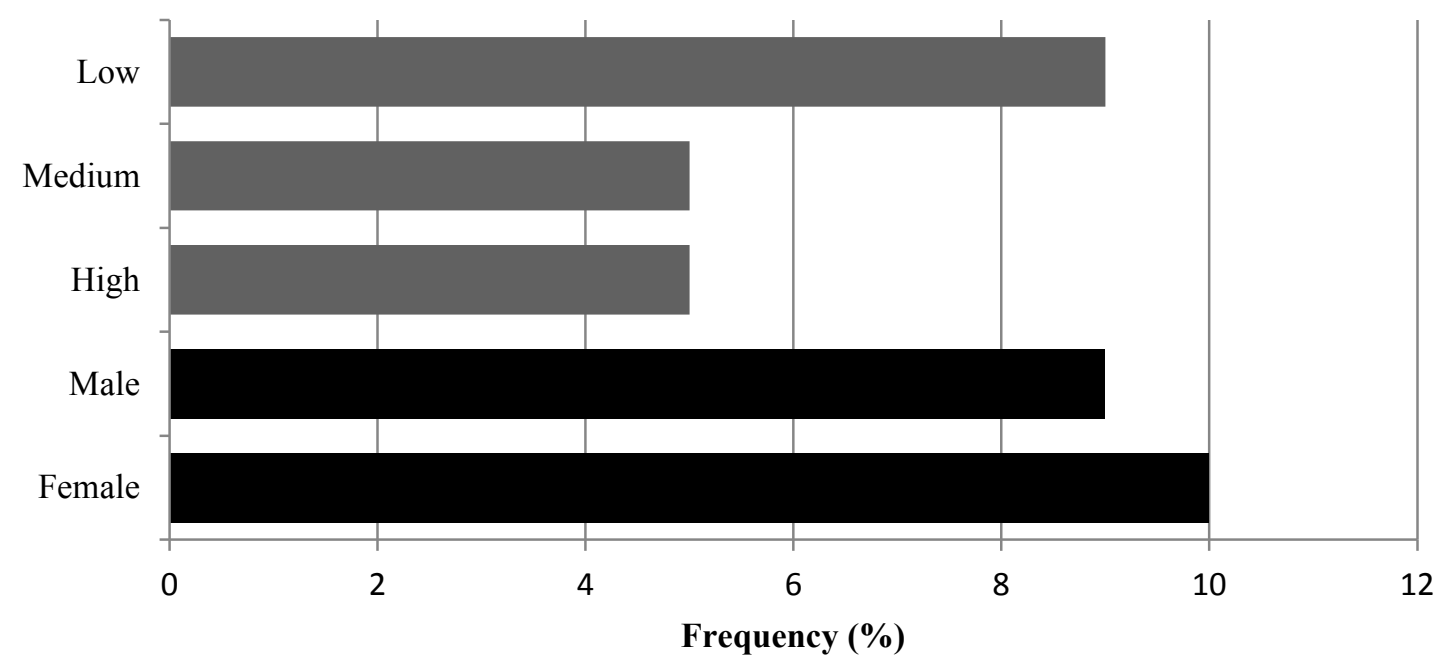

Figure 5.1: PCMI Student participant demographics in two categories: gender, and prior international experience $(n=36)$ 


\subsubsection{Readiness}

As discussed in Chapter 4, the readiness assessment was implemented to understand how well prepared students are to participate in the PCMI program, which is additionally used to understand students' self-assessment of their Readiness to do engineering design work. The tabulated results determined the self-assessment of Readiness for graduate students was lower than undergraduate students. This could imply that graduate students are more realistic about their upcoming experience. Additionally, undergraduate students were assessing themselves for a two-week out-ofcountry program, whereas the graduate students were assessing for seven semesters (27 months) abroad, which could provide further insight as to why graduate students scored lower than undergraduates. Although these programs are both very unique in nature, the comparison is being made to demonstrate how Readiness differs from program to program and understanding this can help to address student engineering design motivation overall. The long-term goal of gaining a better understanding of their motivation to participate is to be able to link this to their motivation to do engineering design tasks, which is a unique aspect of the PCMI program as compared to a general Peace Corps volunteer. This instrument is in the process of being added to the mid and/or post component of both programs; however, at this time data is only available for the mid component for the PCMI program. Despite the limitations associated with the data at this point, some increase in readiness is being seen thus far (Table 5.2).

Table 5.2 also demonstrates what students are leaving the study within the attrition column. This helps to better understand what kinds of students are being lost in the post Readiness scores. These students, however, are only present in the attrition column. Students in pre and post are the same individuals to give a better demonstration of changes over time. The initial results suggest that the maximum and averages are seeing some increases over time, which helps to support the claim that students' self-reporting higher levels of Readiness are more motivated to do engineering design tasks. Additionally, Table 5.4 demonstrates Readiness scores broken down by gender. This helps to support the claim that females are more motivated to do engineering design work as a result of their scores being higher than males. These claims, however, are not supported by statistical evidence, yet they lend some initial insights in the PCMI participants. Future efforts will incorporate additional cohorts in a statistical evaluation of the Readiness scores to help confirm the aforementioned hypothesis.

\subsubsection{Intercultural Competency}

Utilizing the same method outline in Chapters 2 and 4, the IDI results for graduate students are presented herein. For the graduate students, the mid scores represent their score at the time they are completing coursework prior to leaving for fieldwork. Postproject scores for graduate students will be available following their Peace Corps service (more than two years from now). The claim that students with higher levels of intercultural competency are expected to have a better understanding of other cultures, leading them to be better equipped and more motivated to perform engineering design tasks in other societies is evaluated using the IDI results. Table 5.2 demonstrates the results of the graduate IDI scores ( $p r e n=48$, mid $n=33$ ). The graduate students averages 
noticeably increase over the course of their time on campus, suggesting that they are becoming more culturally aware and are more prepared to work with people of different worldviews, which helps to support the claim. This change is seen over the time period they are on campus, which could be attributed to the courses they are taking as well as their own personal research pertaining to their upcoming Peace Corps assignments. Additionally, the gender breakdown is provided in Table 5.5, which helps to demonstrate higher scores for female participants in post and attrition categories. This begins to help support the claim that females are more motivated than males to do engineering design work. However, as the results of this evaluation are purely observational in nature, future analyses will be conducted as more participants are added to the study. The results of these analyses aim to confirm that intercultural competency impacts student motivation to do engineering design work.

\subsubsection{ISES (Motivation)}

Similar to the idesign program analysis, the PCMI program utilized the motivational scores to better understand student participants. The PCMI group has the advantage of having both a pre and a mid motivation score, giving a more in depth look at the students and the program, while the idesign group lacks post scores. Table 5.2 demonstrates the motivational results of the ISES survey for the PCMI program. Some preliminary analysis suggests motivational scores are increasing as a result of the oncampus portion of the PCMI experience. Additionally, Table 5.6 depicts the gender breakdown. This, however, does not support the claim that females are more motivated to do engineering design work as a result of higher scores. These scores will be utilized in future analyses to help determine what is impacting student motivation to do engineering design tasks and to determine if there are changes over time.

\subsubsection{SESL}

Evaluating the students' self-efficacy, beliefs, and interests related to Sustainable Engineering in Service Learning students resulted in Table 5.2, demonstrating the PCMI students change in SESL understanding over time. At this point, it is difficult to say why the maximums and minimums are staying relatively the same; this could be suggestive of students coming in with a higher level of appreciation for sustainable engineering than anticipated. However, future work and increasing the study pool will aim to address this question and determine if changes need to be made to improve students' sustainable engineering self-efficacy, beliefs, and interests. Tables 4.2 and 5.2 provide enough information to make some preliminary comparisons for both programs and demonstrates how the undergraduate students self-report a higher level of sustainable engineering selfefficacy, beliefs, and interests. Additionally, Table 5.2 shows the change in SESL, specifically that there is a negative change for PCMI students. These findings could be rooted in undergraduate students coming across over-confident about their understanding, whereas graduate students realize how much more there is to learn after leaving a structured undergraduate program and completing two semesters of graduate work. The results of this preliminary observational analysis was intended to determine if a better understanding of sustainable engineering results in higher levels of student motivation with regard to engineering design. The results, at this point, depict a decrease in SESL scores, which cannot support the aforementioned claim. A gender evaluation of 
the SESL scores is depicted in Table 5.7. This helps to support the claim that females are more motivated to do engineering design work, as their scores are relatively higher than males (with the attrition category being the exception). Future studies will additionally examine this as the participant pools continue to grow and more information is made available. At this point, this survey is used as an additional tool to evaluate the students' motivation with respect to engineering; however, additional information can also be obtained from this survey.

Table 5.2

PCMI Readiness and IDI assessment instruments results summary

\begin{tabular}{|l|c|c|c|c|c|c|}
\hline \multicolumn{7}{|c|}{ Readiness } \\
\hline & Pre & Mid & Attrition & Pre & Mid & Attrition \\
\hline $\begin{array}{l}\text { \# of } \\
\text { Students }\end{array}$ & 9 & 9 & 12 & 33 & 33 & 15 \\
\hline $\begin{array}{l}\text { \% } \\
\text { Female }\end{array}$ & $56 \%$ & $56 \%$ & $50 \%$ & $55 \%$ & $55 \%$ & $20 \%$ \\
\hline Score & & & & & & \\
\hline Max & 48 & 59 & 43 & 128.7 & 129.2 & 123.9 \\
\hline Min & 15 & 11 & -4 & 68.8 & 72.4 & 71.8 \\
\hline Average & 29.2 & 29.7 & 27.4 & 93.3 & 98.0 & 96.5 \\
\hline StDev. & 10.7 & 14.8 & 12.8 & 15.1 & 13.7 & 14.5 \\
\hline Range & \multicolumn{7}{|c|}{$0-60$} & & $55-145$ \\
\hline
\end{tabular}

Table 5.3

PCMI Motivation and SESL assessment instruments results summary

\begin{tabular}{|l|c|c|c|c|c|c|}
\hline \multicolumn{7}{|c|}{ Motivation } \\
\hline & Pre & Mid & Attrition & Pre & Mid & Attrition \\
\hline $\begin{array}{l}\text { \# of } \\
\text { Students }\end{array}$ & 5 & 5 & 8 & 18 & 18 & 3 \\
\hline $\begin{array}{l}\text { \% } \\
\text { Female }\end{array}$ & $40 \%$ & $40 \%$ & $50 \%$ & $61 \%$ & $61 \%$ & $33 \%$ \\
Score & & & & & \\
\hline Max & 100 & 95.6 & 100 & 84.2 & 91.1 & 88.3 \\
\hline Min & 78.9 & 77.8 & 61.1 & 18.2 & 75.7 & 80 \\
\hline Average & 89.1 & 84.7 & 85.7 & 61.8 & 82.7 & 84.3 \\
\hline StDev. & 7.8 & 7.4 & 13.6 & 21.7 & 4.6 & 4.2 \\
\hline Range & \multicolumn{7}{|c|}{$0-100$} & \multicolumn{4}{|c|}{$0-100$} \\
\hline
\end{tabular}


Table 5.4

PCMI Readiness assessment instruments results summary by gender

\begin{tabular}{|c|c|c|c|c|c|c|}
\hline & \multicolumn{2}{|c|}{ Readiness Pre } & \multicolumn{2}{|c|}{$\begin{array}{c}\text { Readiness } \\
\text { Mid }\end{array}$} & \multicolumn{2}{|c|}{$\begin{array}{c}\text { Readiness } \\
\text { Attrition }\end{array}$} \\
\hline & Male & Female & Male & Female & Male & Female \\
\hline $\begin{array}{l}\text { \# of } \\
\text { Students }\end{array}$ & 4 & 5 & 4 & 5 & 6 & 6 \\
\hline \multicolumn{7}{|l|}{ Score } \\
\hline Max & 32 & 48 & 33 & 59 & 42 & 43 \\
\hline Min & 15 & 19 & 11 & 28 & -4 & 19 \\
\hline Average & 23.3 & 34 & 18.8 & 38.4 & 24.7 & 30.2 \\
\hline StDev. & 7.4 & 11.2 & 10.1 & 12.1 & 16.7 & 8.2 \\
\hline Range & \multicolumn{6}{|c|}{$0-60$} \\
\hline
\end{tabular}

Table 5.5

PCMI IDI assessment instruments results summary by gender

\begin{tabular}{|c|c|c|c|c|c|c|}
\hline & \multicolumn{2}{|c|}{ IDI Pre } & \multicolumn{2}{|c|}{ IDI Mid } & \multicolumn{2}{|c|}{ IDI Attrition } \\
\hline & Male & Female & Male & Female & Male & Female \\
\hline $\begin{array}{l}\text { \# of } \\
\text { Students }\end{array}$ & 15 & 18 & 15 & 18 & 12 & 3 \\
\hline Score & & & & & & \\
\hline Max & 128.7 & 122.2 & 129.2 & 121.9 & 110.7 & 123.9 \\
\hline Min & 72.3 & 68.8 & 72.4 & 79.6 & 71.8 & 95.3 \\
\hline Average & 95.5 & 91.5 & 96.2 & 99.6 & 92.9 & 111.1 \\
\hline StDev. & 16.7 & 13.8 & 15.5 & 12.2 & 12.6 & 14.5 \\
\hline Range & \multicolumn{6}{|c|}{$0-60$} \\
\hline
\end{tabular}

Table 5.6

PCMI Motivation assessment instruments results summary by gender

\begin{tabular}{|c|c|c|c|c|c|c|}
\hline & \multicolumn{2}{|c|}{ Motivation Pre } & \multicolumn{2}{|c|}{$\begin{array}{c}\text { Motivation } \\
\text { Mid }\end{array}$} & \multicolumn{2}{|c|}{$\begin{array}{c}\text { Motivation } \\
\text { Attrition }\end{array}$} \\
\hline & Male & Female & Male & Female & Male & Female \\
\hline $\begin{array}{l}\# \text { of } \\
\text { Students }\end{array}$ & 3 & 2 & 3 & 2 & 4 & 4 \\
\hline \multicolumn{7}{|l|}{ Score } \\
\hline Max & 92.2 & 86.7 & 100 & 95.6 & 95.6 & 100 \\
\hline Min & 86.7 & 77.8 & 78.9 & 77.8 & 68.9 & 61.1 \\
\hline Average & 88.9 & 83.3 & 89.4 & 86.7 & 85.8 & 85.6 \\
\hline StDev. & 2.9 & 4.8 & 14.9 & 12.5 & 11.7 & 17.1 \\
\hline Range & \multicolumn{6}{|c|}{$0-60$} \\
\hline
\end{tabular}


Table 5.7

PCMI SESL assessment instruments results summary by gender

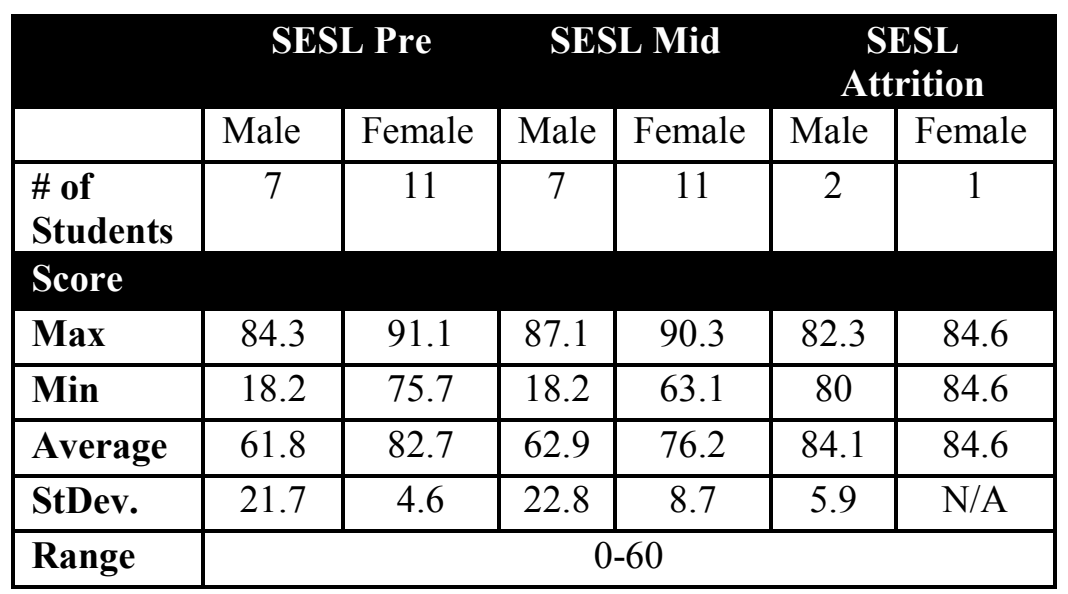

\subsection{Qualitative Results}

Similar to Chapter 4, Word cloud and Motivational narrative analysis was utilized to better understand the PCMI motivations. This section aims to evaluate and report some initial findings from the motivational narratives.

\subsubsection{Word Cloud}

In an effort to find some preliminary understanding to the motivational narratives provided by the PCMI students, a word cloud was created. This demonstrates the tagged codes within the narratives. The larger the word, the more often it occurred. Figure 5.2 depicts "Helping," "Others," and "Personal" as being the largest codes occurring in the motivational narratives. This helps to suggest that "Helping Others" and "Personal Goal" are the top two motivational factors for the PCMI students. This served as a starting point to analyze the narratives; however, further analyses of the frequency reports created from the flagged codes are discussed in the remainder of the chapter.

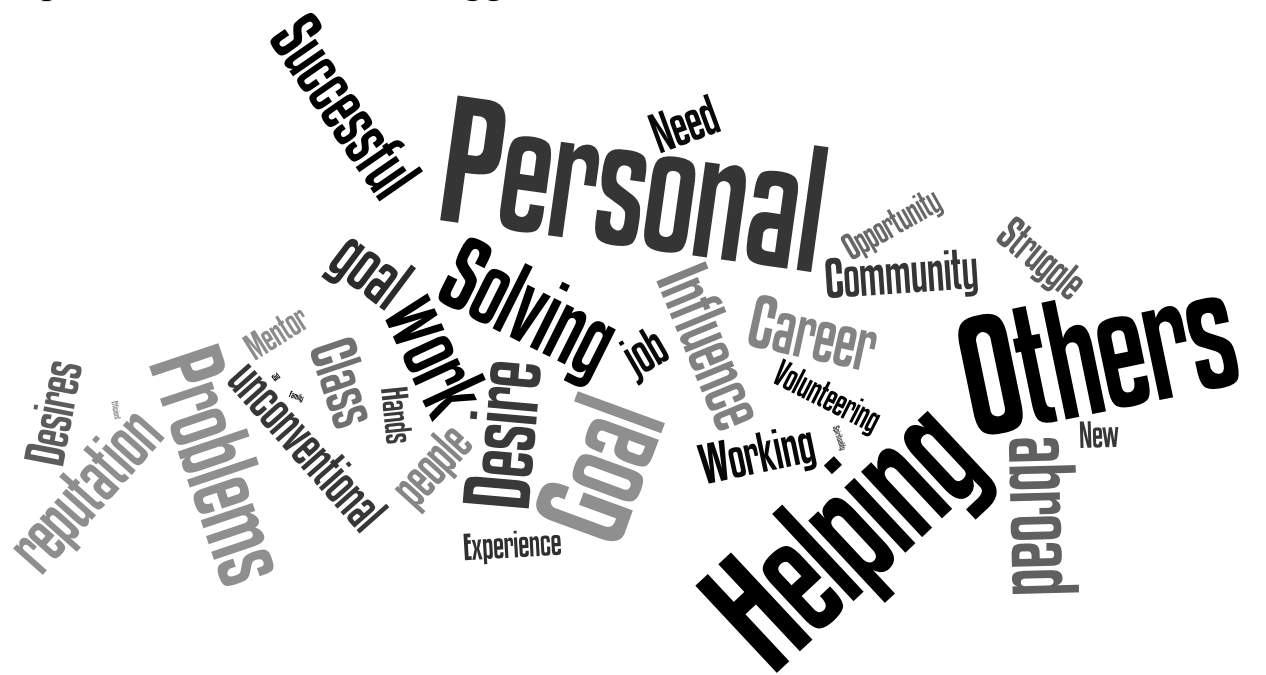

Figure 5.2: Word cloud of motivation codes for PCMI motivational narratives (n=19) 


\subsubsection{Motivations}

The motivational narratives are additionally examined for the PCMI students to better understand what is influencing their decision to participate in the program. This section evaluates gender, academic, and intercultural experience influence on student motivations in an effort to address the hypotheses outline in the objectives.

\subsubsection{Gender Influences}

In order to better understand student engineering design motivations, the flagged motivation codes were examined individually for males and females. Demographic information pertaining to the students being evaluated can be found in Table 5.3. One important characteristic of the data to note is the female participation rate $(54 \%)$; there is an obvious female presence in spite of their limited previous international experience (Figure 5.14). The goal in this analysis is to help support the claim that female students are more motivated to participate in engineering design programs when given the opportunity to apply what they are learning to help others in an effort to better understand what motivates students to do engineering design tasks. Examining PCMI student motivations, it is apparent they tend to fall into two broad categories, idealistic and pragmatic. The top reasons presented by the first group are: wanting to make a difference by helping others and fulfilling personal goals, as previously mentioned in the Word cloud. PCMI student examples describing their motivations include:

\section{I discovered that PCMI students from Michigan Tech finish many successful projects and have 100\% completion rate thanks largely to the technical and societal preparation they receive. This combined with the fact that completing the program also means completing a Master's Degree, a long held goal of mine, convinced me to apply.}

\section{The Peace Corps Master's International program in Civil and Environmental Engineering is the first of (hopefully) many opportunities to do this through the rest of my career and life.}

The second group typically fell into more pragmatic reasons; wanting to solve problems and others like career goals. A common statement was the desire to do engineering that matters to their community partners, but also to them as students. There was also a special pride in belonging to a tribe of fellow students similarly motivated to go above and beyond the required expectations for graduation. These results help to support the aforementioned hypothesis; however, this is based off of observational analyses associated with the frequency reports.

Additionally, a glance at the overall breakdown of the motivations of everyone involved in this study shows that there are three main motivations: helping others, desire to work abroad, and personal goal (Table 5.5); these vary slightly with topic of categorization (see Appendix 8 for further code descriptions). A further examination of the entire group suggests that career goals, solving problems, new opportunities, and hands on experience are also high motivators for students to become involved with international programs (Table 5.4). As discussed within the remainder of this chapter, this 
information can be used to encourage individual groups of students to increase the interest in these programs and continue to meet the needs of students, as well as determine what is motivating them to pursue engineering design work. Future efforts will be made to analyze these findings with larger pools of participants to gain statistical support.
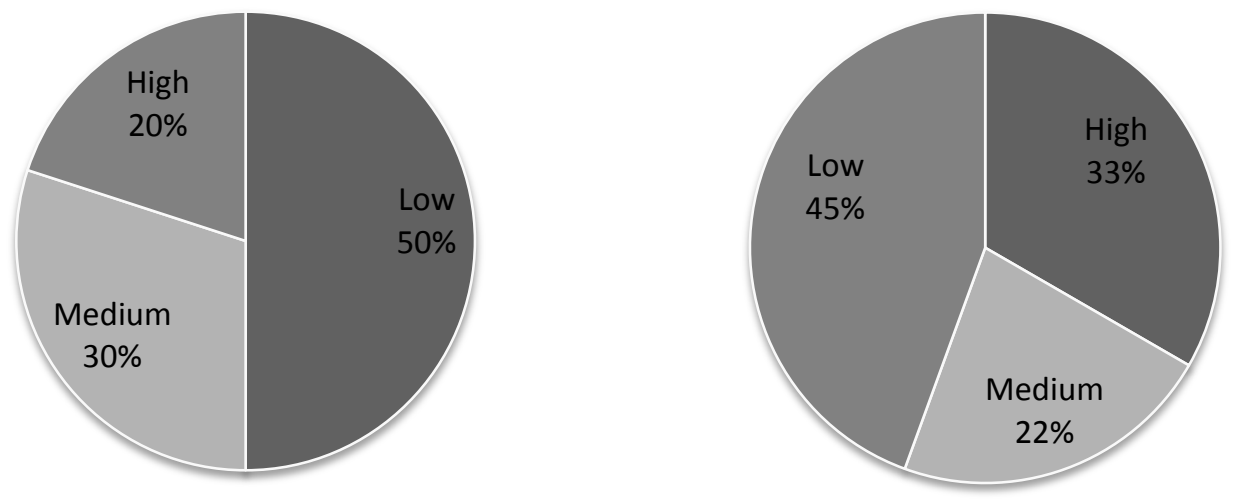

Figure 5.3: International experience for (left to right): all women $(n=10)$ and all men $(n=9)$. Low is 0 to 10 weeks, medium is 10 to 30 weeks, and high is greater than 30 weeks of living and traveling internationally

Table 5.8

PCMI demographics

\begin{tabular}{|l|c|}
\hline Metric & PCMI Cohort \\
\hline Number of Students & 48 \\
\hline Gender (\% female) & $54 \%$ \\
\hline Prior experience abroad (weeks) & 32.1 \\
\hline Proficiency in non-native language(s) & 2.8 \\
\hline
\end{tabular}

Table 5.9

Top five motivations expressed by men and women; rank (frequency), $n=118$ code tags within 19 student essays

\begin{tabular}{|l|c|c|}
\hline \multicolumn{1}{|c}{ Motivation } & $\begin{array}{c}\text { Male Rank } \\
\text { (frequency) }\end{array}$ & $\begin{array}{c}\text { Female Rank } \\
\text { (frequency) }\end{array}$ \\
\hline Helping others & $1(16 \%)$ & $1(15 \%)$ \\
\hline Personal goals & $2(12 \%)$ & $2(12 \%)$ \\
\hline Solving problems & $2(12 \%)$ & $4(7 \%)$ \\
\hline Desire to work abroad & $3(10 \%)$ & $4(7 \%)$ \\
\hline Career goals & $4(8 \%)$ & $5(6 \%)$ \\
\hline Successful reputation & $4(8 \%)$ & $5(6 \%)$ \\
\hline Working with people & $5(6 \%)$ & \\
\hline Desires unconventional job & $5(6 \%)$ & \\
\hline Class Influence & & $3(9 \%)$ \\
\hline New opportunity & & $5(6 \%)$ \\
\hline Community need & & $5(6 \%)$ \\
\hline
\end{tabular}


Table 5.10

Top motivation essay codes as response to reasons for participation in an international service program ( $=333$ code tags for 55 student essays)

\begin{tabular}{|l|c|}
\hline Motivation (code) & Frequency \\
\hline Helping others & $17 \%$ \\
\hline Personal goals & $11 \%$ \\
\hline Desire to work abroad & $11 \%$ \\
\hline Career goals & $8 \%$ \\
\hline Hands on experience & $7 \%$ \\
\hline New opportunities & $7 \%$ \\
\hline Solving problems & $6 \%$ \\
\hline
\end{tabular}

\subsubsection{Academic Level Influences}

As part of the graduate program within this study, students have the option to pursue a higher-level degree while gaining international experience. Adding to the evaluation discussed in Chapter 4, Table 4.4 helps to further demonstrate some of the graduate level tendencies. Intrinsic factors appear to be meaningful reasons for participating in the PCMI program (the desire to solve pressing problems, the satisfaction associated with being part of a well-regarded program, and the interest in having an unconventional career). It seems that these graduate level students require evidence (based on their philosophical, moral, and/or ethical views) that the program offers an opportunity to engineer a difference and they need more complex incentives to continue their education than undergraduates do since their objectives are less career and more personal. Although these observations are helpful in understanding the background of the PCMI students, which may eventually help to claim that higher levels of education result in higher motivation to do engineering design tasks, it does not provide insight in the motivation of participants or statistical evidence to say otherwise. However, this insight could be used as a basis for attracting future graduate students for the PCMI program. Targeting undergraduate students in the classroom and appealing to their interests to show them that there are other options than simply getting a degree and joining the traditional work force is essential for expanding programs like these.

\subsubsection{Intercultural Experience Influences}

As discussed in Chapter 4, the PCMI was evaluated with the idesign program to determine some general tendencies based off of international experience. Table 4.5 depicts the results of this analysis, suggesting there is one primary motivator: being able to help others by participating in the respective program. PCMI students, overall, tend to have more international experience than idesign participants (Table 5.2 and Figure 4.3), yet they have similar standards for being motivated to get involved. The goal of this information is to suggest that individuals who have positive previous international experiences are more motivated to pursue others where they can additionally utilize their engineering design skills. Unfortunately, this observational analysis of PCMI motivating factors is not sufficient to claim that the level of international experience has an impact on motivation for doing engineering design work. Utilizing this information, however, is 
another way to improve this program and work to attract individuals, not just the general public. As this study continues to collect PCMI data, statistical analysis will become more of an option to help support this claim.

\subsubsection{Interview Protocol}

The interview process for the PCMI program is in the initial stages, yet it has begun interviewing all of the out-going participants on a volunteer basis. Unfortunately, at this time results are not available for this part of the assessment instrumentation. The interview questions aim to gather information about their anticipations, concerns, along with various other data, which will help to determine what motivates participants to pursue engineering design work. The goal is to add onto the data currently being collected to gain a better understanding of the program's participants to continue to improve.

\subsection{Conclusions}

The evaluation of the PCMI students intended to explore various hypotheses to help determine what is motivating these particular students to pursue engineering design work. Although much of the results of this study come across inconclusive, the ethnographic nature of the results do provide some helpful insights on what is motivating students. The results of these explorations are further summarized below.

Readiness scores were evaluated to determine if they provided a basis for PCMI participants to be more motivated to do engineering design tasks. This was done an observational basis, which cannot provide statistical evidence, however it can provide some qualitative support and demonstrate the increase in scores. As further evidence is made available from larger participant pools and statistical analysis, this claim can be further proven.

Intercultural competency was also examined to determine if, in fact, students with higher levels are more motivated to perform engineering design tasks. The PCMI averages noticeably increased over their time on campus, which helps to suggest they are becoming more culturally aware, and therefore better prepared to work with people of different worldviews. These findings help to support the claim that they result in individuals who are more motivated to perform engineering design tasks. Statistical analysis of the data as the participant pool increases will help to reaffirm this claim.

Additionally, sustainable engineering knowledge scores were evaluated to determine if higher levels provoked higher levels of motivation in engineering design tasks. SESL scores were found to be decreasing at this point for the PCMI students, which cannot support the aforementioned claim. Students may be coming in with a higher level of knowledge, suggesting courses need to be adjusted to meet the needs of the incoming participants. Additionally, these findings may be a result of PCMI students realizing how much more they have to learn as a result of the courses they took while on campus, which lead to a lower score. Future analyses will continue to examine this to help determine what is motiving students to do engineering design work. 
The motivational narratives were utilized to determine if gender, level of education, or amount of previous international experience had an impact on motivation to do engineering design tasks. The thought behind this suggests that higher levels of education and amount of international experience should result in higher motivation levels. Additionally, it is argued that females are more motivated to do engineering design tasks given that they are able to utilize their learned knowledge to help others. Although the observational data provides some essential background information in understanding the PCMI participants, it cannot, however, help to claim that a higher level of education or more international experience impact the level of motivation. With respect to the observational evaluation of gender, there was some support found to help suggest that females are motivated by the opportunity to help others by utilizing their skills. Unfortunately, males also are motivated by these factors so the claim that females are more motivated cannot be made. Additionally, scores from the Readiness, IDI (post and attrition categories), and SESL instruments help to support females being more motivated to do engineering design work based off of their higher scores. Future evaluation of these independent variables using statistical methods concurrent with the qualitative methods utilized in this study can help to support these claims.

\subsection{Next Steps}

Moving forward, more data will be collected from future participants, which can be added to the data pool for continuing understanding of what students are getting out of their involvement as well as what is motivating them throughout the process to do engineering design work. Additionally, beta testing is being done to determine how data can be collected pertaining to how the experience affects students during their fieldwork portion. Future participant groups will be asked to participate in this "middle assessment" program to help gain perspective on the entire programmatic experience. In addition to the motivational analysis work being done on these individual programs, this realm is being explored on a curriculum level for engineering education as seen in Chapter 3. 


\section{Conclusions and Future Work}

Above all else the goal of this thesis was to provide an insight on why students are motivated to participate in engineering design tasks. The study additionally afforded some details on three engineering programs to provide a basis for other institutions to begin analyzing their own, similar programs. The methodology utilized to address engineering design motivations was divided into two categories: qualitative and quantitative. The results of these methods, along with how the results are tied into previous literature are further described below.

\subsection{Quantitative Methods Conclusions}

The quantitative methods outlined in Chapter 2 were utilized in evaluating all three of the community engagement programs discussed within the body of this thesis. Although the utilization of these methods varied from program to program, the general findings below use the aforementioned instruments in an effort to address some of the previously outlined hypotheses.

\subsubsection{ISES Quantitative Conclusions}

The on-going longitudinal study on engineering students' study utilized the online surveys constructed using several validated instruments (Chapter2). Engineering Design Motivational scores were used in categorization of the approximate 250 engineering student participants. A detailed examination of the overall study pool revealed the greatest numbers were in the unchanging groups (High-Static and Low-Static), yet the greatest potential for change is in the Low-Increasing groups. This suggests more effort needs to be made in engineering curricula to encourage students of lower motivation to increase as a result of their engineering experiences. These preliminary insights were also explored within the construction of two multinomial logistic regressions, which evaluated various additional hypotheses:

- The literature suggests a decrease in confidence has been correlated to an increase in anxiety level (Felder et al. 1995). This observation helped lead to forming a new hypothesis, that an increase in anxiety level would also impact engineering design motivation level, negatively. The logistic regression utilized anxiety levels, tabulated in a similar fashion to the engineering design motivation levels, averaging nine questions from Carberry's task-oriented instrument (Carberry et al. 2009; Carberry et al. 2010), to help support that an increase in anxiety should result in a decrease in motivation level.

- The exploration of success also suggested that "drive or motivation" affects confidence in success (Hutchison et al. 2006), which helped create a new hypothesis: students with higher self-reported levels of success are more motivated to do engineering design tasks. The logistic regression was also utilized to support this theory. It determined that, as scores for success increased students are more motivated to do engineering design tasks. 
- Some of the aforementioned studies help to link confidence and success to satisfaction (Felder et al. 1995), yet the Impacts of Service on Engineering Students survey additionally incorporates student incentive to participate as a means of measuring student satisfaction (Pierrakos et al. 2008a; Pierrakos et al. 2008b; Pierrakos et al. 2007). The suggestion that a greater sense of personal satisfaction is associated with students' self-reported level of success and incentive to complete engineering design tasks leads to higher engineering design motivation (Felder et al. 1995; Hutchison et al. 2006), was the hypothesis resulting from combining the literature review with the survey instrument. Additionally, the logistic regression was utilized in analyzing this hypothesis. Support for linking an increase in success and incentive to participate to engineering design motivation level resulted from the logistic regression, with one exception pertaining to students' incentive to participate in the engineering design task "recognition", or ability to identify what constitutes an engineering design task. One additional key takeaway point for student success noted that "support from faculty, experts or team members" was something that was linked to lower motivation, suggesting that as students increase the rate the level of support they get from faculty, experts or team members, they are more likely to be in a lower motivation category. This conclusion implies that the lower motivated students require a higher level of support in order to feel successful.

- The achievement goal theory developed into a five-factor framework addressing student self-reported levels of mastery avoidance and approach, performance avoidance and approach, as well as work avoidance (Ames 1992; Eccles et al. 1998; Elliot 1999; Pintrich 2000; Elliot and McGregor 2001; Pieper 2003; Finney et al. 2004; Miller 2008; Miller 2007). This was utilized in the Impacts of Service on Engineering Students' survey, as well in a sub-instrument, to address student attitudes (Finney et al. 2004). The following hypothesis resulted from the merging of the aforementioned information: students with higher levels of mastery and performance approach attitudes to do engineering design tasks will have higher levels of engineering design motivation than those with higher levels of avoidance attitudes. Although three of the five factors were inconclusive for the on-going longitudinal study on engineering students, the logistic regression did help to support that an increase in student self-assessed mastery approach to do engineering design work scores was linked to higher engineering design motivation, as well as an increase in work avoidance was linked to lower motivation.

- Gender was also evaluated in the logistic regression, yet returned inconclusive results to statistically support the claim that females are more motivated than males to do engineering design tasks. The hypothesis pertaining to females being more motivated showed some support from additional perspectives within the qualitative analysis. 
- Lastly the quantitative analysis of the Impacts of Service on Engineering Students study evaluated students' knowledge and skill levels as they related to motivation. The literature helped to suggest that confidence was influencing student levels of knowledge and skills (Besterfield-Sacre et al. 2001), which provided grounds for suggesting a greater level of knowledge and skills provides a basis for which students increase their motivation as a result of participating in engineering design programs (Besterfield-Sacre et al. 2001; Vallerand 2002; Areepattamannil et al 2011; Cech et al. 2011). The logistic regression was also utilized and helped determine that factors within knowledge and skills being labeled Personal Strengths and Future Plans contributed to an increase in motivation level, which helps to support the theory that an increase in knowledge and skills results in an increase in motivation.

\subsection{2 idesign Quantitative Conclusions}

Similar to the on-going longitudinal study on engineering students, a quantitative evaluation was conducted on Michigan Tech's idesign participants. Some findings of the aforementioned quantitative instruments were available outside of statistical analysis:

- The evaluation of the readiness to complete engineering design tasks assessment did not include a post assessment, which limited the findings to a threshold value of zero. Scores were above zero, which suggests preliminary readiness for the international project sojourn, however, post scores are needed to make any claims on program impacts.

- Intercultural competency was also evaluated for idesign participants and yielded a decrease in scores, which is inconclusive in supporting the associated hypothesis that increases in Intercultural Development Inventory scores will result in an increase level of engineering design motivation.

- Similar to the readiness scores, the motivation scores from the Impacts of Service on Engineering students lacked a post score for additional evaluation; however, initial scores are above the threshold value of 50, suggesting moderate to high levels of motivation prior to travel.

- Students' self-efficacy, beliefs, and interests related to sustainable engineering were evaluated using the Sustainable Engineering through Service Learning survey tool. This resulted in some increasing scores, which help to support an increase in motivation based on involvement in projects with a sustainability focus. Additionally, those students who failed to respond to the post survey had high preliminary scores, which may provide further support for the hypothesis associated with Sustainable Engineering through Service Learning.

- Lastly, gender was evaluated using all of the quantitative instruments to determine if differences were apparent from males to females. Readiness scores to perform engineering design tasks, Intercultural development inventory scores, and 
Sustainable Engineering through Service Learning scores (only post and attrition categories) all resulted in higher levels for females. This helps to support the hypothesis that females are more motivated to do engineering design tasks.

Future work, however, will be needed to statistically support any of the aforementioned claims. Due to the longitudinal nature of the study, the size of the participant pool was too small to be statistically analyzed. Further analysis was conducted qualitatively.

\subsubsection{PCMI Quantitative Conclusions}

Data from the Peace Corps Master's International participants was similarly limited, which also resulted in a predominantly qualitative analysis of some of the quantitative conclusions. The findings of those results are discussed below. However, some results of the quantitative instruments are available outside of statistical analysis:

- The literature supports the benefits of evaluating student preparedness, which lead to the formulation of the following hypothesis: Student who self-report higher levels of readiness to participate in their respective engineering program are more motivated with respect to engineering design tasks (Litzinger et al. 2005; Jesiek et al. 2011). The evaluation of the readiness to complete engineering design tasks assessment resulted in similar scores for pre and post assessment. Scores were above zero, which provides a promising base to help support an increase in readiness is related to an increase in motivation to do engineering design work. This could suggest a higher self-perceived level of readiness, which suggests those scoring as such are probably more motivated (or interested) in doing this kind of design work. Yet, further analysis and data collection is needed to effectively make this claim.

- Some of the aforementioned studies helped to suggest a need for evaluating intercultural understanding, which lead to the formulation of: Students with higher levels of intercultural competency are expected to have a better understanding of other cultures, leading them to be better equipped and more motivated to perform engineering design tasks in other societies (Hammer et al. 2003; Astin and Antonio 2004; Kuh and Umbach 2004). Intercultural competency was also evaluated for Peace Corps Master's International participants and yielded an increase in scores. This helps to support the associated hypothesis that increases in Intercultural Development Inventory scores should result in an increase level of engineering design motivation if cultural contexts and connections are made with respect to the design work since students are expressing a higher appreciation for the cultural context of design.

- The motivation scores from the Impacts of Service on Engineering students resulted in a decrease over time, which is inconclusive in supporting positive results associated with increasing engineering design motivations. This will continue to be evaluated as additional data is collected. 
- Explorations of sustainable engineering in previous studies lead to creating the following hypothesis: A better understanding of sustainable engineering should result in higher levels of student motivation to utilized these skills with regard to engineering design (Huntzinger et al. 2007; McCormick et al. 2010; McCormick et al. 2011). Similar to idesign, students' self-efficacy, beliefs, and interests related to sustainable engineering were evaluated using the Sustainable Engineering through Service Learning survey tool. Peace Corps Master's International students experienced some increasing scores, which help to support an increase in motivation based on a higher self-reported level of these aspects. Additionally, those students who failed to respond to the post survey had high preliminary scores, which may provide further support for the hypothesis associated with Sustainable Engineering through Service Learning.

- Lastly, various studies support the exploration of gender differences within engineering programs which helped create: Female students are more motivated to participate in engineering design programs when given the opportunity to apply what they are learning to help others (Felder et al. 1995; Brainard and Carlin 1997). Gender was evaluated using all of the quantitative instruments to determine if differences were apparent from males to females. Readiness scores to perform engineering design tasks, Intercultural development inventory scores (post and attrition categories), and Sustainable Engineering through Service Learning scores all resulted in higher levels for females. This helps to support the hypothesis that females are more motivated to do engineering design tasks.

Future work, however, will be needed to statistically support any of the aforementioned claims. Due to the longitudinal nature of the study, data will continue to be collected and re-evaluated to determine longitudinal tendencies for participants, which will help determine if these findings are consistent over time.

\subsection{Qualitative Methods Conclusions}

Chapter 2 outlined the qualitative methods utilized in evaluating the three Learning Through Service programs. The utilization of these methods is intended to support the aforementioned hypotheses (Chapter 1).

\subsubsection{ISES Qualitative Conclusions}

In addition to the survey, the on-going longitudinal study on engineering students also utilized interviews to better understand the participants. This resulted in qualitative information, which was evaluated using the aforementioned qualitative software HyperResearch $^{\circledR}$. The findings from this examination suggest students are motivated predominantly by five things (ties in percentages account for the additional two motivators): Interest/Enjoyment, Application of knowledge and skills, Knowledge, Personal significance, New perspective, Real world experience, Future circumstances, and New experiences. These motivations were found from 40 individuals who were interviewed three times for a total of 120 interviews. These same top five reasons were found overall, as well as for gender break down. One modification in the academic level breakdown, students with a higher level of education (juniors) did not suggest that future 
circumstances were a factor in motivating them, however, friends were additionally listed in their place. These findings help to begin to support qualitatively the following hypotheses: (1) female students are more motivated to participate in engineering design programs when given the opportunity to apply what they are learning to help others, (2a) a greater level of knowledge and skills provides a basis for which students increase their motivation as a result of participating in engineering design programs, and (7) individuals who have previous international experiences are more motivated to pursue others where they can additionally utilize their engineering design skills.

\subsection{2 idesign Qualitative Conclusions}

The idesign study additionally utilized qualitative analysis in the form of a word cloud and motivational narratives. Interviews are in the beta testing process and will be used in future qualitative analyses. Additionally the Readiness, Intercultural Development Inventory, Impacts of Service on Engineering Students' engineering design motivation, and the Sustainable Engineering through Service Learning scores were evaluated using qualitative observational assessment processes. The results of these instruments help to support the following (previously outlined in Chapter 1): (3) student who self-report higher levels of readiness to participate in their respective engineering program are more motivated with respect to engineering design tasks, (4) students with higher levels of intercultural competency are expected to have a better understanding of other cultures, leading them to be better equipped and more motivated to perform engineering design tasks in other societies, and (5) a better understanding of sustainable engineering should result in higher levels of student motivation to utilize these skills with regard to engineering design.

\subsubsection{PCMI Qualitative Conclusions}

Similar to the idesign program, a word cloud and motivational narratives were evaluated along with student Readiness, Intercultural Development Inventory, Impacts of Service on Engineering Students engineering design motivation, and the Sustainable Engineering through Service Learning scores utilizing qualitative analysis. These instruments also helped to support the following hypotheses (previously outlined in Chapter 1): (3) student who self-report higher levels of readiness to participate in their respective engineering program are more motivated with respect to engineering design tasks, (4) students with higher levels of intercultural competency are expected to have a better understanding of other cultures, leading them to be better equipped and more motivated to perform engineering design tasks in other societies, and (5) a better understanding of sustainable engineering should result in higher levels of student motivation to utilize these skills with regard to engineering design.

\subsection{Overall Conclusions}

The overall goal of this thesis was to determine what motivates students to pursue engineering design tasks. With the help of the three programs evaluated, a few general conclusions were found. Utilizing the on-going longitudinal study on engineering students, the logistic regression revealed that for students' self-reported level of mastery to do engineering design tasks, success and anxiety associated with completing engineering design tasks, satisfaction associated with performing engineering design 
tasks, the degree tasks would impact their level of being successful, as well as two factors associated with knowledge and skills (personal strengths and future plans) were found to impact students' engineering design motivation.

Additional examination excluding statistical analysis to suggest readiness to do engineering design tasks, intercultural competency, and understanding of sustainable engineering are also impacting student engineering design motivations. Commentary within the interviews and motivational narratives provided support for the aforementioned hypotheses related to these conclusions.

\subsection{Future Work}

Throughout this work, areas where additional work and evaluation were deemed necessary, were identified and discussed. This research would require additional time and resources to fully investigate and were therefore considered outside of the scope of this thesis project. Highlighted areas where additional work is suggested include the following:

- First and foremost, in order to completely evaluate these methodologies for purposes not limited to student engineering design motivations a larger study pool is crucial. In order to conduct statistical analysis on many of the aforementioned instruments the population sizes needed to be considerably larger. This could be accomplished with more time for single-program studies, or adding programs if understanding more general features are the interest.

- With respect to the on-going longitudinal study on engineering students, as previously mentioned, future work could be done to focus on trying to motivate students by improving their abilities to recognize engineering design tasks. As students feel more comfortable with this ability, they may be more motivated by it. This would require the development of appropriate learning materials and evaluating their impacts.

- Additionally, the logistic regression conducted on the on-going longitudinal study on engineering students suggested support from faculty, experts or team members was something that would likely place students in a low motivation group over a high. This conclusion suggests that students with a lower level of engineering design motivation require a higher level of support in order to feel successful. Future efforts should be put towards working to ensure students have good peer experiences, as well as having faculty who are supportive and available to help, could help to turn this question around for student motivation.

\subsection{Recommendations}

Although the conclusions and future work sections provide an understanding of these three on-going studies and what is needed to expand upon the existing results, they do no provide details on where to take the results next in future studies to continue to promote positive experiences and generally increase engineering design motivations. Below are 
some recommended questions to explore in future studies and what is needed to complete them.

\subsubsection{ISES Recommendations}

Examining the study pool available for the on-going longitudinal study on engineering students helps to demonstrate that there is a great potential for change in students who are in the Low-Increasing group. This study pool has very low numbers of individuals who fall in this category in comparison to Low-Static. The question of whether this is constant over time should be re-evaluated as the study continues and participants shift in motivation level. This will also help to determine whether the Low-Increasing group shifts to larger numbers of participants over time to help to suggest that students are increasing their motivation.

The logistic regression additionally helped in evaluating the engineering students within the on-going longitudinal study. Future studies should aim to determine if the same results found in this study are consistent over time. Additionally, exceptions were found in this study pool with respect to mean success, incentive to participate, as well as the degree an engineering task would affect students' level of being successful/unsuccessful. These need to be continually examined as the study continues to determine if this is consistent over time in participants or if this is just with respect to the included data. With respect to incentive, the exception was related to students' ability to recognize engineering design tasks. This could be utilized as a tool to help increase motivation. Courses utilizing assessment methodologies pre and post attendance that additionally focus on recognizing engineering design tasks throughout could help to determine if this is a legitimate claim. Future evaluations of these courses should utilize the question of whether students are able to recognize engineering design tasks. Gathering this data from various programs or courses will help to support or negate the claim that this is an exception to incentive related to satisfaction impacting motivation to do engineering design work.

Support from faculty, experts, team members, and peers were additionally noted as being an exception for students increasing levels of success from the logistic regression results. Universities should utilize this knowledge and make services available to students to help increase feelings of support, to increase perceptions of success, which is found to be correlated to levels of motivation within the logistic regression. Additionally, assessment methodologies should be utilized to determine if the available support services and faculty are sufficient from the students' perspective. The question of whether these available resources are increasing students' perceived levels of success associated with engineering design tasks or not, should be utilized. This exception should also be continually analyzed as the study continues to determine if this is consistent over time.

It was determined that mastery approach to engineering design tasks was statistically significant for students participating in the on-going longitudinal engineering study. However, additional factors associated with attitudes, like performance (approach and avoidance), as well as mastery avoidance were not deemed statistically significant. Evaluations need to continue to address these factors to help determine if this is 
consistent over time. Additional assessments could be utilized to determine if this differs from program to program through the use of the attitudes instrument, previously outlined in Chapter 2 (Finney et al. 2004). The hypothesis should assume these factors do impact motivation since the validated instrument demonstrates they have been in previous studies.

Knowledge and skills were evaluated using the logistic regression for the on-going longitudinal study on engineering students. Factors resulting from factor analysis were utilized to determine if knowledge and skills was directly impacting engineering design motivation. Factors of personal strengths and future plans were deemed statistically significant, however, Teaming, Improvement, Process recognition, Design process, Professional practice, Impact assessment, and Planning were not (Chapter 3). Future efforts for this study, as well as for similar studies, should continue to utilize these factors to determine if the results are consistent over time, as well as if the other factors become significant over time. Universities can utilize similar evaluative techniques that the Impacts of Service on Engineering Students survey used to examine their own programs for similar results.

Phone interviews were also utilized to gather additional information pertaining to student engineering design motivations. Future analysis of this data should examine what other tendencies are occurring in participants, as well as what tendencies are occurring in those participants that report leaving the engineering field while remaining in the study. These interview results can also be used in attracting and retaining female students, acknowledging that new experiences, future circumstances, and personal significance were noted factors that differed from male participants. Additionally, efforts should be put towards determining if these are also significant over time and if the factors impacting juniors over freshmen are consistent (real world experience, new perspective, new experience, and friends). Additional analysis is needed to determine if gender and education level differences can be deemed statistically significant, since the current studies were inconclusive with regard to statistical analysis from the logistic regression. The hypotheses should assume that females and those students with higher levels of education are more motivated to do engineering design work, thus data should be collected on a variety of levels of education, as well as for both male and female participants.

\subsection{2 idesign Recommendations}

Primary efforts for the on-going collection of the international senior design program should be on collecting data to enable statistical analysis of the independent variables. Additionally, the use of the formalized assessment now in place will aid in examining change over time, as some of the instruments lack a post score at this time.

The intercultural development inventory and Sustainable Engineering through Service Learning survey need further examination to determine if participants are consistently experiencing decreases in scores over time, as well as if female participants continue to score higher than males. Results of this will help to suggest whether better preparation is 
needed for participants involved in this program to help increase their intercultural awareness or sustainable engineering understanding prior to fieldwork.

Motivational scores resulting from the Impacts of Service on Engineering Students survey based off of Carberry's self-efficacy instrument (Carberry et al 2009; 2010) lack a post score, which is crucial in determining whether participants are changing over time or not. Additionally the participant pool size needs to increase in order for statistical analysis to be conducted to support increases in motivation levels.

Lastly, motivational narratives provide some in-depth information, which helps to support quantitative results; however, statistical analysis is lacking to determine if the quantitative results of the narratives are significant. Other universities should begin evaluating this information in their respective programs to determine if they experience gender, education level, or prior international experience level influences and if these results are consistent over time.

\subsubsection{PCMI Recommendations}

Similar to the international senior design program, the Peace Corps Master's International program needs to continue to increase the participant pool size that have completed survey instruments in order for statistical analysis to be an option. Additional evaluations of readiness to do engineering design work scores, Intercultural Development Inventory scores, motivation scores from the Impacts of Service on Engineering Students survey, as well as scores from the Sustainable Engineering through Service Learning survey need to be continually evaluated as more data comes available to determine if these results are consistent over time for the current participants, as well as if future participants differ in results. Universities can utilize these results to determine what is lacking in their programs that would aid in increasing motivation in participants. Having this extensive understanding of participants should increase retention and recruitment of students to these programs. 


\section{References}

Ames, Carole. 1992. Classrooms: Goals, Structures, and Student Motivation. Journal of Educational Psychology. 84(3):1-11.

Areepattamannil S, Freeman JG, Klinger DA. 2011. Intrinsic Motivation, Extrinsic Motivation, and Academic Achievement among Indian Adolescents in Canada and India. Social Psychology Education. 14: 427-39.

Astin HS, Antonio AL. 2004. The Impact of College on Character Development. New Directions for Institutional Research. Wiley Periodicals, Inc. 122:55-64.

Bennett MJ. 1993. Towards ethnorelativism: A developmental model of intercultural sensitivity. In: R. M. Paige (Ed.). Education for the intercultural experience, Intercultural Press, Yarmouth, ME. pp. 21-71.

Besterfield-Scare M, Moreno M, Shuman L, Atman C. 2001. Gender and ethnicity differences in freshmen engineering student attitudes: a cross-institutional study. Journal of Engineering Education. 90(4):477-489.

Bielefeldt AR, Paterson KG, Swan CW. 2010. Measuring the Value Added from Service Learning in Project-Based Engineering Education. International Journal of Engineering Education. In Special Issue on Problem-Based Learning. 26(3):1-12.

Brainard SG, Carlin L. 1998. A six-year longitudinal study of undergraduate women in engineering and science. Journal of Engineering Education. 87: 369-376.

Busch-Vishniac IJ, Jarosz JP. 2004. Can Diversity in the Undergraduate Engineering Population Be Enhanced Through Curricular Change? Journal of Women and Minorities in Science and Engineering. 10(3):50-77.

Boyer EL. 1996. The Scholarship of Engagement. Bulletin of the American Academy of Arts and Sciences. 49(7):18-33.

Carberry A, Ohland M, Lee H. 2009. Developing and instrument to Measure engineering design self-efficacy. American Society of Engineering Education.

Carberry, A., H. Lee, and M. Ohland (2010). Measuring engineering design self-efficacy. Journal of Engineering Education. 99:71-79

Cech E, Rubineau B, Silbey S, Seron C. 2011. Professional Role Confidence and Gendered Persistence in Engineering. American Sociological Review. 76(5):641666. 
Csikszentmihalyi, Mihaly. 1990. Flow: The Psychology of Optimal Experience. New York: Harper \& Row.

Eccles J, Wigfield A, Schiefele U. 1997. Motivation to Succeed. In: William Damon and Nancy Eisenberg. Handbook of Child Psychology. 5th ed.: Vol 3. Hoboken, NJ: John Wiley \& Son. p.1017-1095.

Eccles JS, Wigfield A. 2002. Motivational Beliefs, Values, and Goals. Annual Review of Psychology. 53 (1): 109-132.

Elliot AJ, McGregor HA. 2001. A 2x2 achievement goal framework. Journal of Personality and Social Psychology. 80:501-519.

Felder RM, Felder G, Mauney M, Hamrin JR. CE, Dietz EJ. 1995. A Longitudinal Study of Engineering Studetn Performance and Retention. Gender Differences in Student Performance and Attitudes. Journal of Engineering Education. 84(2), 151163.

Finney SJ, Pieper SL, Barron KE. 2004. Examining the Psychometric Properties of the Achievement Goal Questionnaire in a General Academic Context. Educational and Psychological Measurement. 64(2): 365-382.

Ford ME. 1992. Human Motivation: Goals, Emotions, and Personal Agency Beliefs. Newbury Park, CA: Sage Publications Ltd.

Gibbs G. 2007. Analyzing Qualitative Data. London: SAGE.

Grandin JM, Hirleman ED. 2009. Educating Engineers as Global Citizens: A Call for Action / A Report of the National Summit Meeting on the Globalization of Engineering Education. Online Journal of Global Engineering Education. 4(1): $10-25$.

Guzak K, Paterson KG. 2011. Learning Through Service Student Motivations, ASEE North Midwest Conference.

Guzak K, Paterson KG, Alexandra A. 2012. A Comparative Assessment of Graduate versus Undergraduate Student Outcomes via International Community Engagement Programs. ASEE Annual Conference.

Hammer MR, Bennett MJ, Wiseman R. 2003. Measuring intercultural sensitivity: The intercultural development inventory. International Journal of Intercultural Relations. 27(4): 421-443.

Huntzinger DN, Hutchins MJ, Gierke JS, Sutherland JW. 2007. Enabling Sustainable Thinking in Undergraduate Engineering Education. International Journal of Engineering Education. 23(2): 218-230. 
Hutchison MA, Follman DK, Sumpter M, Bodner GM. 2006. Factors Influencing the Self-Efficacy Beliefs of First-Year Engineering Students. Journal of Engineering Education. 95(1): 39-47.

Jesiek BK, Chang Y, Shen Y, Lin JJJ, Hirlemann ED, Groll EA. 2011. International Research and Education in Engineering (IREE) 2010 China: Developing Globally Competent Engineering Researchers. Proceedings of the American Society for Engineering Education Annual Conference. Vancouver, BC. AC2011-1975. 14 pp.

Jordan KL, Amato-Henderson, S, Sorby SA, Haut Donahue TL. 2011. Are there differences in self-efficacy between minority and majority students across academic levels? Proceedings of the American Society for Engineering Education Annual Conference. Vancouver, BC. AC2011-659. 13 pp.

Katehi L. 2005. The Global Engineer. In Educating the Engineer of 2020: Adapting Engineering Education to the New Century. The National Academies Press, Washington, DC. pp. 151-155.

Keyes CLM. 2006. Flourishing. American Journal of Orthopsychiatry.

Kuh GD, Umbach PD. 2004. College and Character: Insights from the National Survey of Student Engagement. New Directions for Institutional Research. Wiley Periodicals, Inc. 122:37-54.

Kvale S, Brinkmann S. 2009. Interviews: Learning the Craft of Qualitative Research Interviewing. Sage Publications, Thousand Oaks, California.

LeCompte MD, Schensul JJ. 1999. Analyzing \& Interpreting Ethnographic Data. Walnut Creek: Altamira.

Lindlof TR, Taylor BC. 2011. Qualitative Communication Research Methods. Thousand Oaks, CA: SAGE.

Matusovich H, Streveler R, Miller R. 2009. What Does 'Motivation' Really Mean? an Example From Current Engineering Education Research. (June 13): 1-7.

McCormick M, Lawyer K, Berlin M, Swan C, Paterson K, Bielefeldt A, Wiggins J. 2010. Evaluation of Sustainable Engineering Education via Service Learning and Community Service Efforts. Proceedings of the American Society for Engineering Education Annual Conference. Louisville, KY. 
McCormick M, Swan C, Bielefeldt A, Lawyer K, Paterson K. 2011. Service Learning: Motivating students to engineer sustainably. Proceedings of the American Society for Engineering Education Annual Conference. Vancouver, BC. AC2011-1335.

Miller BJ. 2007. Achievement Goal Orientation Toward General Education vs. Overall Coursework. General Education Coursework. (October 25): 1-13.

National Academy of Engineering. 2008. Changing the Conversation: Messages for Improving Public Understanding of Engineering. Committee on the Public Understanding of Engineering Messages. National Academies Press, Washington, DC. pp. 149

National Science Board. 2008. Science and Engineering Indicators. National Science Foundation, Arlington, VA. (1 and 2): 1164 .

National Science Foundation. 2011. Women, Minorities, and Persons with Disabilities in Science and Engineering: 2011. National Science Foundation, Arlington, VA. pp. 16

Nicholls JG, Cobb P, Yackel E, Wood T, Wheatley G. 1990. Students' theories of mathematics and their mathematical knowledge: Multiple dimensions of assessment. In G. Kulm (Ed.), Assessing higher order thinking in mathematics. Washington, DC: American Association for the Advancement of Science. pp. $137-154$

Parkinson A. 2007. Engineering Study Abroad Programs: Formats, Challenges, Best Practices. Global Journal of Engineering Education. 2(2): 1-15.

Paterson KG, Fuchs VJ. 2008. Development for the Other 80\%: Engineering Hope. Australasian Journal of Engineering Education. 14(1):1-12.

Paterson K, O'Holleran C, Leslie C. 2010. Faculty Impressions of Service Learning in Engineering Education. Proceedings of the American Society for Engineering Education Annual Conference. Louisville, KY. AC2010-2033, 8 pp.

Pieper SL. 2003. Refining and Extending the 2 x 2 Achievement Goal Framework: Another Look at Work-Avoidance. Ph.D. Dissertation, James Madison University.

Pierrakos O, Lo J, Borrego M. 2007. Assessing Learning Outcomes of Senior Mechanical Engineers in a Capstone Design Experience, American Society for Engineering Education (ASEE) Conference and Exposition Proceedings, Honolulu, Hawaii. 
Pierrakos O, Borrego M, Lo J. 2008a. Assessing Learning Outcomes During Summer Undergraduate Research Experiences, American Society for Engineering Education (ASEE) Conference and Exposition Pittsburg, PA.

Pierrakos O, Borrego M, Lo J. 2008b. Preliminary Findings from a Quantitative Study; What Are Students Learning During Cooperative Education Experiences? American Society for Engineering Education (ASEE) Conference and Exposition Pittsburg, PA.

ResearchWare, Inc. 2010. HyperRESEARCH User Guide, ver.3. Randolph, MA. 171 pp.

Saldaña J. 2009. The Coding Manual for Qualitative Researchers. London: Sage.

Seymour E, Hewitt N. 2000. Talking About Leaving: Why Undergraduates leave the Sciences. Westview Press, Boulder, Colorado. 444 pp.

Sheppard S, Gilmartin S, Chen HL, Donaldson K, Lichtenstein G, Eris Ö, Lande M, Toye G. 2010. Exploring the Engineering Student Experience: Findings from the Academic Pathways of People Learning Engineering Survey (APPLES) (TR-1001). Seattle, WA: Center for the Advancement for Engineering Education.

Sinclair J, Cardow-Hall M. 2008. The folksonomy tag cloud: when is it useful? Journal of Information Science. 3(15): 15-29.

Sigma Xi. 2007. Embracing Globalization: Assuring a Globally Engaged Science and Engineering Workforce (Workshop Report). Sigma Xi, Washington, DC.

Silliman S, Mohtar R, Paterson KG, Ball W. 2010. Engineering Academic Programs for Hydrophilanthropy: Commonalities and Challenges. Journal of Contemporary Water Research and Education. 145:5-29.

Tinto V. 1994. Leaving College: Rethinking the causes and cures of student attrition. University of Chicago Press. 312 pp.

Vallerand RJ. 2002. Intrinsic and Extrinsic Motivation: A Hierarchical Model. Handbook of Self-determination Research. Deci, EL, Ryan RM. Rochester, NY: University of Rochester. pp 281.

Vest CM. 2006. Educating Engineers for 2020 and Beyond. The Bridge: Linking Engineering and Society. National Academy of Engineering. 36(2):38-44 


\section{Appendix: Quantitative Assessment Instrument Samples}

\begin{tabular}{|c|c|c|c|c|c|c|}
\hline \multirow[b]{2}{*}{ Readiness Assessment } & & \multicolumn{5}{|c|}{$\begin{array}{l}\text { Last four digits of your cell phone number? } \\
\text { (only used for tracking assessment data/results) }\end{array}$} \\
\hline & & $-\ldots$ & $--\ldots$ & & & \\
\hline Please evaluate each statement listed below: & $\begin{array}{l}\text { Strongly } \\
\text { disagree }\end{array}$ & Disagree & $\begin{array}{c}\text { Disagree } \\
\text { a little bit }\end{array}$ & $\begin{array}{l}\text { Agree a } \\
\text { little bit }\end{array}$ & Agree & $\begin{array}{c}\text { Strongly } \\
\text { agree }\end{array}$ \\
\hline 1. I am anxious about going abroad. & $\square$ & $\square$ & $\square$ & $\square$ & $\square$ & $\square$ \\
\hline 2. If I need help while abroad, I will know who to contact. & $\square$ & $\square$ & $\square$ & $\square$ & $\square$ & $\square$ \\
\hline 3. While abroad, I know how to keep in touch with my family. & $\square$ & $\square$ & $\square$ & $\square$ & $\square$ & $\square$ \\
\hline 4. I am prepared to go abroad. & $\square$ & $\square$ & $\square$ & $\square$ & $\square$ & $\square$ \\
\hline 5. I question if going abroad was a good decision. & $\square$ & $\square$ & $\square$ & $\square$ & $\square$ & $\square$ \\
\hline 6. I can deal with unexpected challenges while abroad. & $\square$ & $\square$ & $\square$ & $\square$ & $\square$ & $\square$ \\
\hline
\end{tabular}

Figure 7.1: Readiness assessment inventory (Jesiek et al. 2011) sample questions

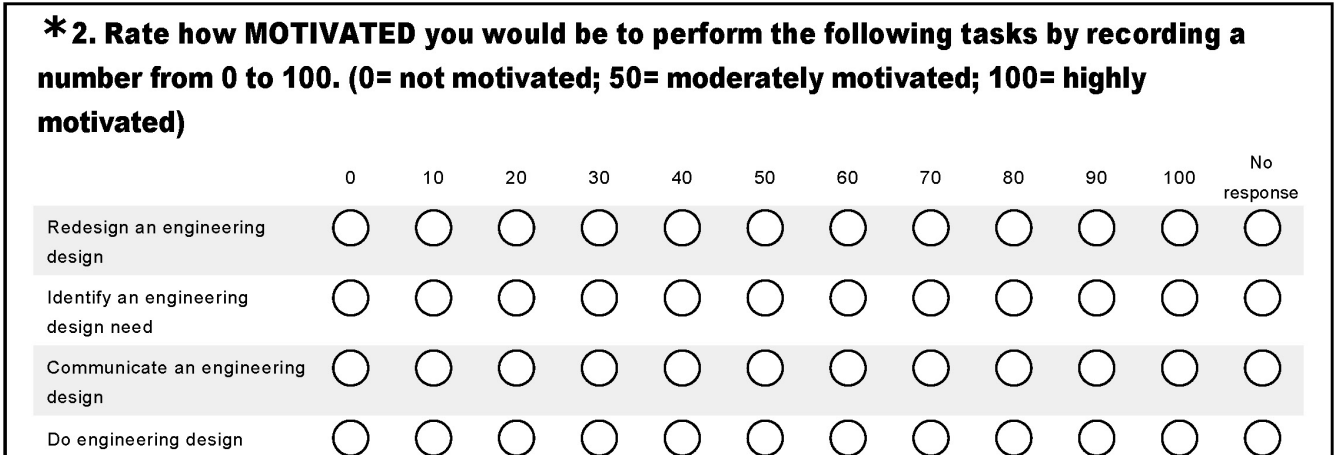

Figure 7.2: ISES survey sample questions (Pierrakos et al. 2008a; Pierrakos et al. 2008b; Pierrakos et al. 2007)

Please answer all of the following questions fully by selecting the answer that best represents your beliefs and judgment of your current abilities. Answer each question in terms of who you are and what you know today about the given tasks.

* Please rate your degree of confidence to perform the following on a scale of 0 to $100(0=$ no confidence; $\mathbf{5 0}$ = moderately confident; 100 = very confident)

Identify the environmental elements of an engineering project.

Know how environmental systems work.

Understand environmental risks associated with engineering projects.

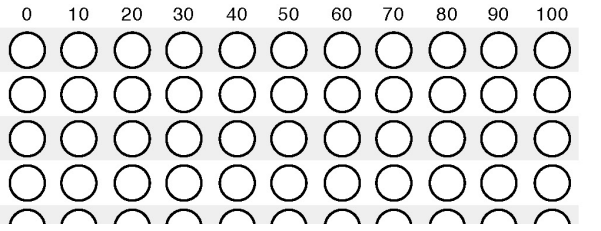

Figure 7.3: SESL survey sample questions (McCormick et al. 2010; McCormick et al. 2011) 
7.1 Motivational narrative prompt

\section{Task: Write a narrative, no more than one page at 12 point font, describing your motivations for wanting to participate in the (idesign/PCMI) program.}

(Guzak and Paterson 2011; Guzak et al. 2012)

\subsection{Sample ISES interview questions}

Background

-Where are you currently a student?

-What are you majoring in?

- Have you changed majors (since we last spoke)?

-(If YES) What attracted you to that major?

-(If NO) Are you more or less (or the same) interested in your major since starting?

- Have recent national/global economic conditions influenced your choice in major? If so, how?

\section{Attitude}

- Have you ever thought about changing majors or dropping out of school? What's motivated you to stay (or change)?

-What do you see yourself contributing through your chosen profession?

-On a more regular (day-to-day) basis what motivates you to learn (or stay in) this field?

-Does your university meet your educational needs? How can it improve? 


\section{Appendix: Motivational Narrative Codes}

Table 8.1

Motivational narrative codes and definitions

\begin{tabular}{|l|l|}
\hline Code Name & Definition \\
\hline Career goal & $\begin{array}{l}\text { Personal ambition, international experience will help } \\
\text { with resume or other forms of career aspirations }\end{array}$ \\
\hline Class influence & $\begin{array}{l}\text { Within a class it was suggested that international } \\
\text { experiences are beneficial }\end{array}$ \\
\hline Community need & $\begin{array}{l}\text { A desire to work with people to get them what they } \\
\text { need as opposed to giving them what is thought they } \\
\text { need }\end{array}$ \\
\hline Desire to work abroad & $\begin{array}{l}\text { Personal ambition to work outside of the United } \\
\text { States }\end{array}$ \\
\hline $\begin{array}{l}\text { Desires unconventional } \\
\text { job }\end{array}$ & $\begin{array}{l}\text { Personal ambition to work in a setting that is } \\
\text { atypical of the engineering } 9 \text { to 5 job }\end{array}$ \\
\hline Efficient aid & $\begin{array}{l}\text { A desire to work with the people to get them what } \\
\text { they need even if this means not personally traveling }\end{array}$ \\
\hline Family influence & $\begin{array}{l}\text { Family members suggest the importance of } \\
\text { international experiences }\end{array}$ \\
\hline Hands on experience & $\begin{array}{l}\text { A desire to use the material learned in class out in } \\
\text { the field to solve real problems }\end{array}$ \\
\hline Helping others & A desire to assist people other than oneself \\
\hline Mentor & $\begin{array}{l}\text { An outside source like a professor or advisor } \\
\text { suggests that international experiences are useful }\end{array}$ \\
\hline New opportunity & $\begin{array}{l}\text { The prospect of experiencing something outside the } \\
\text { ordinary }\end{array}$ \\
\hline Individual ambition to do something internationally \\
\hline Personal goal & $\begin{array}{l}\text { Individual problems one must overcome while } \\
\text { working internationally }\end{array}$ \\
\hline Personal struggle & $\begin{array}{l}\text { A religious belief impacts the desire to work abroad } \\
\text { through the desire to help, teach, learn, etc. }\end{array}$ \\
\hline Religion & $\begin{array}{l}\text { A desire to work with circumstances to overcome } \\
\text { obstacles others face }\end{array}$ \\
\hline Solving problems & $\begin{array}{l}\text { Trying to find some sense of purpose, meaning and } \\
\text { structure to life }\end{array}$ \\
\hline Spirituality & $\begin{array}{l}\text { The reputation of the international program precedes } \\
\text { itself, encouraging students to participate }\end{array}$ \\
\hline Successful reputation & The desire to give one's time \\
\hline Working with people & $\begin{array}{l}\text { The desire to work with others to reach a common } \\
\text { goal and learn from each other }\end{array}$ \\
\hline
\end{tabular}




\section{Appendix: ISES SPSS ${ }^{\circledR}$ Factor Analysis Results}

\subsection{Knowledge and Skills Part 1 Factor Analysis}

Table 9.1

Knowledge and skills part 1 factor analysis rotated component matrix

Rotated Component Matrix ${ }^{a}$

\begin{tabular}{|l|l|l|l|l|}
\hline & \multicolumn{4}{|c|}{ Component } \\
\cline { 2 - 5 } & 1 & \multicolumn{1}{|c|}{2} & 3 & \multicolumn{1}{l|}{4} \\
\hline KSQ1.11 & .789 & .156 & .211 & .163 \\
KSQ1.10 & .735 & .190 & .220 & .215 \\
KSQ1.12 & .720 & .129 & .405 & .086 \\
KSQ1.9 & .702 & .184 & .163 & .216 \\
KSQ1.13 & .630 & .219 & .307 & .038 \\
KSQ1.8 & .547 & .536 & .101 & .082 \\
KSQ1.1 & -.016 & .737 & .331 & .197 \\
KSQ1.2 & .100 & .697 & .374 & .067 \\
KSQ1.14 & .254 & .692 & .119 & .086 \\
KSQ1.3 & .192 & .595 & .205 & -.005 \\
KSQ1.5 & .269 & .576 & .053 & .263 \\
KSQ1.7 & .481 & .501 & .029 & .021 \\
KSQ1.4 & .460 & .464 & .074 & .228 \\
KSQ1.6 & .201 & .455 & .077 & .345 \\
KSQ1.16 & .369 & .057 & .771 & .153 \\
KSQ1.17 & .329 & .102 & .743 & .145 \\
KSQ1.15 & .216 & .284 & .693 & .004 \\
KSQ1.18 & .147 & .319 & .651 & .205 \\
KSQ1.19 & .052 & .403 & .524 & .368 \\
KSQ1.21 & .154 & .106 & .146 & .786 \\
KSQ1.20 & .244 & .198 & .205 & .774 \\
\hline
\end{tabular}

Extraction Method: Principal Component Analysis. Rotation Method: Varimax with Kaiser Normalization.

a. Rotation converged in 9 iterations. 
Table 9.2

Knowledge and skills part 1 factor analysis total variance explained

Total Variance Explained

\begin{tabular}{|c|c|c|c|c|c|c|c|}
\hline \multirow[b]{2}{*}{ Component } & \multicolumn{3}{|c|}{ Initial Eigenvalues } & \multicolumn{3}{|c|}{ Extraction Sums of Squared Loadings } & \multirow{2}{*}{$\frac{\text { Rotation }}{\text { Total }}$} \\
\hline & Total & $\%$ of Variance & Cumulative \% & Total & $\%$ of Variance & Cumulative \% & \\
\hline 1 & 8.449 & 40.234 & 40.234 & 8.449 & 40.234 & 40.234 & 3.936 \\
\hline 2 & 1.622 & 7.724 & 47.957 & 1.622 & 7.724 & 47.957 & 3.724 \\
\hline 3 & 1.425 & 6.787 & 54.744 & 1.425 & 6.787 & 54.744 & 3.099 \\
\hline 4 & 1.127 & 5.367 & 60.111 & 1.127 & 5.367 & 60.111 & 1.865 \\
\hline 5 & .934 & 4.449 & 64.560 & & & & \\
\hline 6 & .852 & 4.057 & 68.617 & & & & \\
\hline 7 & .799 & 3.805 & 72.422 & & & & \\
\hline 8 & .697 & 3.320 & 75.743 & & & & \\
\hline 9 & .627 & 2.988 & 78.730 & & & & \\
\hline 10 & .584 & 2.782 & 81.513 & & & & \\
\hline 11 & .526 & 2.503 & 84.016 & & & & \\
\hline 12 & .471 & 2.244 & 86.260 & & & & \\
\hline 13 & .439 & 2.088 & 88.348 & & & & \\
\hline 14 & .401 & 1.910 & 90.258 & & & & \\
\hline 15 & .386 & 1.837 & 92.095 & & & & \\
\hline 16 & .362 & 1.723 & 93.818 & & & & \\
\hline 17 & .324 & 1.543 & 95.362 & & & & \\
\hline 18 & .300 & 1.427 & 96.789 & & & & \\
\hline 19 & .254 & 1.209 & 97.998 & & & & \\
\hline 20 & .217 & 1.035 & 99.033 & & & & \\
\hline 21 & .203 & .967 & 100.000 & & & & \\
\hline
\end{tabular}




\subsection{Knowledge and Skills Part 2 Factor Analysis}

Table 9.3

Knowledge and skills part 2 factor analysis rotated component matrix

Rotated Component Matrix ${ }^{a}$

\begin{tabular}{|l|l|l|l|l|l|}
\hline & \multicolumn{5}{|c|}{ Component } \\
\cline { 2 - 6 } & 1 & 2 & 3 & 4 & \multicolumn{1}{l|}{5} \\
\hline KSQ2.9 & .772 & .123 & .085 & .240 & .160 \\
KSQ2.12 & .669 & .134 & .061 & .305 & .116 \\
KSQ2.8 & .608 & .325 & .350 & -.005 & .224 \\
KSQ2.7 & .588 & .044 & .116 & .346 & .060 \\
KSQ2.13 & .576 & .280 & .379 & .194 & .240 \\
KSQ2.10 & .575 & .422 & .220 & .148 & .217 \\
KSQ2.11 & .571 & .209 & .148 & .483 & -.019 \\
KSQ2.3 & .566 & .303 & .172 & .115 & .232 \\
KSQ2.25 & .525 & .162 & .225 & .180 & .204 \\
KSQ2.27 & .489 & .182 & .415 & .360 & .193 \\
KSQ2.5 & .207 & .788 & .095 & .094 & .169 \\
KSQ2.6 & .194 & .680 & .254 & .299 & .156 \\
KSQ2.4 & .307 & .634 & .144 & .161 & .313 \\
KSQ2.1 & .128 & .587 & .407 & .200 & .171 \\
KSQ2.16 & .202 & .540 & .387 & .108 & .140 \\
KSQ2.2 & .494 & .497 & .366 & .064 & .083 \\
KSQ2.18 & .232 & .128 & .806 & .158 & .044 \\
KSQ2.17 & .212 & .288 & .773 & .081 & .031 \\
KSQ2.20 & .229 & .374 & .651 & .186 & .241 \\
KSQ2.21 & .073 & .351 & .561 & .223 & .341 \\
KSQ2.30 & .161 & .118 & .049 & .784 & .243 \\
KSQ2.28 & .354 & .195 & .133 & .743 & .119 \\
KSQ2.29 & .428 & .278 & .203 & .638 & .081 \\
KSQ2.26 & .335 & .087 & .271 & .627 & .204 \\
KSQ2.22 & .135 & .412 & .378 & .498 & .348 \\
KSQ2.23 & .095 & .265 & .051 & .142 & .788 \\
KSQ2.14 & .151 & .296 & .027 & .118 & .765 \\
KSQ2.24 & .319 & .007 & .313 & .190 & .695 \\
KSQ2.15 & .278 & .425 & .215 & .240 & .526 \\
KSQ2.19 & .351 & .034 & .466 & .174 & .485 \\
\hline
\end{tabular}

Extraction Method: Principal Component Analysis.

Rotation Method: Varimax with Kaiser Normalization.

a. Rotation converged in 8 iterations. 
Table 9.4

Knowledge and skills part 2 factor analysis total variance explained

Total Variance Explained

\begin{tabular}{|c|c|c|c|c|c|c|c|}
\hline \multirow[b]{2}{*}{ Component } & \multicolumn{3}{|c|}{ Initial Eigenvalues } & \multicolumn{3}{|c|}{ Extraction Sums of Squared Loadings } & \multirow{2}{*}{$\frac{\text { Rotation }}{\text { Total }}$} \\
\hline & Total & $\%$ of Variance & Cumulative \% & Total & $\%$ of Variance & Cumulative \% & \\
\hline 1 & 13.454 & 44.847 & 44.847 & 13.454 & 44.847 & 44.847 & 5.014 \\
\hline 2 & 1.978 & 6.592 & 51.439 & 1.978 & 6.592 & 51.439 & 3.970 \\
\hline 3 & 1.566 & 5.219 & 56.658 & 1.566 & 5.219 & 56.658 & 3.782 \\
\hline 4 & 1.190 & 3.966 & 60.624 & 1.190 & 3.966 & 60.624 & 3.408 \\
\hline 5 & 1.146 & 3.819 & 64.442 & 1.146 & 3.819 & 64.442 & 3.158 \\
\hline 6 & .915 & 3.050 & 67.492 & & & & \\
\hline 7 & .874 & 2.912 & 70.404 & & & & \\
\hline 8 & .734 & 2.447 & 72.851 & & & & \\
\hline 9 & .678 & 2.259 & 75.109 & & & & \\
\hline 10 & .657 & 2.189 & 77.299 & & & & \\
\hline 11 & .636 & 2.121 & 79.420 & & & & \\
\hline 12 & .524 & 1.748 & 81.168 & & & & \\
\hline 13 & .505 & 1.683 & 82.851 & & & & \\
\hline 14 & .479 & 1.598 & 84.449 & & & & \\
\hline 15 & .470 & 1.568 & 86.017 & & & & \\
\hline 16 & .429 & 1.431 & 87.449 & & & & \\
\hline 17 & .406 & 1.353 & 88.802 & & & & \\
\hline 18 & .369 & 1.230 & 90.032 & & & & \\
\hline 19 & .347 & 1.156 & 91.188 & & & & \\
\hline 20 & .337 & 1.125 & 92.313 & & & & \\
\hline 21 & .320 & 1.066 & 93.379 & & & & \\
\hline 22 & .283 & .944 & 94.323 & & & & \\
\hline 23 & .264 & .879 & 95.202 & & & & \\
\hline 24 & .249 & .829 & 96.031 & & & & \\
\hline 25 & .233 & .777 & 96.808 & & & & \\
\hline 26 & .211 & .702 & 97.509 & & & & \\
\hline 27 & .210 & .700 & 98.209 & & & & \\
\hline 28 & .188 & .625 & 98.834 & & & & \\
\hline 29 & .180 & .600 & 99.434 & & & & \\
\hline 30 & .170 & .566 & 100.000 & & & & \\
\hline
\end{tabular}




\subsection{Attitudes Factor Analysis}

Table 9.5

Attitudes factor analysis rotated component matrix

Rotated Component Matrix ${ }^{a}$

\begin{tabular}{|l|r|r|r|r|r|}
\hline & \multicolumn{5}{|c|}{ Component } \\
\cline { 2 - 6 } & \multicolumn{1}{|c|}{1} & \multicolumn{1}{|c|}{2} & \multicolumn{1}{c|}{3} & \multicolumn{1}{c|}{4} & \multicolumn{1}{c|}{5} \\
\hline A10 & .863 & -.121 & -.050 & .033 & -.025 \\
A3 & .824 & -.111 & .134 & .032 & .010 \\
A7 & .797 & -.176 & .167 & .031 & -.028 \\
A16 & .648 & -.308 & .189 & -.048 & .016 \\
A9 & -.160 & .894 & .044 & -.013 & .058 \\
A13 & -.242 & .859 & .085 & -.052 & .079 \\
A4 & -.221 & .850 & -.034 & .097 & .109 \\
A12 & .106 & .007 & .876 & .076 & .120 \\
A6 & .093 & .082 & .866 & .009 & .165 \\
A1 & .167 & -.015 & .854 & -.037 & .109 \\
A14 & .008 & .023 & -.014 & .890 & .021 \\
A11 & .123 & -.031 & .085 & .850 & .138 \\
A5 & -.070 & .037 & -.021 & .832 & .091 \\
A15 & -.015 & .101 & .340 & .005 & .775 \\
A2 & .005 & .235 & .006 & .068 & .763 \\
A8 & -.020 & -.079 & .111 & .164 & .717 \\
\hline
\end{tabular}

Extraction Method: Principal Component Analysis.

Rotation Method: Varimax with Kaiser Normalization.

a. Rotation converged in 6 iterations. 
Table 9.6

Attitudes factor analysis total variance explained

Total Variance Explained

\begin{tabular}{|l|r|r|r|r|r|r|r|}
\hline \multirow{2}{*}{ Component } & \multicolumn{3}{|c|}{ Initial Eigenvalues } & \multicolumn{2}{c|}{ Extraction Sums of Squared Loadings } & Rotation . \\
\cline { 2 - 8 } & Total & \% of Variance & Cumulative \% & Total & \% of Variance & Cumulative \% & Total \\
\hline 1 & 3.806 & 23.790 & 23.790 & 3.806 & 23.790 & 23.790 & 2.682 \\
3 & 3.185 & 19.906 & 43.696 & 3.185 & 19.906 & 43.696 & 2.494 \\
4 & 2.226 & 13.914 & 57.610 & 2.226 & 13.914 & 57.610 & 2.476 \\
5 & 1.320 & 8.252 & 65.862 & 1.320 & 8.252 & 65.862 & 2.262 \\
6 & 1.177 & 7.358 & 73.221 & 1.177 & 7.358 & 73.221 & 1.801 \\
7 & .734 & 4.587 & 77.808 & & & \\
8 & .562 & 3.514 & 81.322 & & & & \\
9 & .510 & 3.186 & 84.508 & & & & \\
10 & .439 & 2.743 & 87.251 & & & & \\
11 & .394 & 2.464 & 89.715 & & & & \\
12 & .340 & 2.124 & 91.839 & & & & \\
13 & .323 & 2.018 & 93.858 & & & & \\
14 & .291 & 1.820 & 95.678 & & & & \\
15 & .270 & 1.690 & 97.368 & & & & \\
16 & .222 & 1.390 & 98.757 & & & & \\
\end{tabular}




\section{Appendix: ISES Data Processing}

Prior to being able to analyze and utilize the ISES data it was necessary to process the various instruments' output. The remainder of this section outlines how these instruments were processed for further analysis.

\subsection{Online Surveys}

The approximate 250 student participants complete these online surveys using a given link to SurveyMonkey ${ }^{\circledR}$, which tabulates all participants and can be exported in a variety of ways. This data is generally exported using the comma separated values (CSV) option with the actual values instead of the numerical values. These files are then edited to shorthand the questions for use in SPSS ${ }^{\circledR}$. Nominal numerical values are assigned to student responses, additionally responses that were left blank or not applicable (e.g. grad students submitting survey responses in Spring 2011 were omitted) were removed for ease in analysis. An additional step of filtering out the interviewees and creating a separate file with only their information was added using the statistical software $\mathrm{R}$ for additional analysis, which was used to separate these participants from the overall pool. The formatted file containing all participants was then imported into SPSS ${ }^{\circledR}$ for further evaluation.

Because of the large amount of data available, factor analysis was conducted on some of the instruments within the survey to reduce the dimensionality of the data. The Knowledge and Skills parts one and two, as well as the Attitudes sections were factorreduced using factor analysis using a Varimax rotation within SPSS $^{\circledR}$. This resulted in four and five factors for the Knowledge and Skills sections and five factors for the Attitudes section, thus reducing the number of independent variables in the remaining analyses (Appendix 9). Future work will cover the naming and use of these fourteen factors.

\subsection{Interviews}

The information from 121, 110, and 98 students who completed interviews in Spring 2011, Fall 2011, and Spring 2012, respectively were processed in two mildly different manners. Spring 2011 data was transcribed solely using HyperTranscribe ${ }^{\circledR}$ and coded using HyperResearch ${ }^{\circledR}$. This procedure required that the transcriber listen to the interview and type verbatim what was said (Kvale and Brinkmann 2009). HyperTranscribe ${ }^{\circledR}$ enabled the listener to repeat segments of the interview to assist in understanding what was said. Each interview would take anywhere between 2 and 7 hours to transcribe using this method, which added the necessity of incorporating more assistance in this step. Five transcribers were utilized in this phase of data processing. For Fall 2011 and Spring 2012 data, however, Dragon Dictate ${ }^{\circledR}$ was incorporated to expedite the process reducing the necessity of multiple transcribers. Dragon Dictate ${ }^{\circledR}$ merged with HyperTranscribe ${ }^{\circledR}$ enabled the transcriber to listen to the interview and repeat what was said into a microphone. Dragon Dictate ${ }^{\circledR}$ would then transcribe what was said with some degree of accuracy. HyperTranscribe ${ }^{\circledR}$ would still enable the repetition of segments, which when merged with Dragon Dictate ${ }^{\circledR}$ accelerated the process. Unfortunately, Dragon Dictate ${ }^{\circledR}$ could only be trained well to one voice at a time, which limited the number of 
transcribers. This method, however, expedited the process to approximately 30 minutes to 1 hour. Once interviews were converted to a text format they were imported into HyperResearch ${ }^{\circledR}$ where they were then coded using the aforementioned thematic in vivo coding process. The results section depicts the frequency reports and findings of this step. 


\section{Appendix: Logistic Regression Results and Tables}

Table 11.1

Logistic regression with High-Static as a reference category with grey highlighting the significant independent variables

\begin{tabular}{|c|c|c|c|c|c|}
\hline IV Code & IV Description & Reference & Beta & Significance & Target \\
\hline AF2_WAV & Work Avoidance & H-S & 2.009 & 0.085 & H-D \\
\hline AF2_WAV & & $\mathrm{H}-\mathrm{S}$ & 0.943 & 0.787 & L-S \\
\hline AF2_WAV & & $\mathrm{H}-\mathrm{S}$ & 0.778 & 0.596 & L-I \\
\hline Class $=1$ & Freshmen & $\mathrm{H}-\mathrm{S}$ & 4.231 & 0.259 & H-D \\
\hline Class $=1$ & & $\mathrm{H}-\mathrm{S}$ & 2.022 & 0.486 & L-I \\
\hline Class $=1$ & & $\mathrm{H}-\mathrm{S}$ & 1.895 & 0.273 & L-S \\
\hline Class $=2$ & Junior & $\mathrm{H}-\mathrm{S}$ & 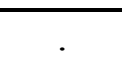 & . & H-D \\
\hline Class $=2$ & & $\mathrm{H}-\mathrm{S}$ & & . & L-I \\
\hline Class $=2$ & & $\mathrm{H}-\mathrm{S}$ & & & L-S \\
\hline ESM & Success Mean & H-S & 0.953 & 0.026 & L-S \\
\hline ESM & & H-S & 0.943 & 0.086 & H-D \\
\hline ESM & & H-S & 0.921 & 0.003 & L-I \\
\hline Gender $=0$ & Male & $\mathrm{H}-\mathrm{S}$ & 2.044 & 0.552 & H-D \\
\hline Gender $=0$ & & H-S & 0.422 & 0.147 & L-S \\
\hline Gender $=0$ & & H-S & 0.108 & 0.022 & L-I \\
\hline Gender $=1$ & Female & $\mathrm{H}-\mathrm{S}$ & . & . & H-D \\
\hline Gender $=1$ & & H-S & . & . & L-I \\
\hline Gender $=1$ & & H-S & & & L-S \\
\hline INC. 2 & $\begin{array}{l}\text { Incentive to participate: } \\
\text { "Help society or solve a } \\
\text { societal need" }\end{array}$ & $\mathrm{H}-\mathrm{S}$ & 1.051 & 0.427 & H-D \\
\hline INC.2 & & $\mathrm{H}-\mathrm{S}$ & 0.998 & 0.947 & L-I \\
\hline INC.2 & & H-S & 0.951 & 0.021 & L-S \\
\hline INC.4 & $\begin{array}{l}\text { Incentive to participate: } \\
\text { "Learn something new } \\
\text { and/or gain experience" }\end{array}$ & $\mathrm{H}-\mathrm{S}$ & 0.952 & 0.303 & H-D \\
\hline INC.4 & & H-S & 0.947 & 0.039 & L-S \\
\hline INC.4 & & H-S & 0.887 & 0.003 & L-I \\
\hline INC. 8 & $\begin{array}{l}\text { Incentive to participate: } \\
\text { "Presents a challenge" }\end{array}$ & $\mathrm{H}-\mathrm{S}$ & 1.004 & 0.894 & H-D \\
\hline INC. 8 & & $\mathrm{H}-\mathrm{S}$ & 0.996 & 0.84 & L-I \\
\hline
\end{tabular}


Table 11.2

Continuation of Logistic regression with High-Static as a reference category with grey highlighting the significant independent variables

\begin{tabular}{|c|c|c|c|c|c|}
\hline IV Code & IV Description & Reference & Beta & Significance & Target \\
\hline INC.8 & & $\mathrm{H}-\mathrm{S}$ & 0.992 & 0.628 & L-S \\
\hline KSQ2F1 & & $\mathrm{H}-\mathrm{S}$ & 1.627 & 0.405 & H-D \\
\hline KSQ2F1 & & $\mathrm{H}-\mathrm{S}$ & 1.086 & 0.871 & L-I \\
\hline KSQ2F1 & & $\mathrm{H}-\mathrm{S}$ & 0.943 & 0.859 & $\mathrm{~L}-\mathrm{S}$ \\
\hline SUC.1 & $\begin{array}{l}\text { Success at solving } \\
\text { engineering problems: } \\
\text { "My ability or skill" }\end{array}$ & $\mathrm{H}-\mathrm{S}$ & 0.995 & 0.815 & L-S \\
\hline SUC.1 & & $\mathrm{H}-\mathrm{S}$ & 0.973 & 0.589 & H-D \\
\hline SUC.1 & & $\mathrm{H}-\mathrm{S}$ & 0.906 & 0.015 & L-I \\
\hline SUC.3 & $\begin{array}{l}\text { Success at solving } \\
\text { engineering problems: } \\
\text { "Told or requested to do } \\
\text { so by a teacher, boss, etc." }\end{array}$ & $\mathrm{H}-\mathrm{S}$ & 1.04 & 0.279 & L-I \\
\hline SUC.3 & & $\mathrm{H}-\mathrm{S}$ & 1.013 & 0.764 & H-D \\
\hline SUC.3 & & $\mathrm{H}-\mathrm{S}$ & 0.973 & 0.113 & L-S \\
\hline SUC.5 & $\begin{array}{l}\text { Success at solving } \\
\text { engineering problems: } \\
\text { "Support given to me by } \\
\text { my faculty, experts, or } \\
\text { team members" }\end{array}$ & H-S & 1.041 & 0.081 & L-S \\
\hline SUC.5 & & $\mathrm{H}-\mathrm{S}$ & 1.014 & 0.759 & H-D \\
\hline SUC.5 & & $\mathrm{H}-\mathrm{S}$ & 0.99 & 0.735 & L-I \\
\hline SUC.6 & $\begin{array}{l}\text { Success at solving } \\
\text { engineering problems: and } \\
\text { "The available resources } \\
\text { (i.e. materials or } \\
\text { facilities)" }\end{array}$ & $\mathrm{H}-\mathrm{S}$ & 1.023 & 0.723 & H-D \\
\hline SUC.6 & & $\mathrm{H}-\mathrm{S}$ & 0.976 & 0.495 & L-I \\
\hline SUC.6 & & $\mathrm{H}-\mathrm{S}$ & 0.942 & 0.031 & L-S \\
\hline
\end{tabular}


Table 11.3

Logistic regression with Low-Static as a reference category with grey highlighting the significant independent variables

\begin{tabular}{|c|c|c|c|c|c|}
\hline IV Code & IV Description & Reference & Beta & Significance & Target \\
\hline AF1_MAP & Mastery Approach & L-S & 2.151 & 0.031 & H-S \\
\hline AF1_MAP & & L-S & 1.147 & 0.787 & L-I \\
\hline AF1_MAP & & $\mathrm{L}-\mathrm{S}$ & 0.615 & 0.478 & H-D \\
\hline EAM & Anxiety Mean & L-S & 1.051 & 0.072 & H-D \\
\hline EAM & & $\mathrm{L}-\mathrm{S}$ & 1.012 & 0.444 & $\mathrm{H}-\mathrm{S}$ \\
\hline EAM & & $\mathrm{L}-\mathrm{S}$ & 0.987 & 0.591 & L-I \\
\hline ESM & Success Mean & L-S & 1.072 & 0.005 & H-S \\
\hline ESM & & L-S & 1.007 & 0.832 & H-D \\
\hline ESM & & L-S & 0.957 & 0.063 & L-I \\
\hline FQ1.2 & $\begin{array}{l}\text { How often did you feel... } \\
\text { "That you have } \\
\text { experiences that } \\
\text { challenge you to grow or } \\
\text { become a better person?" }\end{array}$ & L-S & 1.578 & 0.267 & $\mathrm{H}-\mathrm{S}$ \\
\hline FQ1.2 & & L-S & 1.57 & 0.457 & H-D \\
\hline FQ1.2 & & $\mathrm{L}-\mathrm{S}$ & 0.802 & 0.692 & L-I \\
\hline FQ1.4 & $\begin{array}{l}\text { How often did you feel... } \\
\text { "Confident to think or } \\
\text { express your own ideas } \\
\text { and opinions?" }\end{array}$ & $\mathrm{L}-\mathrm{S}$ & 2.421 & 0.338 & H-D \\
\hline FQ1.4 & & $\mathrm{L}-\mathrm{S}$ & 1.088 & 0.905 & L-I \\
\hline FQ1.4 & & L-S & 0.96 & 0.948 & $\mathrm{H}-\mathrm{S}$ \\
\hline FQ1F & $\begin{array}{l}\text { Factor } 1 \text { from factor } \\
\text { analysis for "In the last } \\
\text { month how often did you } \\
\text { feel..." }\end{array}$ & $\mathrm{L}-\mathrm{S}$ & 0.831 & 0.842 & L-I \\
\hline FQ1F & & $\mathrm{L}-\mathrm{S}$ & 0.826 & 0.798 & $\mathrm{H}-\mathrm{S}$ \\
\hline FQ1F & & $\mathrm{L}-\mathrm{S}$ & 0.445 & 0.43 & H-D \\
\hline INC. 2 & $\begin{array}{l}\text { Incentive to participate: } \\
\text { "Help society or solve a } \\
\text { societal need" }\end{array}$ & L-S & 1.056 & 0.029 & H-S \\
\hline INC.2 & & L-S & 1.006 & 0.832 & L-I \\
\hline INC.2 & & L-S & 0.984 & 0.663 & H-D \\
\hline
\end{tabular}


Table 11.4

Continuation of Logistic regression with Low-Static as a reference category with grey highlighting the significant independent variables

\begin{tabular}{|c|c|c|c|c|c|}
\hline IV Code & IV Description & Reference & Beta & Significance & Target \\
\hline INC.4 & $\begin{array}{l}\text { Incentive to participate: } \\
\text { "Learn something new and/or } \\
\text { gain experience" }\end{array}$ & L-S & 0.999 & 0.976 & $\mathrm{H}-\mathrm{S}$ \\
\hline INC.4 & & L-S & 0.98 & 0.692 & H-D \\
\hline$\overline{\mathrm{INC} .4}$ & & L-S & 0.977 & 0.506 & L-I \\
\hline INC.7 & $\begin{array}{l}\text { Incentive to participate: } \\
\text { "Recognition" }\end{array}$ & L-S & 1.029 & 0.449 & H-D \\
\hline INC.7 & & $\mathrm{L}-\mathrm{S}$ & 0.997 & 0.922 & L-I \\
\hline INC.7 & & L-S & 0.954 & 0.034 & $\mathrm{H}-\mathrm{S}$ \\
\hline INC. 8 & $\begin{array}{l}\text { Incentive to participate: } \\
\text { "Presents a challenge" }\end{array}$ & L-S & 1.039 & 0.104 & $\mathrm{H}-\mathrm{S}$ \\
\hline INC. 8 & & L-S & 1.006 & 0.899 & H-D \\
\hline INC. 8 & & L-S & 0.999 & 0.966 & L-I \\
\hline$\overline{\text { KSQ1F1 }}$ & Personal Strengths & L-S & 2.991 & 0.035 & L-I \\
\hline KSQ1F1 & & L-S & 0.934 & 0.832 & H-S \\
\hline KSQ1F1 & & L-S & 0.9 & 0.848 & H-D \\
\hline KSQ1F4 & Future Plans & L-S & 2.224 & 0.023 & H-S \\
\hline KSQ1F4 & & L-S & 1.291 & 0.648 & H-D \\
\hline KSQ1F4 & & L-S & 1.126 & 0.788 & L-I \\
\hline SUC.1 & $\begin{array}{l}\text { Success at solving } \\
\text { engineering problems: "My } \\
\text { ability or skill" }\end{array}$ & L-S & 1.035 & 0.182 & $\mathrm{H}-\mathrm{S}$ \\
\hline SUC.1 & & L-S & 0.974 & 0.609 & H-D \\
\hline SUC.1 & & L-S & 0.915 & 0.059 & L-I \\
\hline SUC.3 & $\begin{array}{l}\text { Success at solving } \\
\text { engineering problems: "Told } \\
\text { or requested to do so by a } \\
\text { teacher, boss, etc." }\end{array}$ & L-S & 1.117 & 0.011 & L-I \\
\hline SUC.3 & & L-S & 1.01 & 0.828 & H-D \\
\hline SUC.3 & & L-S & 1 & 0.993 & $\mathrm{H}-\mathrm{S}$ \\
\hline SUC.5 & $\begin{array}{l}\text { Success at solving } \\
\text { engineering problems: } \\
\text { "Support given to me by my } \\
\text { faculty, experts, or team } \\
\text { members" }\end{array}$ & L-S & 0.987 & 0.841 & H-D \\
\hline SUC.5 & & L-S & 0.978 & 0.461 & L-I \\
\hline SUC.5 & & L-S & 0.955 & 0.068 & H-S \\
\hline
\end{tabular}


Table 11.5

Continuation of Logistic regression with Low-Static as a reference category with grey highlighting the significant independent variables

\begin{tabular}{|c|l|c|c|c|c|}
\hline IV Code & \multicolumn{1}{|c|}{ IV Description } & Reference & Beta & Significance & Target \\
\hline SUC.6 & $\begin{array}{l}\text { Success at solving } \\
\text { engineering problems: } \\
\text { and "The available } \\
\text { resources (i.e. } \\
\text { materials or facilities)" }\end{array}$ & L-S & $\mathbf{1 . 1 4 2}$ & $\mathbf{0 . 0 9 3}$ & H-D \\
\hline SUC.6 & & L-S & $\mathbf{1 . 0 5 1}$ & $\mathbf{0 . 0 9 2}$ & H-S \\
\hline SUC.6 & & L-S & 1.015 & 0.696 & L-I \\
\hline
\end{tabular}




\section{Appendix: ISES Interview Codes}

Table 12.1

ISES interview codes and definitions

\begin{tabular}{|l|l|}
\hline Code Name & Definition \\
\hline Achievement & $\begin{array}{l}\text { Accomplishing something related to engineering } \\
\text { design }\end{array}$ \\
\hline $\begin{array}{l}\text { Application of Knowledge } \\
\text { and Skills }\end{array}$ & Utilizing engineering coursework and experiences \\
\hline Autonomy & The desire to do things independently \\
\hline Avoidance & $\begin{array}{l}\text { Not wanting to do a specific task related to } \\
\text { engineering, procrastination with a task }\end{array}$ \\
\hline Challenge & $\begin{array}{l}\text { When engineering tasks require students to put more } \\
\text { effort into them }\end{array}$ \\
\hline Confidence & Feeling able to complete a task \\
\hline Coursework & $\begin{array}{l}\text { Specific courses leading to a deeper interest to } \\
\text { continue pursuing engineering }\end{array}$ \\
\hline Creativity & $\begin{array}{l}\text { Being able to utilize skills to solve problems in } \\
\text { unique manners }\end{array}$ \\
\hline Curiosity & $\begin{array}{l}\text { Interest and intrigue for the engineering field, task, or } \\
\text { experience }\end{array}$ \\
\hline Environment & $\begin{array}{l}\text { The surrounding atmosphere encouraging students to } \\
\text { continue working with engineering skills }\end{array}$ \\
\hline Family & $\begin{array}{l}\text { Support from family as well as family members } \\
\text { working in the same field encourage students }\end{array}$ \\
\hline Friends & Support from peers and close friends to continue \\
\hline Future Circumstances & Keeping later goals in mind \\
\hline Helping Others & $\begin{array}{l}\text { The possibility of making a difference for someone } \\
\text { else }\end{array}$ \\
\hline Interest/Enjoyment & Personal fulfillment in doing engineering work \\
\hline Knowledge & Learning something new \\
\hline Mastery & Learning engineering tasks/skills better than others \\
\hline Mention of Motivation & Students commenting that they are motivated \\
\hline Mentor & $\begin{array}{l}\text { An advisor, teacher, or friend promoting engineering } \\
\text { for the student }\end{array}$ \\
\hline Mindset & $\begin{array}{l}\text { Mentality leading the student to want to do } \\
\text { engineering }\end{array}$ \\
\hline New Experience & $\begin{array}{l}\text { The prospect of a new experience motivating the } \\
\text { student to do engineering design work }\end{array}$ \\
\hline New Perspective & Finding a new way to look at things \\
\hline &
\end{tabular}


Table 12.2

Continuation of ISES interview codes and definitions

\begin{tabular}{|c|c|}
\hline Code Name & Definition \\
\hline Occupational Choice & $\begin{array}{l}\text { Wanting to make a career out of } \\
\text { engineering }\end{array}$ \\
\hline Performance & $\begin{array}{l}\text { Feeling prepared and doing well on a } \\
\text { specific task }\end{array}$ \\
\hline Persistence & The drive to continue with engineering \\
\hline Personal Significance & $\begin{array}{l}\text { Specific aspects of engineering } \\
\text { experiences providing internal } \\
\text { significance }\end{array}$ \\
\hline Physiological State & $\begin{array}{l}\text { Commenting on how an experience } \\
\text { made them feel }\end{array}$ \\
\hline Real World Experience & $\begin{array}{l}\text { Utilizing engineering skills and } \\
\text { knowledge in a way they will be used in } \\
\text { a paid engineering position }\end{array}$ \\
\hline Reward & $\begin{array}{l}\text { A feeling or gift (i.e. grade) received for } \\
\text { an engineering related service }\end{array}$ \\
\hline Social Persuasion & $\begin{array}{l}\text { Peer pressure to continue working in the } \\
\text { field }\end{array}$ \\
\hline Task-Involved & Motivation related to performing a task \\
\hline Travel & $\begin{array}{l}\text { The opportunity to travel to doing } \\
\text { engineering design work }\end{array}$ \\
\hline Work Avoidance & $\begin{array}{l}\text { Not wanting to work causing students to } \\
\text { procrastinate or avoid a specific task }\end{array}$ \\
\hline
\end{tabular}




\section{Appendix: Copyright Permission Form Documentation for Portions of Chapters 4 and 5}

Paper procedures using Monolith

\section{ASEE ANNUAL CONFERENCE \& EXPOSITION COPYRIGHT TRANSFER FORM}

Title of Paper:

Author(s):

Publication: ASEE Annual Conference Proceedings $\quad$ Session \#:

PART A

The undersigned, desiring to publish the above paper in a publication of ASEE or co-sponsored by ASEE, hereby transfers their copyrights in the above paper to the American Society for Engineering Education, known as ASEE.

In return for these rights, ASEE hereby grants the above author(s), and the employers for whom the work was performed, permission to:

-- Reuse portions of the above paper in other works.

-- Reproduce the above paper for personal or internal use, provided that (a) the source and ASEE copyright are indicated, (b) the copies are not used in a way that implies ASEE endorsement of product or service of an employer, and (c) the copies are not offered for sale.

In exercising its rights under copyright, ASEE will make all reasonable efforts to act in the interests of the authors or their employers as well as in its own interest. In particular, ASEE requires that:

-- The consent of the first-named author is sought as a condition in granting republication permission by others.

-- The consent of the authors or their employers be obtained as a condition in granting permission to others to reuse a portion or all of the paper for promotion or marketing purposes.

Name:

Signature:

Employer:
Title: (if not author, then relationship to author) Date Form Signed:

\section{PART B}

(For U.S. Government Employees Only)

This will certify that all authors of the above paper are employees of the U.S. Government and performed this work as part of their employment.

Name:

Signature:

Govt. Organization:
Title: (if not author, then relationship to author) Date Form Signed:

Copyright Transfers may be sent via fax or mail using the following:

ASEE - Convention \& Seminar Corporation

1818 N Street NW, Suite 600

Washington, DC 20036

ATTN: Copyright Transfer Form

Fax: (202) 265-8504

Questions? Call (202) 649-3829 